\title{
Araçá: biodiversidade, impactos e ameaças
}

\author{
Antonia Cecília Zacagnini Amaral ${ }^{1,4}$, Alvaro Esteves Migotto ${ }^{2}$, \\ Alexander Turra ${ }^{3}$ \& Yara Schaeffer-Novelli ${ }^{3}$ \\ ${ }^{1}$ Departamento de Biologia Animal, Instituto de Biologia, Universidade Estadual de Campinas - UNICAMP, \\ CP 6109, CEP 13083-970 Campinas, SP, Brasil \\ ${ }^{2}$ Centro de Biologia Marinha, Universidade de São Paulo - USP, \\ Rodovia Manuel Hipólito do Rego, Km 131,5, CEP 11600-000 \\ São Sebastião, SP, Brasil, e-mail: aemigott@usp.br \\ ${ }^{3}$ Departamento de Oceanografia Biológica, Instituto Oceanográfico, Universidade de São Paulo - USP, \\ Praça do Oceanográfico, 191, Cidade Universitária, CEP 05508-120 São Paulo, SP, Brasil, \\ e-mail: turra@usp.br;novelliy@usp.br \\ ${ }^{4}$ Autor para correspondência: Antonia Cecília Zacagnini Amaral, e-mail: ceamaral@unicamp.br
}

AMARAL, A.C.Z., MIGOTTO, A.E., TURRA, A. \& SCHAEFFER-NOVELLI, Y. Araçá: biodiversity, impacts and threats. Biota Neotrop.,10(1): http://www.biotaneotropica.org.br/v10n1/en/abstract?inventory+bn01210012010.

Abstract:Araçá Bay (São Sebastião, State of São Paulo), within one of Brazil's most beautiful coastlines, has small relict mangrove stands and a very diverse marine ecosystem. As such, the bay is a natural laboratory as well as important for local small scale fishing. In this study we summarize the large literature base to comprehensibly list the flora and fauna as a preliminary biodiversity inventory of Araçá Bay. We place this in the historical context of human impact on the environment of the bay and we emphasize new, introduced and threatened species as bioindicators and natural resources. With this information, we provide a basis to inform conservation decisions as well as data for conservation management plans and call attention to the urgent need to protect this fragile environment and biota. We also emphasize that this small and uniquely biologically rich bay should be preserved, revitalized and integrated into the growing urban environment.

Keywords: flora, fauna, alterations, threats, conservation, Araçá.

AMARAL, A.C.Z., MIGOTTO, A.E., TURRA, A. \& SCHAEFFER-NOVELLI, Y. Araçá: biodiversidade, impactos e ameaças. Biota Neotrop. 10(1): http://www.biotaneotropica.org.br/v10n1/pt/abstract?inventory+bn01210012010.

Resumo: Inserida em uma das mais belas e turísticas regiões litorâneas do país - o Litoral Norte do Estado de São Paulo -, a Baía do Araçá (São Sebastião, SP), além de conter remanescentes de manguezal e de abrigar alta diversidade biológica, é também um verdadeiro laboratório a céu aberto e um importante reduto de catadores de moluscos e pescadores artesanais. Com o objetivo de compilar preliminarmente a biodiversidade da baía, este estudo reúne a extensa bibliografia dedicada à região e apresenta a lista das espécies da fauna e da flora formalmente reportadas para o local. O texto sumaria ainda o histórico das alterações antrópicas sofridas nas últimas décadas e destaca certos representantes da fauna (espécies novas, ameaçadas de extinção, bioindicadoras e como recursos naturais), como exemplos da peculiaridade e riqueza biológica dessa pequena baía. Pretende-se, assim, fornecer subsídios para tomadas de decisão e planos de manejo, e despertar a atenção para a preservação desse frágil patrimônio ambiental, uma área que pode ser revitalizada e integrada ao ambiente urbanizado.

Palavras-chave: flora, fauna, alterações, ameaças, conservação, Araçá. 


\section{Porque Conservar o Araçá para as Futuras Gerações?}

No início de um novo milênio a sociedade brasileira enfrenta grandes desafios na busca de um estilo de vida capaz de conciliar desenvolvimento com conservação ambiental (CIMA 1991). Essa mudança de paradigma vem sendo buscada há décadas e requer alterações culturais profundas na sociedade, incluindo princípios éticos como os da precaução, prevenção e equidade entre gerações. Contemplando esse conceito de sustentabilidade, esta pesquisa tem como objetivo relatar a importância da Baía do Araçá, São Sebastião (SP), do ponto de vista biológico, e apresentar alternativas à intervenção do homem nos processos de funcionamento desse ambiente para, inclusive, estimular iniciativas que revertam o quadro atual de degradação ambiental.

A Baía do Araçá (Figura 1a-b) mantém um dos últimos remanescentes de manguezal do litoral de São Sebastião. De acordo com Lamparelli et al. (1998), devido à proximidade da Serra do Mar, que limita em extensão as planícies costeiras, o Litoral Norte do Estado de São Paulo possui poucas áreas de manguezais $\left(3,4 \mathrm{~km}^{2}\right)$, sendo que apenas $1 \%$ encontra-se no Município de São Sebastião, restritas principalmente ao interior de baías. O Araçá, além de conter remanescentes de manguezal e de abrigar alta diversidade biológica, é também importante reduto de pescadores artesanais que utilizam pequenas canoas caiçaras, uma tradição do Litoral Norte, para capturar peixes e crustáceos.

Os manguezais são considerados ecossistemas vitais para vida marinha. É nesse ambiente que diversas espécies se reproduzem, se alimentam e buscam abrigo e proteção. Apesar da aparência desorganizada quando observados durante a maré baixa, devido à quantidade de raízes, troncos e pneumatóforos sobre o sedimento, esse ecossistema é altamente estruturado. O manguezal, segundo definição de Schaeffer-Novelli (1991), constitui um "Ecossistema costeiro, de transição entre os ambientes terrestre e marinho, característico de regiões tropicais e subtropicais, sujeito ao regime de marés. É constituído de espécies vegetais e macroalgas (criptógamas), adaptadas à flutuação de salinidade e caracterizados por colonizarem sedimentos predominantemente lodosos, com baixos teores de oxigênio. Ocorre em regiões costeiras abrigadas e apresenta condições propícias para alimentação, proteção e reprodução de muitas espécies animais, sendo considerado importante transformador de nutrientes em matéria orgânica e gerador de bens e serviços".

Apesar dessa importância, os manguezais encontram-se sujeitos às mais variadas formas de intervenção antrópica, das quais se destacam: extrativismo, dragagens, aterros, lixões, construção de portos, empreendimentos imobiliários, agricultura e poluição industrial e urbana. Em antagonismo, tem-se a relação direta entre comunidades tradicionais litorâneas e os manguezais. É notório, como já observado por Diegues (1987), que estas comunidades desenvolveram um modo de vida em que as atividades econômicas, sociais e culturais dependem fundamentalmente, direta e indiretamente, desses ecossistemas e dos ciclos biológicos que neles se desenvolvem.

\section{A Baía do Araçá}

O Araçá é uma pequena enseada limitada por flancos rochosos e que abrange quatro praias (Deodato, Pernambuco, Germano e Topo), duas ilhotas (Pernambuco e Pedroso), três núcleos principais de bosques de manguezal, e uma extensa planície de fundo mole, descoberta inteiramente em períodos de maré baixa de sizígia (Figura 1a-b). Essa configuração impede que a região seja submetida diretamente aos fatores hidrodinâmicos procedentes de fora do Canal de São Sebastião. A fisionomia resultante é composta por praias de declive suave, com largura da zona entremarés de 50-300 m, constituídas por sedimentos areno-lamosos, relativamente compactos (Figura 2a-b), e cascalhos. Assim, o Araçá pode ser classificado como uma baía muito protegida, com hidrodinâmica dominada pela maré, mas que também sofre a ação de ondas de baixa amplitude, e vegetação e fauna características de manguezal (Figuras 3-8). Devido à proximidade da malha urbana, esse conjunto de pequenas praias, costões rochosos (Figuras 9-11), bancos arenosos e lamosos (Figuras 12-18), ilhotes e núcleos de manguezal está, há muitos anos, exposto a diferentes tipos de ação antrópica, como ocupações irregulares, efluentes de esgoto doméstico e proximidade do Porto de São Sebastião e o Terminal Aquaviário da Petrobras, onde ocorrem constantes vazamentos de óleos, além de outras perturbações.

\section{Histórico de Alterações e Sobrevivência do Araçá}

Os manguezais do litoral brasileiro têm chamado a atenção de estudiosos e naturalistas desde o período colonial, resultando em uma volumosa literatura versando sobre variados aspectos desses ecossistemas (Schaeffer-Novelli 1986). Entre os primeiros estudos científicos realizados no Brasil, destaca-se o de Luederwaldt (1919), sobre os manguezais de Santos. Até a década de 1970, a maioria dos estudos tinha caráter descritivo da fauna e, sobretudo, da flora e dos usos tradicionais de seus recursos naturais pela população caiçara. Já naquela década, os cientistas de diferentes partes do mundo estavam impressionados pelo estado de degradação dos manguezais (Vannucci 2002). A partir de 1980, programas de pesquisas interdisciplinares passaram a abordar os manguezais em nível de ecossistema.

A Baía do Araçá vem sofrendo alterações em suas características originais principalmente em consequiência de inúmeras obras realizadas a partir de 1936 e intensificadas nas décadas de 1970 e 1980. Antes da construção do Porto de São Sebastião, iniciada em 1936 e finalizada apenas em 1955, as praias que hoje formam o Araçá eram praticamente contínuas com a Praia de São Sebastião, localizada em frente à Rua da Praia. Além de isolar o Araçá da orla central da cidade, a barreira criada pelo cais do porto alterou a circulação natural das águas, iniciando ou agravando o processo de assoreamento na praia da frente da cidade. A expansão da área portuária, por meio de aterros e de enrocamentos, acelerou o assoreamento do seu entorno, sobretudo na parte interna do cais, fazendo com que a praia da cidade fosse desaparecendo gradativamente. Aterros executados em ambos os lados do cais, nas décadas de 1970 e 1980, retalharam parte da baía, eliminando de vez o que restou da praia em frente do centro histórico de São Sebastião (Francisco \& Carvalho 2003).

Embora a idéia de se aterrar o Araçá para fins portuários seja muito antiga (CGG 1919), esforços nesse sentido ocorreram de fato em meados da década de 1980, quando então o Departamento Hidroviário da Secretaria de Transportes do Estado de São Paulo pretendia expandir a área portuária e do retroporto, prevendo o aumento da movimentação do caís público. O projeto não foi aprovado pela Secretaria do Meio Ambiente, mormente devido a pressão de ambientalistas e da comunidade científica. Nesse mesmo período, o Araçá foi seccionado diagonalmente por uma desastrosa dragagem (cerca de $6 \mathrm{~m}$ de profundidade) para instalação de um emissário submarino de esgoto sanitário da Sabesp (Companhia de Saneamento Básico do Estado de São Paulo), a partir da região superior da Praia do Germano até o limite entre a zona inferior das marés e início do infralitoral, no extremo sul da baía. O sedimento ressuspendido, composto, principalmente, de areia média, grossa e cascalho, depositou-se nas proximidades da Ponta do Araçá (lado sudoeste), formando um banco de aproximadamente $2 \mathrm{~m}$ de altura, onde o teor de carbonato de cálcio atingiu $35,70 \%$. No restante da baía as areias fina e muito fina, com significativas parcelas de silte, foram recobertas por uma camada espessa de coloração cinza-esverdeada e de aspecto coloidal, tornando-se uma 

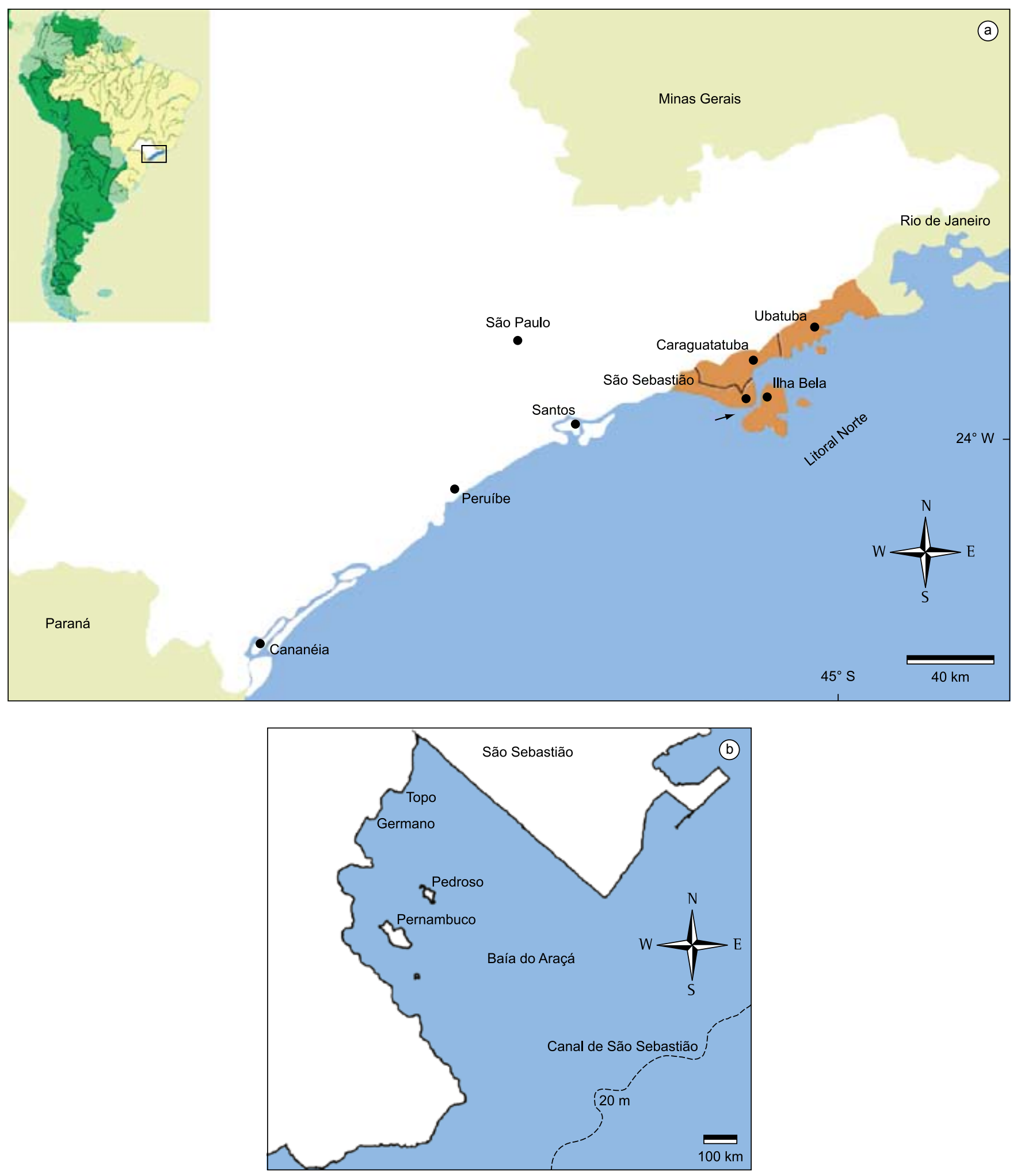

Figura 1. a) Estado de São Paulo e detalhe do Litoral Norte e Canal de São Sebastião (seta) (adaptado de Lamparelli et al. 1998); b) Baia do Araçá (adaptado de Amaral \& Morgado 1994).

Figure 1. a) São Paulo State and detail of the Northern Coast with São Sebastião Channel (arrow) (modified from Lamparelli et al. 1998); b) Araçá Bay (modified from Amaral \& Morgado 1994). 

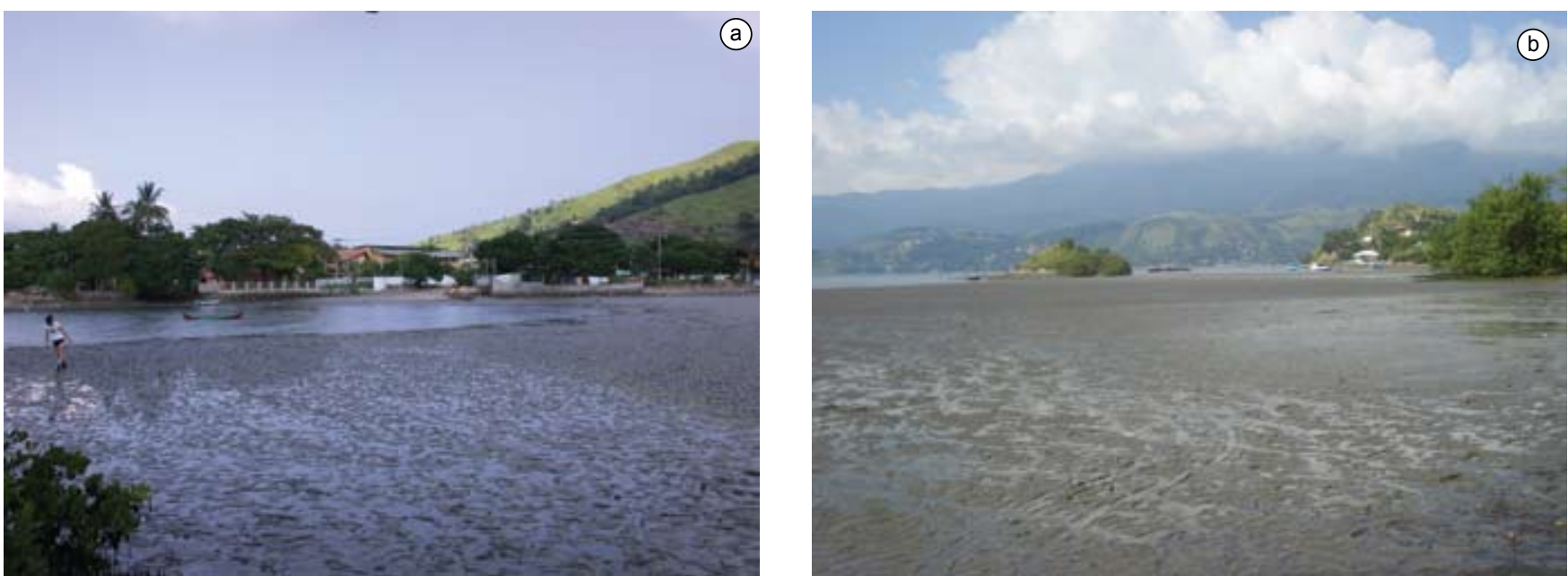

Figura 2. a-b) Baía do Araçá: região entremarés, fundo areno-lamoso. Fotos: a) S.A. Nallin (05/2009); b) A.C.Z. Amaral (07/2009).

Figure 2. a-b) Araçá Bay: intertidal zone, muddy sand flat. Photos: a) S.A. Nallin (05/2009); b) A.C.Z. Amaral (07/2009).

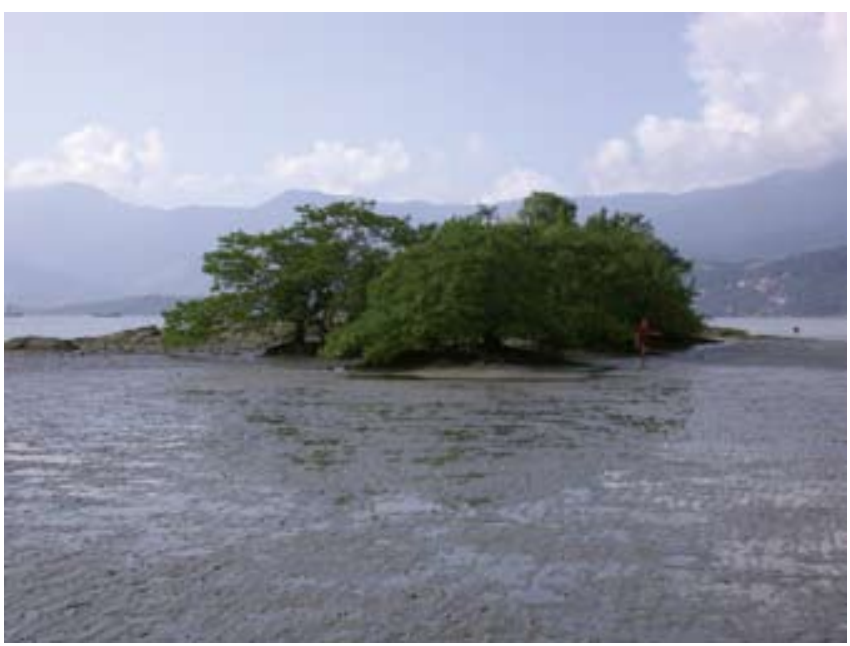

Figura 3. Baía do Araçá: vista geral do núcleo de manguezal localizado nas proximidades da Ilha Pernambuco. Foto: S.A. Nallin (05/2009).

Figure 3. Araçá Bay: general view of mangrove vegetation located near Pernambuco Island. Photo: S.A. Nallin (05/2009).

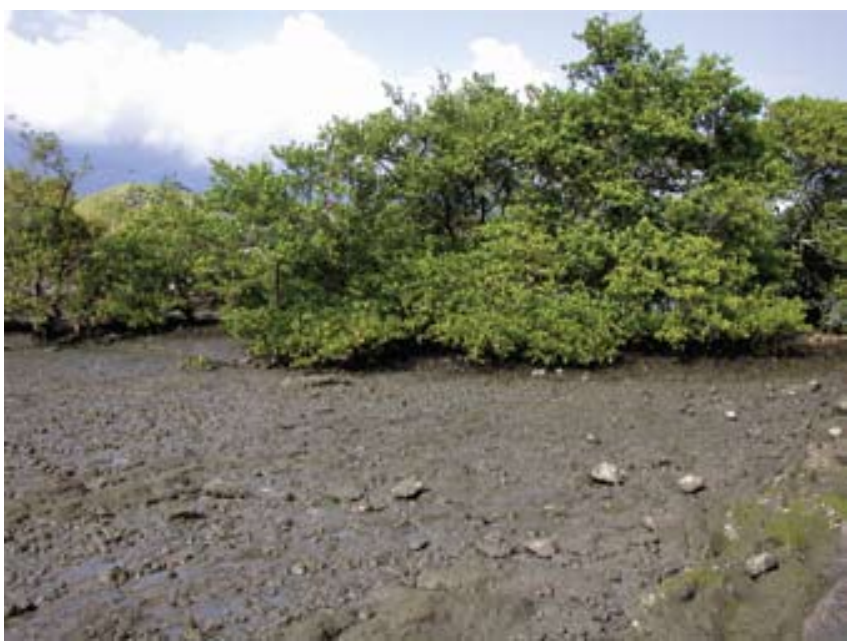

Figura 4. Baía do Araçá: vegetação do núcleo de manguezal localizado nas proximidades da Ilha Pernambuco. Foto: S.A. Nallin (05/2009).

Figure 4. Araçá Bay: mangrove vegetation located near Pernambuco Island. Photo: S.A. Nallin (05/2009).

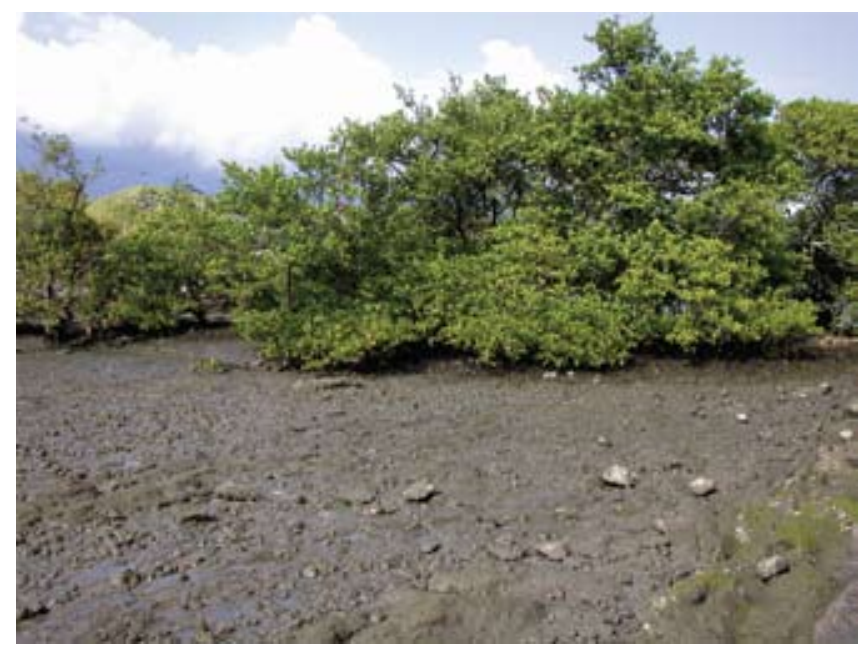

Figura 5. Baía do Araçá: interior do núcleo de manguezal localizado nas proximidades da Ilha Pernambuco. Foto: S.A. Nallin (05/2009).

Figure 5. Araçá Bay: view of the interior of the mangrove located near Pernambuco Island. Photo: S.A. Nallin (05/2009).

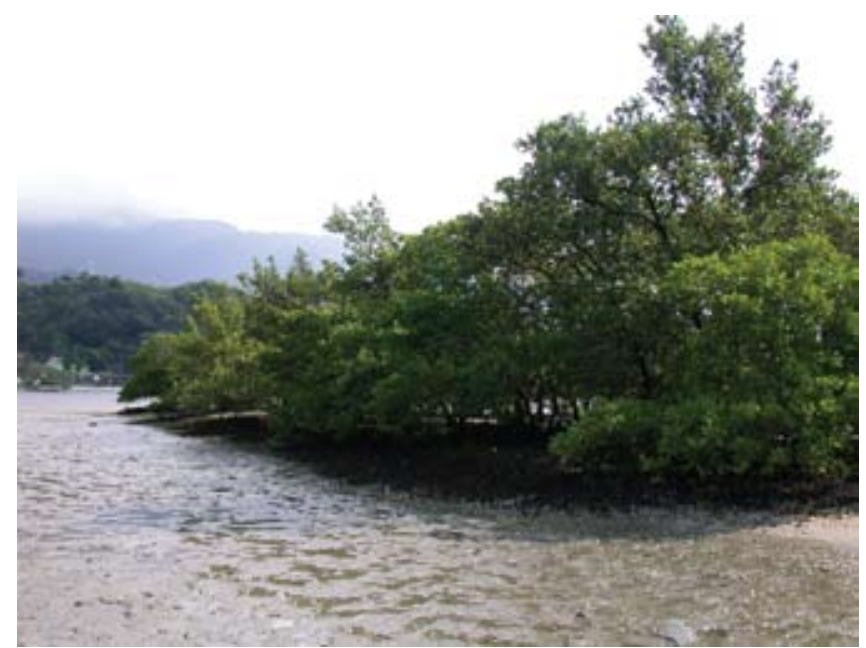

Figura 6. Baía do Araçá: núcleo de manguezal localizado na parte superior da região entremarés. Foto: S.A. Nallin (05/2009).

Figure 6. Araçá Bay: mangrove vegetation, high intertidal zone. Photo: S.A. Nallin (05/2009). 


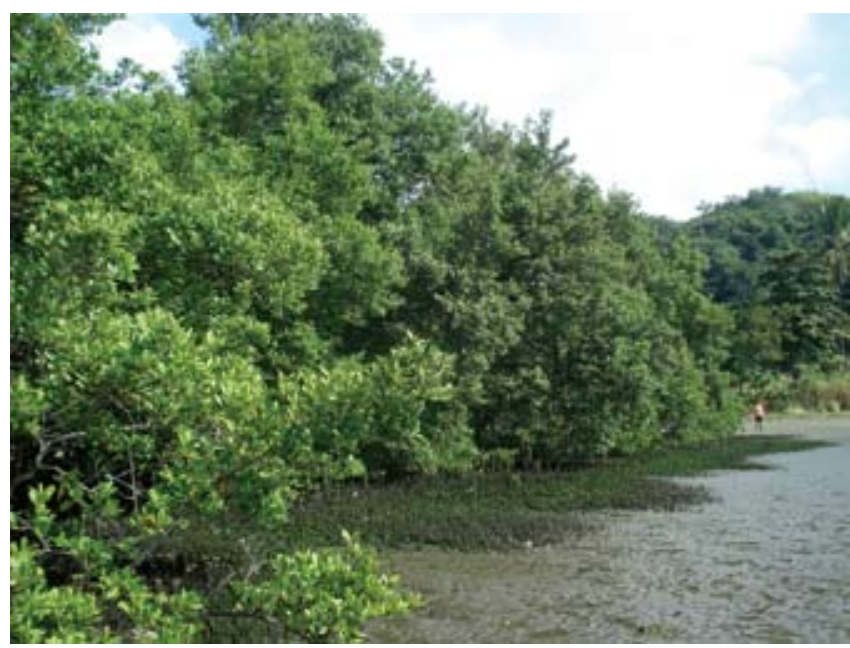

Figura 7. Baía do Araçá: vegetação do núcleo de manguezal localizado na parte superior da região entremarés. Foto: A.C.Z. Amaral (07/2009).

Figure 7. Araçá Bay: mangrove vegetation, high intertidal zone. Photo: A.C.Z. Amaral (07/2009).

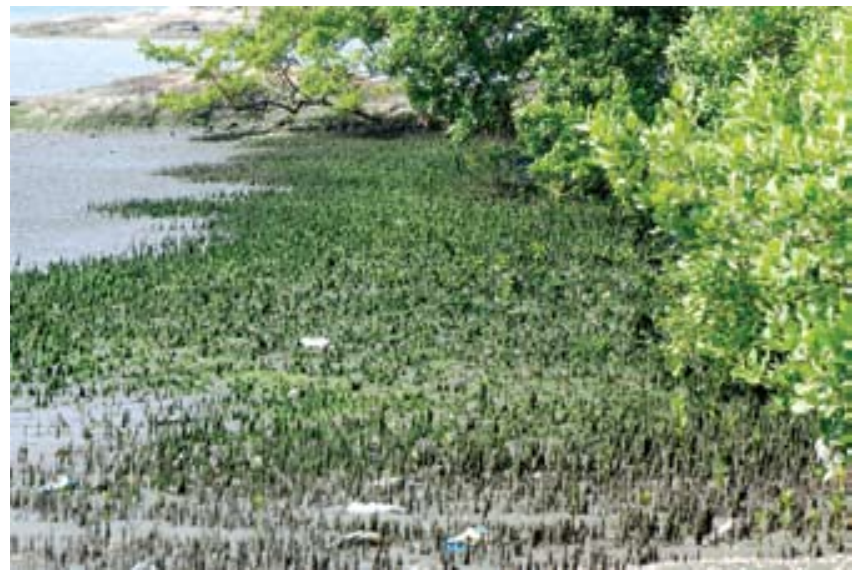

Figura 8. Baía do Araçá: detalhe do núcleo de manguezal localizado na parte superior da região entremarés. Foto A.E. Migotto (07/2009)

Figure 8. Araçá Bay: detail of the mangrove vegetation, high intertidal zone. Photo A.E. Migotto (07/2009).

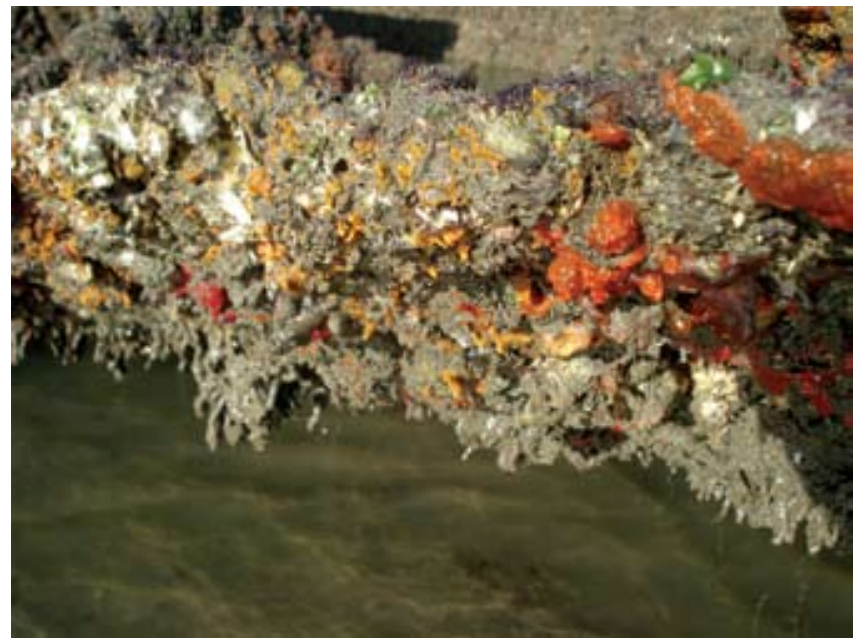

Figura 9. Baía do Araçá: costão rochoso da Ilha Pernambuco, zona inferior da região entremarés. Foto: A.C.Z. Amaral (07/2009).

Figure 9. Araçá Bay: low intertidal rocky shore, Pernambuco Island. Photo: A.C.Z. Amaral (07/2009).

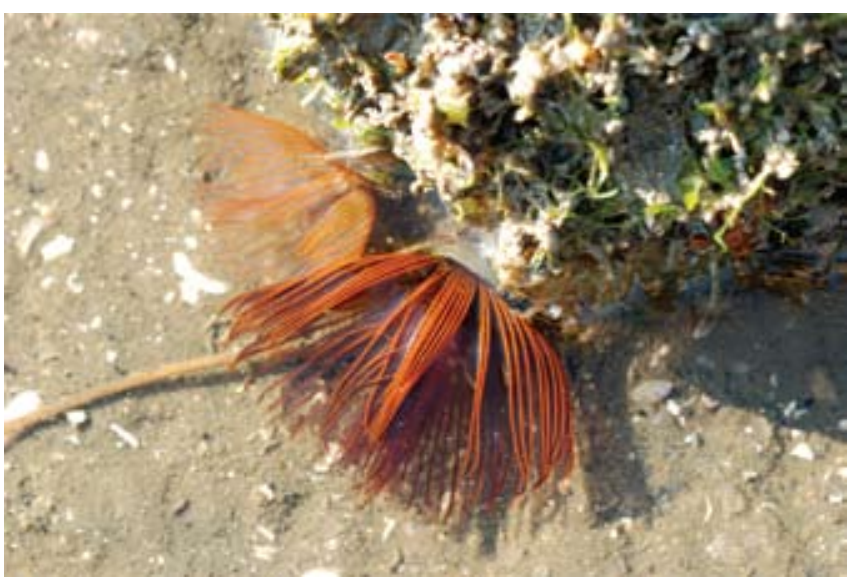

Figura 10. Baía do Araçá: costão rochoso da Ilha Pernambuco, em evidência poliquetas sabelídeos Branchiomma luctuosum. Foto: A.E. Migotto (07/2009).

Figure 10. Araçá Bay: rocky shore of Pernambuco Island, showing Sabellid polychaetes Branchiomma luctuosum. Photo: A.E. Migotto (07/2009).

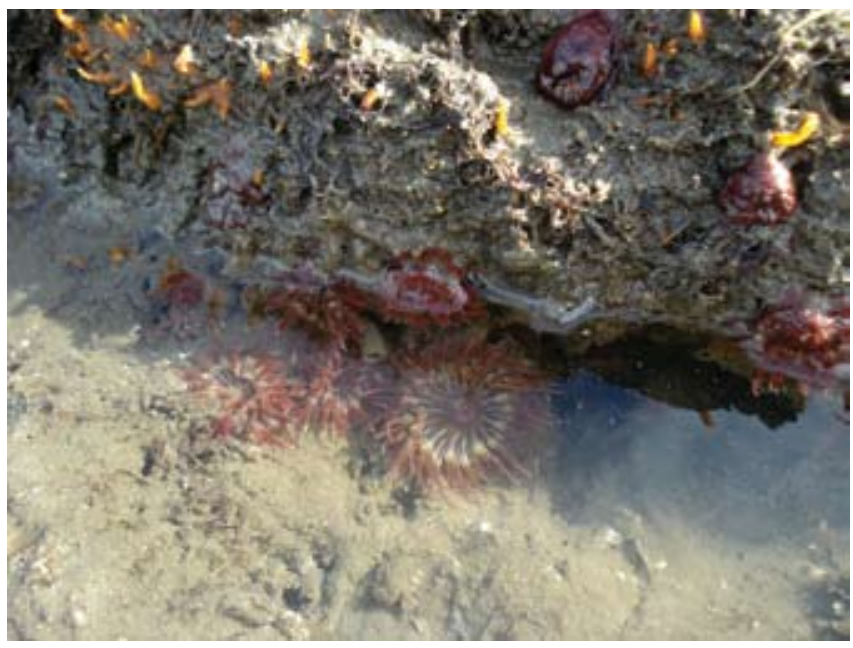

Figura 11. Baía do Araçá: costão rochoso da Ilha Pernambuco, em evidência anêmonas-do-mar Anemonia sargassensis. Foto: A.C.Z. Amaral (07/2009).

Figure 11. Araçá Bay: rocky shore of Pernambuco Island, showing sea anemones Anemonia sargassensis. Photo: A.C.Z. Amaral (07/2009).

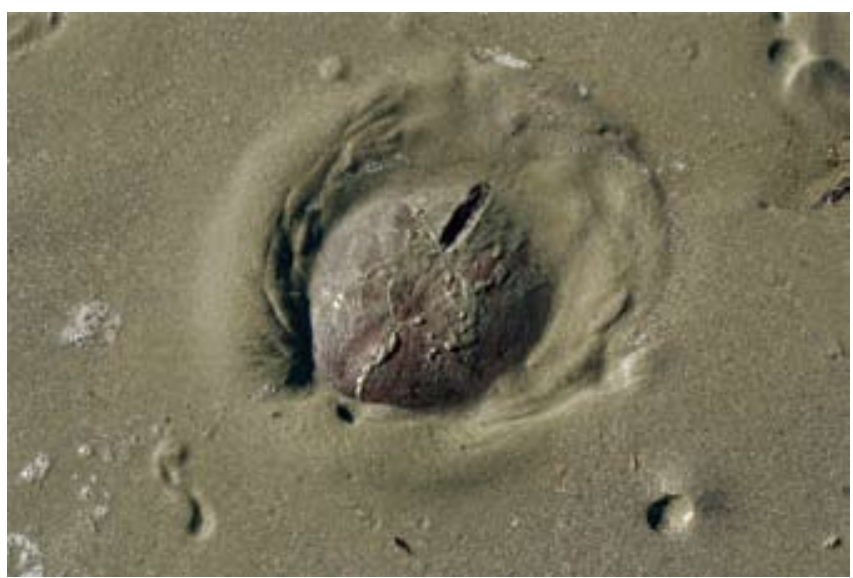

Figura 12. Baía do Araçá: bolacha-do-mar Encope emarginata em sedimento areno-lamoso, zona inferior da região entremarés. Foto: A.E. Migotto (07/2009).

Figure 12. Araçá Bay: the sand dollar Encope emarginata in muddy sand flat, low intertidal zone. Photo: A.E. Migotto (07/2009). 


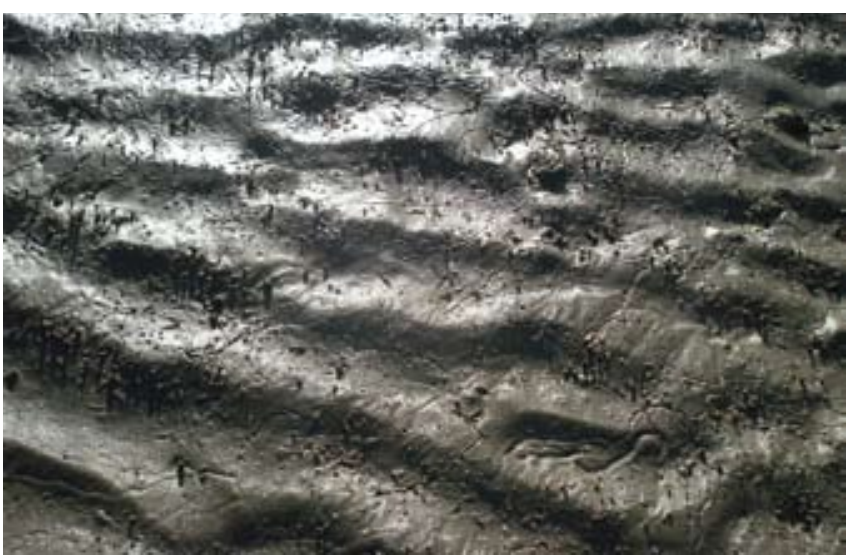

Figura 13. Baía do Araçá: pequenos tubos do crustáceo Kalliapseudes e marcas do gastrópode Olivella minuta em sedimento areno-lamoso na região entremarés. Foto: A.C.Z. Amaral (07/2009).

Figure 13. Araçá Bay: small tubes of the crustacean Kalliapseudes, and sinuous trail marks of the gastropod Olivella minuta in muddy sand flat, intertidal zone. Photo: A.C.Z. Amaral (07/2009).

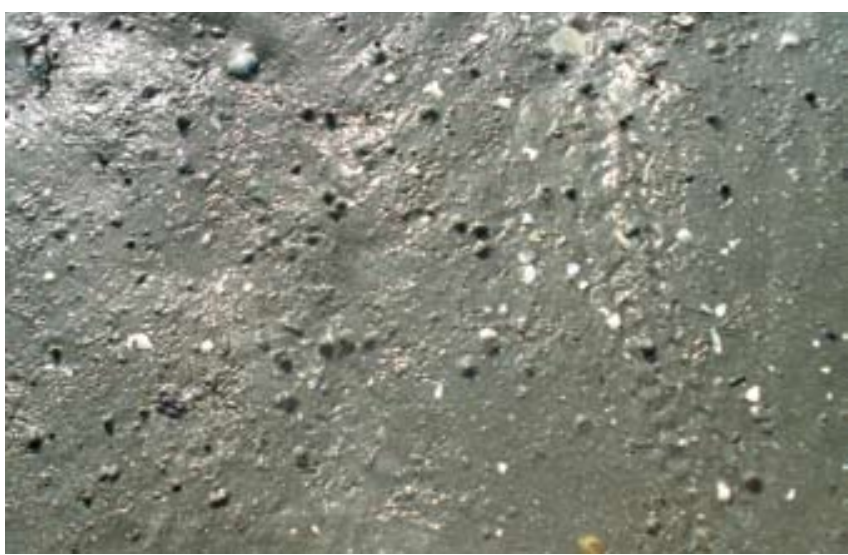

Figura 14. Baía do Araçá: dejetos na superfície do sedimento areno-lamoso que caracteriza a presença do poliqueta Laeonereis culveri. Foto: A.C.Z. Amaral (07/2009).

Figure 14. Araçá Bay: aggregates of fecal material on the surface of muddy sand bed produced by the polychaete Laeonereis culveri. Photo: A.C.Z. Amaral (07/2009).

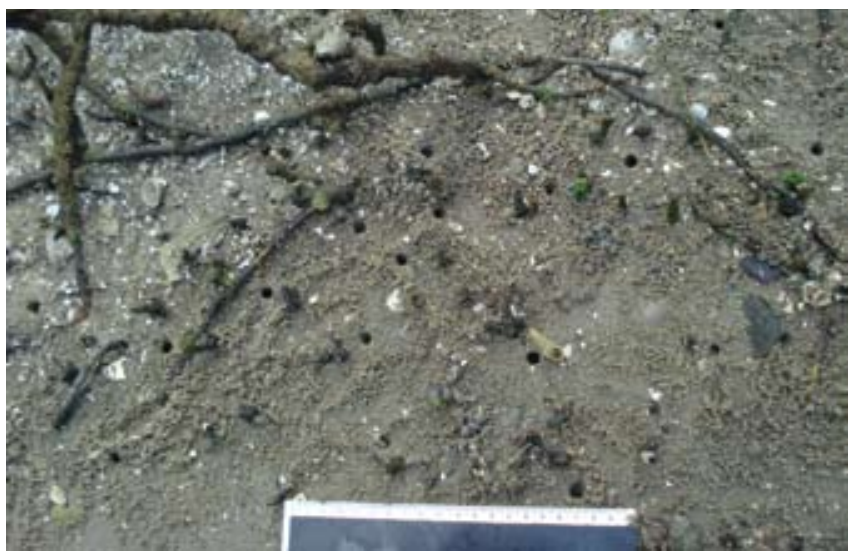

Figura 15. Baía do Araçá: aberturas de galerias do crustáceo Uca, região entremarés, núcleo de manguezal localizado próximo à Ilha Pernambuco. Foto: A.C.Z. Amaral (07/2009).

Figure 15. Araçá Bay: burrow opening of the crab Uca, intertidal zone, mangrove vegetation near Pernambuco Island. Photo: A.C.Z. Amaral (07/2009).

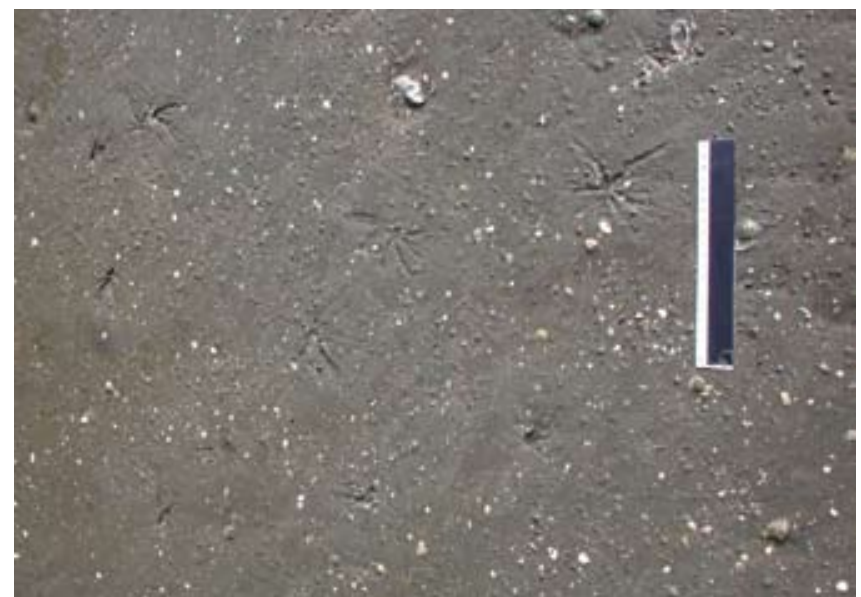

Figura 16. Baía do Araçá: marcas do bivalve Macoma em sedimento arenolamoso na região entremarés. Foto: S.A. Nallin (05/2009).

Figure 16. Araçá Bay: signs of the clam Macoma in muddy sand flat, intertidal zone. Photo: S.A. Nallin (05/2009).

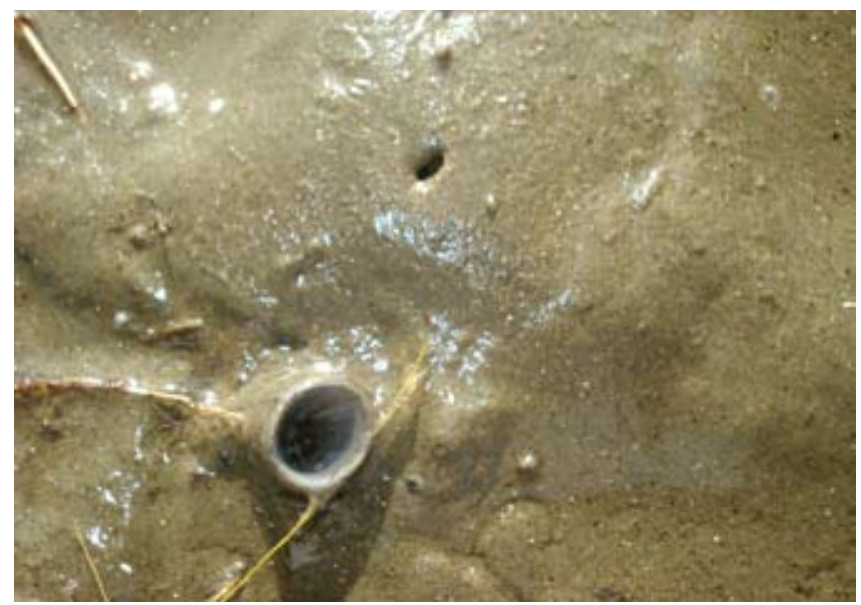

Figura 17. Baía do Araçá: tubo do poliqueta Diopatra aciculata em sedimento areno-lamoso na região entremarés. Foto: A.C.Z. Amaral (07/2009).

Figure 17. Araçá Bay: tube of the polychaete Diopatra aciculata in muddy sand flat, intertidal zone. Photo: A.C.Z. Amaral (07/2009).

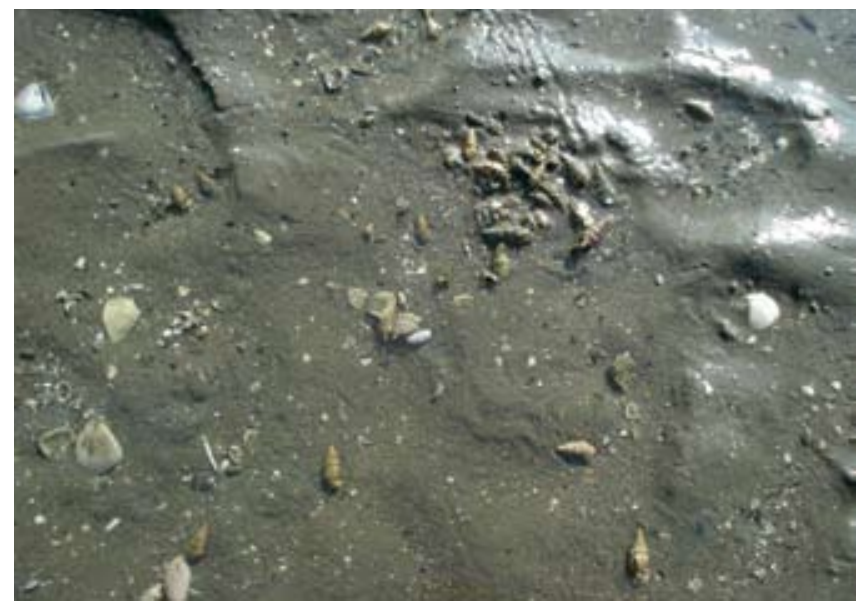

Figura 18. Baía do Araçá: gastrópode Cerithium atratum na região entremarés. Foto: A.C.Z. Amaral (07/2009).

Figure 18. Araçá Bay: the gastropod Cerithium atratum, intertidal zone. Photo: A.C.Z. Amaral (07/2009). 
área de baixíssimo grau de compactação, difícil drenagem e forte odor, características de ambiente pobre em oxigênio (Belúcio 1995).

Esse soterramento ocorreu em vários locais da baía, alterando a topografia, a circulação hídrica e, consequentemente, as características sedimentológicas como um todo, conforme observado por Lopes (1993) e Belúcio (1995), ao estudarem a macrofauna dessa área logo após a instalação do emissário. Com a entrada em operação do emissário, em 1990, constatou-se graves contaminações oriundas de esgoto doméstico, como concentrações elevadas de carbono orgânico e de matéria orgânica em decomposição, com a formação de zonas anóxicas (Gubitoso et al. 2008).

A esses problemas soma-se o crescimento populacional provocado pela ocupação desordenada da cidade decorrente do intenso processo de crescimento populacional vivido pela região entre 1985 (24.534) e 2008 (69.772), conforme SEADE (2009). O entorno da Baía do Araçá seguiu essa tendência, a qual, associada à falta de serviços públicos de saneamento, levou a uma aceleração da descaracterização desse ambiente, com lixo e entulho sendo depositado em suas margens, associado ao aporte de esgoto doméstico.

Embora desgastado e empobrecido, o Araçá continua vivo à espera de medidas que tornem possível a recuperação sócio ambiental da baía e do seu entorno. Vale destacar que manguezais são considerados produtores de bens e serviços extremamente frágeis. Como produtor primário constitui recurso rentável finito principalmente quando se leva em conta a produção natural de mariscos, ostras, camarões, caranguejos, siris, peixes, entre outros (Schaeffer-Novelli 2000). O Araçá, apesar das alterações impostas, permanece como um verdadeiro "laboratório natural"; conforme as pesquisas prosseguem, sua biodiversidade e alta produtividade continuam sendo reveladas. Atualmente, por exemplo, um catador de mariscos coleta $20 \mathrm{~L}$ do molusco bivalve Anomalocardia brasiliana (berbigão), em um período de 2 horas (Figura 19a-b).

\section{A importância da Baía do Araçá}

\section{Biodiversidade}

No sudeste do Brasil há conhecimentos consistentes sobre a distribuição dos bosques de mangue, produção de serrapilheira e decomposição do material vegetal em toda região, mas quase nada se sabe sobre a fauna desses mesmos manguezais, salvo algumas exceções.
No entanto, os organismos da Baía do Araçá, principalmente a fauna, são relativamente bem conhecidos. Os primeiros artigos publicados em revistas científicas são de Sawaya $(1950,1951)$. Um deles, intitulado "Balanoglossus gigas Fr. Müller rediscovered on the Brazil coast", foi publicado no mais conceituado periódico estrangeiro, a revista "Nature" (Apêndice 2). Inúmeros trabalhos científicos foram realizados desde então, enfocando os mais variados aspectos dos organismos lá presentes. Entretanto, alguns dos estudos realizados revelam uma diminuição em termos de riqueza e densidade faunística após as supracitadas intervenções humanas (Belúcio 1995, Lopes 1993, Arruda 2000). A despeito dessas alterações, relictos da área originalmente ocupada por manguezais se mantiveram até o presente permitindo que a vitalidade e a importância social e ecológica dessa baía sejam ainda atestadas.

Nesse ambiente tão especial, onde a diversidade de habitats é surpreendente, a biodiversidade conhecida, isto é formalmente registrada por pesquisadores, alcança 733 espécies, das quais 34 foram descritas como novas para a ciência. O descobrimento de novas espécies nessa região - como a descrição recente de mais uma nova espécie de Polychaeta para o Araçá, Arabella aracaensis por Steiner \& Amaral (2009) - continua acontecendo, o que reafirma a sua peculiaridade ambiental e excepcional importância científica em nível mundial.

A seguir é apresentada uma síntese sobre a flora e a fauna da Baía do Araçá, cuja lista por táxon encontra-se no Apêndice 1 .

\section{Flora}

Nos manguezais das Américas e do oeste da África, os bosques de mangue são caracterizados por reduzido número de espécies arbóreas típicas. Entretanto, essa baixa diversidade específica é plenamente compensada por elevada diversidade funcional. Esses vegetais superiores são os poucos capazes de ocupar sedimentos areno-lamosos, com baixos teores de oxigênio e áreas sujeitas a amplas variações de salinidade. No Brasil, são comuns as angiospermas dos gêneros Rhizophora, Avicennia e Laguncularia. No Araçá as espécies dominantes são Avicennia schaueriana (mangue-preto, siriúba) e Laguncularia racemosa (mangue-branco), inclusive com a presença de indivíduos jovens, evidenciando a vitalidade dos bosques com potencial para expansão. Além dessas duas espécies mais comuns,
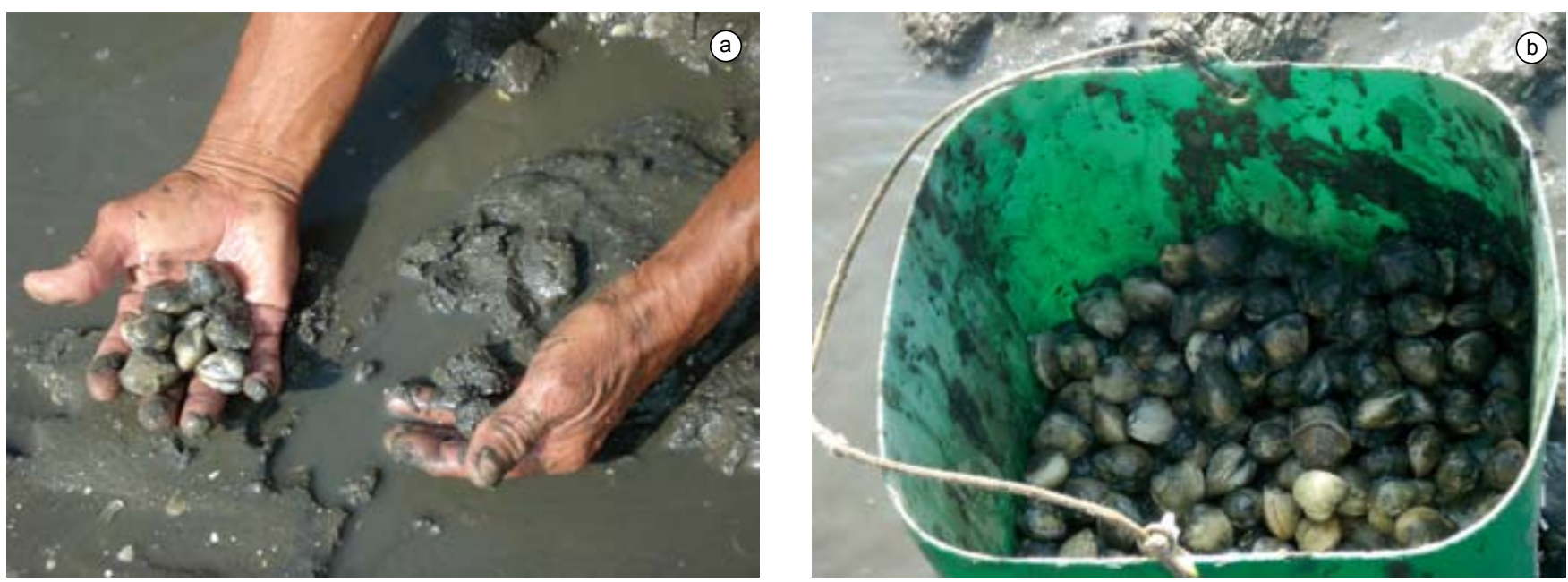

Figura 19. Baía do Araçá: a) caiçara coletando o berbigão Anomalocardia brasiliana em sedimento areno-lamoso na região entremarés; b) resultado da coleta de A. brasiliana em poucas horas de trabalho. Foto: A.C.Z. Amaral (07/2009).

Figure 19. Araçá Bay: a) a Caiçara (native inhabitants in the Southeastern coast of Brazil) hand digging the bivalve Anomalocardia brasiliana out of the muddy sand flat, intertidal zone; b) clams (A. brasiliana) hand caught in a few hours of work. Photo: A.C.Z. Amaral (07/2009). 
nota-se, ainda, a presença de alguns exemplares de Rhizophora mangle (mangue-vermelho). Os três principais núcleos demonstram estar em expansão, dois deles situados na parte superior da praia, um com cerca de $320 \mathrm{~m}^{2}$ e o outro com $2.000 \mathrm{~m}^{2}$, e um terceiro, com $1.000 \mathrm{~m}^{2}$, localizado próximo à parte inferior da zona de marés e da Ilha Pernambuco (Figuras 3-8).

Na Baía do Araçá, assim como ocorre na região sudeste, várias áreas de manguezais são marginais à Mata Atlântica, sendo comum a presença de macrófitas superiores aquáticas. Nos ambientes marinhos, as pradarias de Halodule e Ruppia são pouco comuns ao longo da costa sudeste. No entanto, no Araçá ocorre uma pradaria de Halodule emarginata, espécie descrita originalmente a partir de exemplares provenientes desse local.

Entre as macroalgas que geralmente ocorrem sobre os troncos, pneumatóforos e rizóforos (raízes aéreas) das árvores de mangue, como também em afloramentos rochosos, os grupos das Bostrichietum (Bostrichia) e Enteromorpha são os mais frequentes. No sedimento podem ocorrer clorófitas, como Rhizoclonium, Enteromorpha, Ulva e Cladophora (Apêndice 1). A microflora é também, em geral, muito abundante, porém pouco estudada no Brasil.

\section{Fauna}

As peculiaridades da Baía do Araçá agregam características ambientais diversas, representadas pela presença de vegetação de manguezal e de uma planície de maré areno-lamosa relativamente extensa que propicia condições para a ocorrência de organismos raramente representados em outros ambientes costeiros da região. Devido a essas condições específicas, muitas espécies desenvolvem populações numerosas e de elevada biomassa, assumindo, portanto, grande importância ecológica. A seguir, a fauna do Araçá será apresentada e comentada segundo seus hábitos de vida:

\subsection{Espécies que ocupam as áreas de manguezais}

Como parte desse grupo, são bem representados o caracol-da-folha, Littorina angulifera, e os caranguejos, como Uca spp. (Figura 15).

\subsection{Espécies visitantes terrestres - aquelas que habitam o} ambiente terrestre, mas que visitam periodicamente a baía à procura de alimento

Nesse grupo, os principais representantes são as aves, como as garças, biguás, quero-queros, maçaricos, gaivotas, martinspescadores, bem-te-vis e gaviões que são frequentes na região. Aves migratórias passam por essa região durante algumas semanas por ano, como os talha-mares, colhereiros e os trinta-réis, estes últimos nidificando em vários pontos do Canal de São Sebastião. Pequenos mamíferos são frequentemente avistados se alimentando nos núcleos de manguezal e áreas adjacentes, dentre os quais se destacam os morcegos pescadores.

\subsection{Espécies marinhas que passam parte de seu ciclo de vida nos manguezais}

Bons exemplos desse grupo, dada a importância econômica, são o camarão-branco Penaeus schimitti e o sete-barbas Xiphopenaeus kroyeri, e várias espécies de peixes, como Cynoscion jamaicensis (pescada), Micropogonias furnieri (corvina), Eucinostomus argenteus (carapicú), Epinephelus spp. (garoupa), Paralichthys spp. (linguado), Haemulon steindachneri (corcoroca), Diapterus rhombeus (carapeva) e Diplectrum radiale (mixole), comuns na região, conforme Pires-Vanin et al. (1997) e Rossi-Wongtschowski et al. (1997).
3.4. Espécies próprias dos substratos rochosos e, principalmente, do sedimento - macrofauna bentônica

Esse grupo inclui uma grande diversidade de organismos (Figuras 9-19a), alguns dos quais em grande abundância, e representados por várias espécies de Protozoa, Porifera, Cnidaria, Bryozoa, Mollusca, Nemertea, Polychaeta, Sipuncula, Echiura, Entoprocta, Pycnogonida, Crustacea, Echinodermata, Ascidiacea e Enteropneusta (Tabela 1 e Apêndice 1). Com certeza, essa fauna bentônica, considerando a parte interna da baía e a mais externa, até cerca de $10 \mathrm{~m}$ de profundidade, está entre as mais estudadas no Estado e, provavelmente, no País.

Entre as 641 espécies da macrofauna registradas para o Araçá (Tabela 1, Apêndice 1), tem-se: $32 \%$ de poliquetas, $28 \%$ de moluscos e $17 \%$ de crustáceos. Esses animais são importantes como fonte de alimento, diretamente para o homem ou para outros organismos carnívoros e onívoros que deles se alimentam. Cabe destacar ainda, o importante papel dos herbívoros, como alguns crustáceos e moluscos, na fragmentação do material vegetal, disponibilizando-o para outros níveis da cadeia trófica. Outra relevante função desempenhada por esses organismos diz respeito à bioturbação, favorecendo a reoxigenação do sedimento (geralmente pobre em oxigênio), auxiliando na decomposição da matéria orgânica e na disponibilização de nutrientes para todo o sistema.

\subsubsection{Os poliquetas}

Os poliquetas estão entre os mais abundantes e diversificados organismos bentônicos de fundos moles e, no Araçá, onde foram registradas 207 espécies, constituem o grupo melhor representado tanto em composição específica quanto em densidade.

Tabela 1. Número de espécies (plantas e invertebrados) por táxons superiores, registradas para a Baía do Araçá, incluindo as novas e ameaçadas (1950-2009).

Table 1. Number of species (plants and invertebrates) in superior ranks, recorded from the Araçá Bay, including new and endangered ones (1950-2009).

\begin{tabular}{lrcc}
\hline \multirow{2}{*}{ Táxons } & \multicolumn{3}{c}{$\mathbf{N}^{\mathbf{0}}$ de Espécies } \\
\cline { 2 - 4 } Chlorophyta & Total & Novas & Ameaçadas \\
Rodophyta & 23 & - & - \\
Phaeophyta & 46 & - & - \\
Magnoliophyta & 13 & - & - \\
Ciliophora & 4 & 1 & - \\
Porifera & 6 & - & - \\
Cnidaria & 12 & 1 & - \\
Nemertea & 32 & - & - \\
Mollusca & 1 & - & - \\
Polychaeta & 179 & 1 & - \\
Echiura & 207 & 17 & 2 \\
Sipuncula & 3 & 1 & - \\
Pycnogonida & 10 & - & - \\
Crustacea & 1 & - & - \\
Kamptozoa (Entoprocta) & 4 & 2 & - \\
Bryozoa (Ectoprocta) & 40 & - & - \\
Echinodermata & 18 & - & - \\
Enteropneusta & 4 & 1 & 2 \\
Ascidiacea & 18 & 1 & 5 \\
Total Geral & 733 & 34 & 9 \\
\hline & & &
\end{tabular}


Em virtude do papel ecológico que desempenham, os poliquetas têm uma importância econômica indireta, por participarem da cadeia trófica que envolve animais de valor comercial, promovendo a produção de recursos exploráveis, tais como peixes, camarões, siris e caranguejos, além de várias espécies de aves. Conforme Amaral et al. (1994), peixes como linguado, corvina, maria-luiza e pernade-moça se alimentam quase que exclusivamente de poliquetas, os quais chegam a constituir de 70 a $80 \%$ do total de itens do conteúdo estomacal desses peixes. Alguns poliquetas são também considerados indicadores, sendo vários deles potenciais bioindicadores de áreas poluídas (Amaral \& Morgado 1994).

Os poliquetas abundantes e frequentes no Araçá são representados pelas espécies Heteromastus filiformis, Laeonereis culveri e o Complexo Capitella capitata. Algumas espécies (Isolda pulchella, Armandia agilis, Glycinde multidens e Parandalia americana) destacam-se principalmente pela frequência com que ocorrem. Outras menos abundantes, como Diopatra cuprea e Eunice sebastiani, são comumente utilizadas como isca para pesca esportiva.

\subsubsection{Os moluscos}

Entre as 179 espécies com registro para o Araçá, têm-se 78 Gastropoda, 96 Bivalvia e 5 Polyplacophora. De um modo geral, os gastrópodes são pouco comuns em substratos moles na região entremarés. Além de Olivella minuta, são também frequentes e abundantes Cerithium atratum e Nassarius vibex. Entre os bivalves, espécies mais resistentes, como Iphigenia brasiliana (taioba), Anomalocardia brasiliana (berbigão), Corbula caribaea e Lucina pectinata (lambreta), mantiveram o padrão de dominância, mesmo após as obras do emissário, mas com menores densidades. Essas espécies são bioindicadoras de poluição orgânica, embora ocorram apenas em locais com sinais de enriquecimento orgânico. Nota-se também a presença de outros mariscos comestíveis, como Tagelus plebeius (unha-de-velho) e Tivela mactroides (berbigão). No momento, o gênero Corbula vem sendo estudado, utilizando-se técnicas moleculares, a partir de exemplares procedentes do Araçá (Quast et al. 2009).

O bivalve Anomalocardia brasiliana, uma das espécies dominantes na região é conhecido popularmente como berbigão, sarro-de-pito, marisco-pedra, mija-mija ou vôngole. Vive enterrado a aproximadamente $5 \mathrm{~cm}$ da superfície em fundos areno-lamosos, de águas rasas e calmas (Schaeffer-Novelli 1976), sendo encontrado em abundância na região entremarés de praias e estuários, onde é facilmente capturado manualmente (Figura 19) ou com auxílio de rastelo. Devido ao valor nutritivo é uma espécie bastante explorada, tanto comercialmente por caiçaras e pela população local, quanto por turistas, que a utilizam para consumo próprio (Amaral et al. 1990). Tobias \& Silva (1957) fazem referência aos berbigões como boa fonte de proteína, embora o teor de lipídeos seja baixo, enquanto Maltz \& Faerman (1958) relatam a presença de ferro no produto fresco. Maciel (1986) também comenta sobre o alto valor proteico dessa espécie, sendo que em cada $100 \mathrm{~g}$ de carne tem-se 13,81 g de proteína e 21,6 mg de ferro. Em pesquisa que está sendo desenvolvida desde 2008 no Araçá, sobre o crescimento e reprodução de $A$. brasiliana, foi registrada uma densidade média de 51,07 indivíduos $/ \mathrm{m}^{2}$ (Corte et al. 2009).

Entre os bivalves de substrato duro é marcante a presença da ostra-do-mangue Crassostrea rhizophorae e dos mexilhões Brachidontes spp. e Perna perna.

\subsubsection{Os crustáceos}

Do total de espécies registradas para o Araçá, 112 são crustáceos. O caranguejo chama-maré ( $U c a$ spp.) é abundante na parte superior da região entremarés e, principalmente, nos núcleos de mangue, onde o número de tocas é de cerca de $80 / \mathrm{m}^{2}$ (Figura 15). As espécies de Uca são consideradas comedoras de depósitos, consumindo uma mistura de microalgas bentônicas e detritos. São, por isso, muito importantes como disponibilizadoras de nutrientes para a superfície do sedimento.

A região do Araçá favorece o estabelecimento de espécies de crustáceos, assim como de outros grupos zoológicos, com o hábito de construir galerias e que se alimentam do material trazido pelo fluxo de água, como é o caso de Upogebia e Kalliapseudes, organismos comuns nessa região. Kalliapseudes é um tanaidáceo abundante no Araçá, que comumente ocorre em estuários e planícies de marés areno-lamosas da região sudeste-sul. Outros vivem sobre o sedimento (epifauna), alimentando-se diretamente dos detritos orgânicos depositados, como os siris do gênero Callinectes, os caranguejos Menippe, Eriphia, Panopeus, Pachygrapsus e Petrolistes, e os ermitões Pagurus criniticornis, Clibanarius vittatus, C. sclopetarius e C. antillensis, todos abundantes e frequentes no Araçá.

\section{Destaques da Fauna}

\section{Espécies novas}

Entre o total de táxons registrados para o Araçá, 2 gêneros e 34 espécies foram descritos como novos para a ciência com base em material proveniente dessa baía, muitas das quais não foram ainda encontradas em outras localidades. Os exemplares tipos dessas espécies de Magnoliophyta (1), Porifera (1), Gastropoda (1), Polychaeta (17), Echiura (1), Entoprocta (2), Crustacea (9), Ascidiacea (1) e Enteropneusta (1) encontram-se depositados em diferentes museus nacionais e estrangeiros, como Museu de Zoologia da Universidade de São Paulo, Museu Nacional do Rio de Janeiro, Museu de Zoologia da Universidade Estadual de Campinas e Smithsonian National Museum of Natural History (Washington, DC, USA).

\section{Espécies ameaçadas de extinção}

Duas espécies de poliquetas com registro no Araçá, Eunice sebastiani e Diopatra cuprea (Figura 17), constam da "Fauna Brasileira Ameaçada de Extinção" (Amaral et al. 2008). Essas espécies, que ocorrem na parte inferior da região entremarés em sedimentos arenolamosos, são utilizadas ou exploradas com objetivos comerciais, sobretudo na crescente indústria de isca para pesca, enquanto outras também são usadas para atividades de aquariofilia. Eunice sebastiani alcança cerca de $2 \mathrm{~m}$ de comprimento e tem registro confirmado, até o momento, apenas para o Estado de São Paulo. Diopatra cuprea vem sendo caracterizada como um complexo de espécies, representada no Brasil por cinco diferentes morfotipos (Steiner 2005).

Dentre os equinodermos, cinco espécies de estrela-do-mar que ocorrem no Araçá estão incluídas nesta lista de espécies ameaçadas: Asterina stellifera, Astropecten brasiliensis, A. marginatus, Luidia clathrata e L. senegalensis. Essas são apanhadas por turistas e aquariofilistas, que as comercializam ou utilizam como artefatos decorativos ou religiosos. Uma vez que algumas dessas estrelas-do-mar vivem em fundos arenosos e/ou lamosos, uma forte ameaça é a captura acidental por redes de arrasto de pesca.

O enteropneusta gigante Willeya loya, descrito para o Araçá e Siriúba (Ilhabela) e ainda não encontrado em nenhum outro local, também está incluído entre as espécies ameaçadas de extinção na Lista Nacional. Balanoglossus gigas, outro enteropneusta registrado para o Araçá, está na Lista das Espécies Ameaçadas de Extinção do Estado de São Paulo. Descrita por Spengel (1893), baseado em material coletado por Fritz Müller, no litoral de Santa Catarina (1884-1885), trata-se de uma espécie vulnerável aos impactos antrópicos. Embora B. gigas tenha se tornado rara na Baía do Araçá após a construção do emissário de esgotos (Rodrigues 1999), nos últimos anos sua população vem crescendo gradativamente. 
Além da constatação da diminuição das populações por excesso de captura, as espécies ameaçadas sofrem também o impacto de outros fatores de origem antrópica, sobretudo a descaracterização ambiental em conseqüência das constantes alterações físicas pelas quais o Araçá e o seu entorno têm sido submetidos.

\section{Espécies bioindicadoras}

A composição da fauna e flora diz muito a respeito do grau de estabilidade ou perturbação de um ambiente. Muitas espécies só ocorrem em locais mais estáveis, enquanto outras, oportunistas, aproveitam espaços vazios disponibilizados na comunidade, após perturbações de diversas origens. As espécies oportunistas frequentemente indicam o estado de perturbação dos ambientes. Comunidades bentônicas, organismos que vivem sobre o substrato ou enterrados, têm sido utilizadas como uma das principais ferramentas para avaliação da qualidade ambiental.

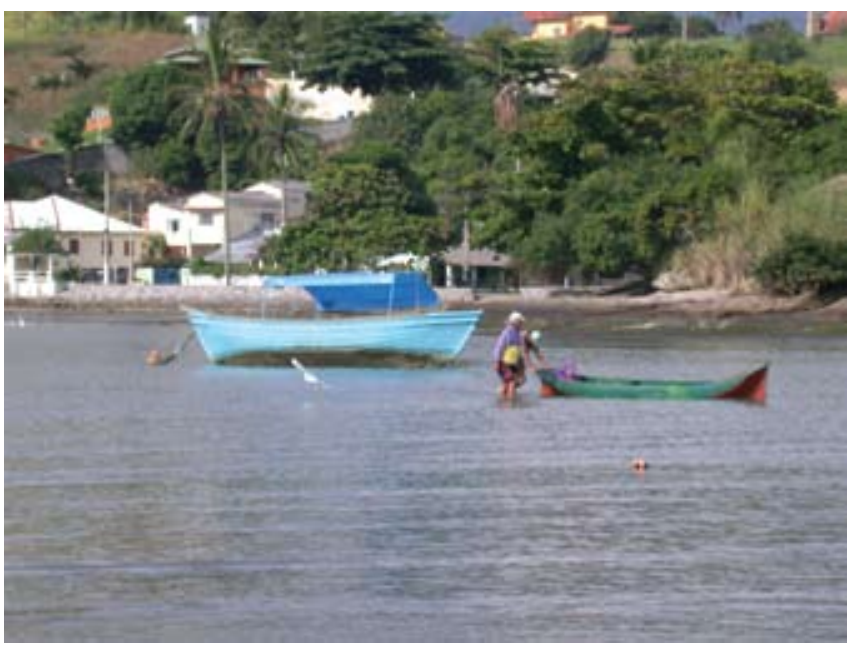

Figura 20. Baía do Araçá: pescadores locais retornando de uma pescaria na baía. Foto: S.A. Nallin (05/2009).

Figure 20. Araçá Bay: local fishermen returning from a fishing trip in the bay. Photo: S.A. Nallin (05/2009).
O conhecimento da diversidade e dominância desses organismos mostra como são explorados recursos como espaço e alimento. A descrição do comportamento temporal desses padrões permite, ainda, identificar a ocupação diferencial dos recursos, ao longo do tempo, ou distinguir os mecanismos pelos quais a comunidade desenvolveu os padrões atuais. Quando ocorre uma perturbação de qualquer origem, uma comunidade tende a ter sua estrutura modificada em diferentes níveis. A evolução da modificação temporal dos padrões de dominância, comparados aos registrados em ambientes semelhantes, pode fornecer indícios de uma recuperação ou indicar os mecanismos por meio dos quais a comunidade está se adaptando às novas condições impostas ao ambiente.

Nesse contexto, alguns crustáceos, moluscos e, sobretudo, poliquetas constituem excelentes bioindicadores. Espécies oportunistas, associadas ao enriquecimento orgânico, abundantes no Araçá, como os poliquetas Heteromastus filiformis e complexo Capitella capitata e o crustáceo Kalliapseudes schubarti, estão incluídas entre as mais utilizadas para esse tipo de avaliação das condições ambientais (Amaral et al. 2003).

\section{Espécies como recursos naturais}

Na Baía do Araçá são exploradas várias espécies de moluscos, como Anomalocardia brasiliana, Crassostrea rhizophorae, Iphigenia brasiliana, Lucina pectinata, Macoma constricta, Neoteredo reynei, Perna perna, Tagelus plebeius e Tivela mactroides, e de crustáceos, como Xiphopenaeus kroyeri, Callinectes danae, Penaeus spp., sendo as mais comuns A. brasiliana e C. rhizophorae. Nos dias de marés suficientemente baixas, são frequentes os catadores de mariscos e siris cujo produto é comercializado em feiras livres, além de servirem para consumo próprio. É comum, também, a presença de pescadores artesanais, que utilizam picarés e tarrafas durante as marés baixas, ou pescam embarcados em pequenas canoas (Figura 20), capturando camarões e peixes, como garoupa, corvina, corcoroca, mixole, carapicú, carapeva, linguado.

\section{Conservando esse ambiente único - O Araçá}

Há décadas a ciência vem desvendando a diversidade específica e funcional da Baía do Araçá, berço de espécies novas para a ciência e de outras ameaçadas de extinção, além de fonte de recursos alimentares para a população local. Além de todos os importantes registros de

Tabela 2. Trabalhos publicados sobre organismos procedentes da Baía do Araçá (1950-2009).

Table 2. Published works about organisms from Araçá Bay (1950-2009).

\begin{tabular}{|c|c|c|c|}
\hline \multirow[t]{2}{*}{ Tipo } & \multicolumn{3}{|c|}{ Publicações } \\
\hline & Nacional & Estrangeira & Total \\
\hline - Artigos em Revistas Indexadas & 47 & 80 (2 Nature) & 127 \\
\hline - Livros e Capítulos de Livros & 5 & & 5 \\
\hline - Trabalhos Completos e Resumos Expandidos Publicados em Anais de Congressos & 3 & 17 & 20 \\
\hline \multirow[t]{2}{*}{ - Comunicações Publicadas em Resumos de Congressos } & 108 & 22 & 130 \\
\hline & Concluídas & Em Andamento & \\
\hline - Iniciação Científica & 2 & - & 2 \\
\hline - Conclusão de Curso / Monografia & 11 & - & 11 \\
\hline - Dissertação de Mestrado & 33 & 1 & 34 \\
\hline - Tese de Doutorado & 22 & 2 & 24 \\
\hline - Pós-Doutorado & 1 & 2 & 3 \\
\hline - Livre-Docência & 1 & - & 1 \\
\hline - Relatórios Técnicos & 2 & - & 2 \\
\hline - Textos Web & 1 & - & 1 \\
\hline - Jornais e Revistas & 47 & - & 47 \\
\hline
\end{tabular}


estudos realizados desde 1950, que somam 127 artigos publicados em revistas nacionais e estrangeiras, 150 trabalhos em eventos (incluindo artigos completos publicados em anais e resumos expandidos), 1 livro, 4 capítulos de livros e 77 títulos de teses, dissertações, monografias, entre outros, além de vários textos de revistas e jornais (Tabela 2, Apêndice 2), conta-se também com o depoimento de caiçaras que vivem no Araçá ou que dele dependem. Esses estudos incluem temas voltados principalmente para a diversidade da macrofauna, alterações provocadas por ação antrópica, genética evolutiva, desenvolvimento, fisiologia, parasitologia e dinâmica populacional.

As características excepcionais da Baía do Araçá, que atraem a atenção de cientistas de todo mundo, a transformaram também num verdadeiro laboratório a céu aberto para atividades de Educação Ambiental e para cursos do ensino superior, como oceanografia, ciências biológicas, gestão ambiental, dentre outros.

Os dados científicos acumulados em mais de 50 anos de pesquisa e os depoimentos da comunidade revelam o quanto a conservação dessa região representa para a Ciência e para a vida de quem dela depende. Nesse sentido, é inquestionável a necessidade premente de se proteger as espécies que teimam em manter a preciosa riqueza da Baía do Araçá. Atitudes, como a prática da educação ambiental, por exemplo, que considerem o Araçá como patrimônio científico, podem sinalizar para a sociedade que de fato as decisões governamentais estão alinhadas na direção da sustentabilidade.

As constantes e variadas ameaças à existência da baía, por meio de projetos que pretendem sua eliminação parcial ou total, e o crescente estado de degradação sofrido pela região, em particular, e pelo Canal de São Sebastião, em geral, comprovam a necessidade de se estabelecer uma política de uso racional dessa área.

Assim, é necessário que o estado implemente uma Unidade de Conservação nessa área e que estabeleça seu plano de manejo: 1) ouvindo cientistas e ambientalistas para conservação e recuperação da Baía do Araçá e de seu manguezal, bem como do seu entorno; 2) incluindo educação ambiental e capacitação técnica apropriada, com estabelecimento de um Centro de Visitação e de Educação Ambiental que receba e oriente estudantes e turistas; 3) investindo em pesquisas sobre a biodiversidade local e em educação, fomentando a publicação de inventários, de guias e manuais sobre a biota da região; e 4) promovendo estudos da hidrodinâmica da baía e adjacências, como também do solo, para alimentar de forma correta um plano de recuperação da área e de ocupação de seu entorno.

Segundo a legislação vigente, por abrigar ambientes característicos de manguezal a Baía do Araçá é Área de Preservação Permanente, sendo considerada Patrimônio Nacional, nos termos do artigo 225, parágrafo $4^{\circ}$, da Constituição do Brasil (05/10/1988). Ademais, o ecossistema manguezal, em toda a sua extensão, encontra-se incluído em diversos diplomas legais, como leis, decretos e resoluções que impõem ordenação de uso e ações a essas áreas. A Lei Federal $\mathrm{N}^{\circ}$ 4.771/65, mais conhecida como Código Florestal, com algumas alterações que foram efetuadas no decorrer nos últimos anos, busca proteger o mangue contra qualquer tipo de uso que remova a vegetação, os quais são considerados Áreas de Preservação Permanente (APP). Assim, o poder público deve adotar medidas que visem a sua proteção, seguindo também a recomendação dos especialistas que "onde quer que existam representantes desse ecossistema sua conservação deve ser prioritária" (Dinerstein et al. 1995, Olson et al. 1996). Ademais, as praias localizadas dentro da baía também são protegidas por lei, dado seu caráter de bem da união (Constituição Federal; Artigo 20), de uso comum do povo (Lei 7.661/88; Artigo 10), proteção esta que também é assegurada pela Lei Orgânica do Município de São Sebastião, que, em seu Artigo 155, inclui entre as áreas de proteção permanente "os manguezais; aquelas que abrigam exemplares raros de fauna e flora e as que servem de local de pouso ou reprodução de migratórios; e as paisagens notáveis".

A possível e desejada relação harmônica entre turismo sustentado e ambiente dificilmente floresce em zonas portuárias e industriais. Inserida em uma das mais belas e conservadas regiões litorâneas do país, de inegável vocação turística, o Poder Público tem a responsabilidade de manter íntegro o frágil patrimônio ambiental sob sua tutela, para usufruto da presente e das futuras gerações, dando o exemplo de como uma área pode ser revitalizada e integrada ao ambiente urbanizado.

\section{Agradecimentos}

Aos pesquisadores Bruno C. Vellutini, Carl Thurman, Carlo Magenta Cunha, Cláudio G. Tiago, Diclá P. Santos, Eduardo Hajdu, Emerson C. Mossolin, Estela M. Plastino, Eurico C. de Oliveira Filho, Fernando L. Mantelatto, Fábio Lang da Silveira, Flávio D. Passos, Inácio D. da Silva Neto, João M.M. Nogueira, John C. McNamara, José Eduardo .A.R. Marian, Leandro M. Vieira, Luiz R.L. Simone, Marcelo V. Fukuda, Márcio R. Custódio, Marcos D.S. Tavares, Mariana Capparelli, Michela Borges, Roberto M. Shimizu, Rosana M. Rocha, Sérgio N. Stampar, Tatiana M. Steiner e Terue C. Kihara pelo envio de informações e auxílio na revisão das listas taxonômicas. À bióloga Silvana A.H. Nallin pelo auxílio na digitação e revisão dos dados.

\section{Referências}

AMARAL, A.C.Z. \& MORGADO, E.H. 1994. Alteraciones en la fauna de anélidos poliquetos de Araçá, São Sebastião (SP - Brasil). Rev. Acad. Colomb. Cienc. 19(72):147-152.

AMARAL, A.C.Z., DENADAI, M.R., TURRA, A. \& RIZZO, A.E. 2003. Intertidal macrofauna in Brazilian subtropical sandy beaches landscape. J. Coast. Res. 35:446-455.

AMARAL, A.C.Z., MORGADO, E.H., LOPES, P.P., BELÚCIO, L.F., LEITE, F.P.P. \& FERREIRA, C.P. 1990. Composition and distribution of the intertidal macrofauna of sandy beaches on São Paulo coast. In Anais II Simpósio de Ecossistemas da Costa Sul e Sudeste Brasileira - Estrutura, Função e Manejo, Publ. ACIESP, São Paulo, p. 258-279.

AMARAL, A.C.Z., NONATO, E.F. \& PETTI, M.A.V. 1994. Contribution of the polychaetous annelids to the diet of some Brazilian fishes. Memoir. Mus. Natl. Hist. 162:331-337.

AMARAL, A.C.Z., VOLKMER-RIBEIRO, C., MANSUR, M.C.D., SANTOS, S.B., AVELAR, W.E.P., MATTEWS-CASCON, H., LEITE, F.P.P., MELO, G.A.S., COELHO, P.A., BUCKUP, G.B., BUCKUP, L., VENTURA, C.R.R. \& TIAGO, C.G. 2008. A Situação de Ameaça dos Invertebrados Aquáticos no Brasil. In Livro Vermelho da Fauna Brasileira Ameaçada de Extinção. (A.B.M. Machado, G.M. Drummond \& A.P. Paglia, orgs). Ministério do Meio Ambiente - MMA, Brasilia (DF). v. 1, p. $156-351$.

ARRUDA, E.P. 2000. Moluscos da região entremarés de praias arenosas do litoral norte do Estado de São Paulo (SP). Fatores afetando a composição e distribuição específica; Grupos funcionais de alimentação. Dissertação de Mestrado, Universidade de São Paulo, São Paulo.

BELÚCIO, L.F. 1995. Comunidades bênticas de moluscos da zona entremarés da região do Araçá, São Sebastião, SP. Dissertação de Mestrado, Universidade Estadual de Campinas, Campinas.

CGG. 1919. Exploração do Rio Juqueryquerê. 2 ed. Comissão Geographica e Geologica do Estado de São Paulo, São Paulo.

CIMA. 1991. Subsídios técnicos para elaboração do relatório nacional do Brasil para a Conferência das Nações Unidas sobre o Meio Ambiente e Desenvolvimento. Comissão Interministerial para a preparação da preparação a Conferência das Nações Unidas sobre o Meio Ambiente e Desenvolvimento, Brasília (DF).

CORTE, G.N., YOKOYAMA, L.Q. \& AMARAL, A.C.Z. 2009. Distribuição e abundância de Anomalocardia brasiliana em praias do Litoral Norte 
do Estado de São Paulo. In II Congresso Brasileiro de Biologia Marinha. Associação Brasileira de Biologia Marinha, Rio de Janeiro, p. 578-583. (CDRom)

DIEGUES, A.C.S. 1987. Conservação e desenvolvimento sustentado dos ecossistemas litorâneos no Brasil. In Simpósio sobre Ecossistemas da Costa Sul e Sudeste Brasileira. ACIESP, São Paulo, p. 196-243.

DINERSTEIN, E., OLSON, D.M., GRAHAM, D., WEBSTER, A., PRIMM, S., BOOKBINDER, M. \& LEDEC, G. 1995. A conservation assessment of the terrestrial ecoregions of Latin America and the Caribbean. The Word Bank in association with The World Wildlife Fund, Washington, D.C., USA.

FRANCISCO, J. \& CARVALHO, P.F. 2003. Desconstrução do lugar - o aterro da praia da frente do centro histórico de São Sebastião - SP. In Ambientes - estudos de Geografia (L.H. Oliveira, org.). AGETEO, Rio Claro. p. 105-119. http://www.rc.unesp.br/igce/planejamento/gpapt/ artigos.htm (último acesso em 09/06/2009).

GUBitoso, S., DULEBA, W., TEODORO, A.C., PRADA, S.M., ROCHA, M.M., LAMPARELLI, C.C., BEVILACQUA, J.E. \& MOURA, D.O. 2008. Estudo geoambiental da região circunjacente ao emissário submarino de esgoto do Araçá, São Sebastião, SP. Rev. Bras. Geocienc. 38(3):467-475.

LAMPARELLI, C.C., MOURA, D.O., VINCENT, R.C., RODRIGUES, F.O., LOPES, C.F. \& MILANELLI, J.C.C. 1998. Mapeamento dos ecossistemas costeiros do Estado de São Paulo. Secretaria do Meio Ambiente, CETESB, São Paulo.

LOPES, P.P. 1993. Estrutura da comunidade de poliquetos da zona entremarés da região do Araçá, São Sebastião, SP. Dissertação de Mestrado, Universidade Estadual de Campinas, Campinas.

LUEDERWALDT, H. 1919. Os manguezais de Santos. Rev. Mus. Paulista 11:309-408.

MACIEL, N.C. 1986. Desarraigamento de manguezal, através de barragem de rios e gamboas com aterro para a implantação de salina, no Município de Galinhos, Rio Grande do Norte. FEEMA, Rio de Janeiro, 23 p. (Parecer técnico da FEEMA).

MALTZ, A. \& FAERMAN, S. 1958. Moluscos comestíveis do Brasil, berbigões e mexilhões comuns do litoral paulista. An. Farm. Quím. 9(3/4):45-49.

OLSON, D.M., DINERSTEIN, E., CINTRÓN, G. \& IOLSTER, P. (eds). 1996. A conservation assessment of mangrove ecosystem of Latin América and the Caribbean. In WWF's Conservation Assessment of mangrove ecosystems of Latin America and the Caribbean Workshop. WWF, Washington, DC. USA.

PIRES-VANIN, A.M.S., CORBISIER, T.N., ARASAKI, E. \& MÖELLMANN, A.M. 1997. Composição e distribuição espaço-temporal da fauna bêntica no Canal de São Sebastião. Rel. Técn. Inst. Oceanogr. 41:29-46.

QUAST, M.P., SOLFERINI, V.N. \& AMARAL, A.C.Z. 2009. Desenvolvimento de metodologia para estudos taxonômicos moleculares em Corbula
(Bivalvia: Corbulidae). In XXI Encontro Brasileiro de Malacologia. Sociedade Brasileira de Malacolgia, Rio de Janeiro.

RODRIGUES, S.A. 1999. Filo Hemichordata. In Série Biodiversidade do Estado de São Paulo, Brasil: Síntese do conhecimento ao final do século XX, 3: Invertebrados Marinhos. (A.E. Migotto \& Tiago, C.G., eds). Programa de Pesquisa e Conservação e Uso Sustentável da Biodiversidade de São Paulo (BIOTA/FAPESP), São Paulo, SP, p. 273-276.

ROSSI-WONGTSCHOWSKI, C.L.D.B., SOARES, L.S.H. \& MUTO, E.Y. 1997. A ictiofauna demersal do Canal e da Plataforma Interna de São Sebastião. Rel. Técn. Inst. Oceanogr. 41:47-64.

SAWAYA, P. 1950. Reencontro de Balanoglossus gigas Fr. Müller no litoral brasileiro. Bolm. Inst. oceanogr. 1(1):135-138.

SAWAYA, P. 1951. Balanoglossus gigas Fr. Müller rediscovered on the Brazilian Coast. Nature 167:730.

SCHAEFFER-NOVELLI, Y. 1976. Alguns aspectos ecológicos e análise da população de Anomalocardia brasiliana (Gmelin, 1791) MolluscaBivalvia, na praia do Saco da Ribeira, Ubatuba, Estado de São Paulo. Tese de Doutorado, Universidade de São Paulo, São Paulo.

SCHAEFFER-NOVELLI, Y. 1986. Manguezais brasileiros: uma bibliografia (1614-1986). Instituto Oceanográfico, Universidade de São Paulo, Superintendência do Desenvolvimento do Litoral Paulista, São Paulo.

SCHAEFFER-NOVELLI, Y. 1991. Manguezais brasileiros: texto que sistematiza criticamente parte da produção científica. Tese de Livredocência, Universidade de São Paulo, São Paulo.

SCHAEFFER-NOVELLI, Y. 2000. Manguezal, os dispositivos legais como instrumento da conservação. In V Simpósio de Ecossistemas Brasileiros: Conservação e Duna. ACIESP, São Paulo, p. 10-17.

SEADE. 2009. Fundação Sistema Estadual de Análise de Dados. http://www. sp.gov.br/ produtos/imp/index.php (último acesso em 08/05/2009).

SPENGEL, J.W. 1893. Die Enteropneusten des Golfes von Neapel und der Angrenzenden Meeres - Abschnitte. Fauna Flora Golf. Neape. 18:1-755.

STEINER, T.M. \& AMARAL, A.C.Z. 2009. Arabella aracaensis, a new species with growth rings on its mandibles, and some remarks on the endoparasitic Labrorostratus prolificus (Polychaeta: Oenonidae) from southeastern Brazil. J. Nat. Hist. 43(41):2537-2551.

STEINER, T.M. 2005. Estudo Taxonômico da Família Onuphidae (Annelida, Polychaeta) da Costa Sudeste e Sul do Brasil. Tese de Doutorado, Universidade de São Paulo, São Paulo.

TOBIAS E SILVA, M.M. 1957. Moluscos comestíveis do Brasil. An. Farm. Quim. 8(5/6):5-13.

VANNUCCI, M. 2002. Os manguezais e nós. 2 ed., EDUSP, São Paulo.

Recebido em 28/10/09

Versão reformulada recebida em 18/01/10

Publicado em 1/02/10 
Apêndice 1. Lista das espécies de plantas e invertebrados da Baía do Araçá e adjacências, citação bibliográfica, material depositado em coleção científica e registro de novos táxons para a ciência.

Appendix 1. Species list of invertebrates and plants from Araçá Bay and adjacencies, bibliographic citation, deposited material in scientific collection and new taxa to science.

\begin{tabular}{|c|c|c|}
\hline Táxon superior Espécie & Citação bibliográfica e/ou Coleção Científica & $\begin{array}{l}\text { Localidade- } \\
\text { tipo Araçá }\end{array}$ \\
\hline CHLOROPHYTA & & - \\
\hline Bryopsis sp. & Milanelli, 2003 & - \\
\hline Caulerpa fastigiata Montagne & Milanelli, 2003; SPFUSP & - \\
\hline Caulerpa racemosa (Forsskal) J. Agardh & Duarte, 1980b; Milanelli, 2003; SPFUSP & - \\
\hline Caulerpa sertularioides (S.G. Gmelin) M.A. Howe & Milanelli, 2003; SPFUSP & - \\
\hline Cladophora fascicularis (Mertens ex C. Agardh) Kützing & SPFUSP (como Cladophora vagabunda) & - \\
\hline Cladophoropsis sp. & SPFUSP & - \\
\hline Codium decorticatum (Woodward) M.A. Howe & Milanelli, 2003 & - \\
\hline Codium intertextum F.S. Collins \& Hervey & Migotto, 1993; Milanelli, 2003 & - \\
\hline Codium sp. & Milanelli, 2003 & - \\
\hline Codium taylorii P.C. Silva & Milanelli, 2003 & - \\
\hline Derbesia marina (Lyngbye) Solier & SPFUSP & - \\
\hline Enteromorpha chaetomorphoides Børgesen & SPFUSP (como Ulva chaetomorphoides) & - \\
\hline Enteromorpha clathrata (Roth) Greville & SPFUSP (como Ulva clathrata) & - \\
\hline Enteromorpha lingulata J. Agardh & SPFUSP (como Ulva flexuosa) & - \\
\hline Enteromorpha linza (Linnaeus) J. Agardh & SPFUSP (como Ulva linza) & - \\
\hline Enteromorpha sp. & Milanelli, 2003 & - \\
\hline Monostroma sp. & HIBSP & - \\
\hline $\begin{array}{l}\text { Pedobesia ryukyuensis (Yamada \& T.Tanaka) Kobara \& } \\
\text { Chihara }\end{array}$ & SPFUSP & - \\
\hline Rhizoclonium sp. & SPFUSP & - \\
\hline Ulva fasciata Delile & Duarte, 1980b; SPFUSP & - \\
\hline Ulva lactuca Linnaeus & SPFUSP & - \\
\hline Ulva sp. & Milanelli, 2003 & - \\
\hline Ulvaria oxysperma (Kützing) Bliding & SPFUSP & - \\
\hline RHODOPHYTA & & - \\
\hline Acanthophora spicifera (M. Vahl) Børgesen & Duarte, 1980b; Migotto, 1993; Milanelli, 2003; SPFUSP & - \\
\hline Amphiroa sp. & Milanelli, 2003 & - \\
\hline Bostrychia binderi Harvey & Milanelli, 2003 & - \\
\hline Bostrychia radicans (Montagne) Montagne & Milanelli, 2003; SPFUSP & - \\
\hline Callithamnion felipponei $\mathrm{M}$. Howe & Milanelli, 2003 & - \\
\hline Callithamnion uruguayense W.R. Taylor & Milanelli, 2003 & - \\
\hline Caloglossa leprieurii (Montagne) G. Martens & SPFUSP & - \\
\hline Centroceras clavulatum (C. Agardh) Montagne & Milanelli, 2003; SPFUSP & - \\
\hline Ceramium brevizonatum H.E. Petersen & SPFUSP & - \\
\hline Ceramium sp. & Milanelli, 2003 & - \\
\hline Chondracanthus acicularis (Roth) Fredericq & Migotto, 1993; Milanelli, 2003 (como Gigartina acicularis) & - \\
\hline Chondracanthus teedei (Mertens ex Roth) Kützing & Migotto, 1993 (como Gigartina teedei) & - \\
\hline Corallina officinalis Linnaeus & Milanelli, 2003 & - \\
\hline Dasya brasiliensis E.C. Oliveira \& Braga & Milanelli, 2003 & - \\
\hline Dawsoniocolax sp. & SPFUSP & - \\
\hline Falkenbergia hillebrandii (Bornet) Falkenberg & Milanelli, 2003 & - \\
\hline Galaxaura frutescens Kjellman & Milanelli, 2003 & - \\
\hline
\end{tabular}

HIBSP - Herbário Científico Maria Eneyda P. K. Fidalgo do Instituto de Botânica de São Paulo

MNRJ - Museu Nacional do Rio de Janeiro

MZUSP - Museu de Zoologia da Universidade de São Paulo

SPFUSP - Coleção de Algas do Departamento de Botânica da Universidade de São Paulo

USNM - National Museum of Natural History, Smithsonian Institution, USA 
Apêndice 1. Continuação...

\begin{tabular}{|c|c|c|}
\hline Táxon superior Espécie & Citação bibliográfica e/ou Coleção Científica & $\begin{array}{l}\text { Localidade- } \\
\text { tipo Araçá }\end{array}$ \\
\hline Galaxaura marginata (Ellis \& Solander) J.V. Lamouroux & Milanelli, 2003 & - \\
\hline Galaxaura sp. & Migotto, 1993; Milanelli, 2003 & - \\
\hline Gelidium pusillum (Stackhouse) Le Jolis & Milanelli, 2003 & - \\
\hline Gelidium sp. & Milanelli, 2003; SPFUSP & - \\
\hline Gracilaria caudata J. Agardh & SPFUSP & - \\
\hline Gracilaria cervicornis (Turner) J. Agardh & SPFUSP & - \\
\hline Gracilaria sp. & Milanelli, 2003 & - \\
\hline $\begin{array}{l}\text { Gracilariopsis tenuifrons (C.J. Bird \& E.C. Oliveira) } \\
\text { Fredericq \& Hommersand }\end{array}$ & SPFUSP; Plastino, 1991 & - \\
\hline Grateloupia sp. & SPFUSP & - \\
\hline Gymnogongrus griffithsiae (Turner) Martius & SPFUSP & - \\
\hline Herposiphonia sp. & Milanelli, 2003 & - \\
\hline Heterosiphonia gibbesii (Harvey) Falkenberg & SPFUSP & - \\
\hline Hypnea cervicornis J. Agardh & Milanelli, 2003; SPFUSP & - \\
\hline Hypnea cornuta (Kützing) J. Agardh, 1851 & SPFUSP & - \\
\hline Hypnea musciformis (Wulfen) J.V. Lamouroux & SPFUSP & - \\
\hline Hypnea sp. & Milanelli, 2003 & - \\
\hline Hypnea spinella (C. Agardh) Kützing & SPFUSP & - \\
\hline Jania capillacea Harvey & Milanelli, 2003 & - \\
\hline Jania sp. & Duarte, 1980b; Milanelli, 2003 & - \\
\hline Laurencia papillosa (C. Agardh) Greville & Duarte, 1980b; & - \\
\hline Laurencia sp. & Milanelli, 2003 & - \\
\hline Polysiphonia sp. & SPFUSP & - \\
\hline Porphyra atropurpurea (Olivi) De Toni & Milanelli, 2003 & - \\
\hline Porphyra spiralis E.C. Oliveira \& Coll & SPFUSP & - \\
\hline Pterocladia pinnata (Hudson) Papenfuss & Duarte, 1980b; & - \\
\hline Solieria sp. & SPFUSP & - \\
\hline Spyridia filamentosa (Wulfen) Harvey & SPFUSP & - \\
\hline Taenioma perpusillum (J. Agardh) J. Agardh & Milanelli, 2003 & - \\
\hline Wrangelia argus (Montagne) Montagne & Milanelli, 2003 & - \\
\hline PHAEOPHYTA & & - \\
\hline $\begin{array}{l}\text { Asteronema breviarticulatum (J. Agardh) Ouriques \& } \\
\text { Bouzon }\end{array}$ & Milanelli, 2003 (como Ectocarpus breviarticulatus) & - \\
\hline Colpomenia sinuosa (Mertens ex Roth) Derbès \& Solier & Milanelli, 2003 & - \\
\hline Dictyopteris delicatula J.V. Lamouroux & Milanelli, 2003 & - \\
\hline Dictyopteris sp. & Migotto, 1993; Milanelli, 2003; SPFUSP & - \\
\hline Dictyota cervicornis Kützing & Milanelli, 2003 & - \\
\hline Dictyota dichotoma (Hudson) J.V. Lamouroux & Milanelli, 2003; SPFUSP & - \\
\hline Dictyota sp. & Migotto, 1993; Milanelli, 2003 & - \\
\hline Padina gymnospora (Kützing) Sonder & Milanelli, 2003; SPFUSP & - \\
\hline Padina sp. & Milanelli, 2003 & - \\
\hline Sargassum cymosum C. Agardh & Duarte \& Nalesso, 1996 & - \\
\hline Sargassum furcatum Kützing & SPFUSP & - \\
\hline Sargassum sp. & Migotto, 1993; Milanelli, 2003 & - \\
\hline Sargassum vulgare C. Agardh & Milanelli, 2003 & - \\
\hline \multicolumn{3}{|c|}{ HIBSP - Herbário Científico Maria Eneyda P. K. Fidalgo do Instituto de Botânica de São Paulo } \\
\hline \multicolumn{3}{|c|}{ MNRJ - Museu Nacional do Rio de Janeiro } \\
\hline \multicolumn{3}{|l|}{ MZUSP - Museu de Zoologia da Universidade de São Paulo } \\
\hline \multicolumn{3}{|c|}{ SPFUSP - Coleção de Algas do Departamento de Botânica da Universidade de São Paulo } \\
\hline JSNM - National Museum of Natural History, Smithsonian Instituti & tion, USA & \\
\hline
\end{tabular}


Apêndice 1. Continuação...

\begin{tabular}{|c|c|c|}
\hline Táxon superior Espécie & Citação bibliográfica e/ou Coleção Científica & $\begin{array}{c}\text { Localidade- } \\
\text { tipo Araçá }\end{array}$ \\
\hline MAGNOLIOPHYTA & & - \\
\hline Avicennia schaeuriana Stapf \& Leechman & Lamparelli et al. 1998 & - \\
\hline Halodule emarginata den Hartog & den Hartog, 1970 & $\mathrm{X}$ \\
\hline Laguncularia racemosa (L.) Gaerten & Lamparelli et al. 1998 & - \\
\hline Rhizophora mangle Linnaeus & Castelo-Branco et al. 1996 & - \\
\hline CILIOPHORA & & - \\
\hline Blepharisma clarissimum Kahl, 1928 & Kattar, 1966 & - \\
\hline Condylostoma remanei Spiegel, 1926 & Kattar, 1970 & - \\
\hline Frontonia marina Fabre-Domergue, 1891 & Kattar, 1966 & - \\
\hline Geleia orbis Fauré-Fremiet, 1950 & Kattar, 1970 & - \\
\hline $\begin{array}{l}\text { Psammocephalus lithophora (Fauré-Fremiet, 1954) } \\
\text { Wicklow, } 1982\end{array}$ & Kattar, 1970 (como Amphisiella lithophora); Wicklow, 1982 & - \\
\hline Strongylidium caudatum Kahl, 1935 & Kattar, 1966, 1970 (como Urostrongylum caudatum) & - \\
\hline PORIFERA & & - \\
\hline Cliona aff. celata Grant, 1826 & MNRJ & - \\
\hline Halichondria sulfurea Carvalho \& Hajdu, 2001 & Carvalho \& Hajdu, 2001; MNRJ & $\mathrm{X}$ \\
\hline Haliclona melana Muricy \& Ribeiro, 1999 & MNRJ & - \\
\hline Haliclona sp. & Milanelli, 2003 & - \\
\hline Hymeniacidon heliophila (Parker, 1910) & MNRJ & - \\
\hline Hymeniacidon? sp. & MNRJ & - \\
\hline Hyrtios / Psammopemma & MNRJ & - \\
\hline Microcionidae sp. & MNRJ & - \\
\hline Mycale aff. americana van Soest, 1984 & MNRJ & - \\
\hline Mycale angulosa (Duchassaing \& Michelotti, 1864) & $\begin{array}{l}\text { Morgado \& Duarte, 1979; Duarte, 1980a,b, 1982; Duarte \& } \\
\text { Morgado, 1983;Duarte \& Nalesso, } 1996 \text { (como Zygomicale } \\
\text { parishi, ver Ribeiro et al. 2003) }\end{array}$ & - \\
\hline Oceanapia nodosa (George \& Wilson, 1919) & MNRJ & - \\
\hline Tedania ignis (Duchassaing \& Michelotti, 1864) & Milanelli, 2003 & - \\
\hline CNIDARIA, ANTHOZOA & & - \\
\hline Anemonia sargassensis Hargitt, 1908 & MZUSP & - \\
\hline Bunodosoma caissarum Corrêa, 1964 & Milanelli, 2003; MZUSP & - \\
\hline Carijoa riisei (Duchassaing \& Michelotti, 1860) & Migotto, 1993 & - \\
\hline Leptogorgia setacea (Pallas, 1766) & MZUSP & - \\
\hline Renilla sp. & Soares, 1970 & - \\
\hline CNIDARIA, HYDROZOA & & - \\
\hline Aglaophenia latecarinata Allman, 1877 & Migotto, 1993, 1996; Milanelli, 2003 & - \\
\hline Bimeria vestita Wright, 1859 & Migotto, 1993, 1996 & - \\
\hline Cladocoryne floccosa Rotch, 1871 & Migotto, 1993, 1996 & - \\
\hline Clytia hemisphaerica (Linnaeus, 1767) & Migotto, 1993, 1996 & - \\
\hline Clytia linearis (Thornely, 1899) & Migotto, 1993; & - \\
\hline Diphasia tropica Nutting, 1904 & Migotto, 1993, 1996 & - \\
\hline Dipurena reesi Vannucci, 1956 & Moreira et al. 1978 & - \\
\hline Dynamena crisioides Lamouroux, 1824 & Migotto, 1993, 1996 & - \\
\hline Dynamena disticha (Bosc, 1802) & Migotto, 1993, 1996 & - \\
\hline Dynamena quadridentata (Ellis \& Solander, 1786) & Migotto, 1993, 1996 & - \\
\hline
\end{tabular}

HIBSP - Herbário Científico Maria Eneyda P. K. Fidalgo do Instituto de Botânica de São Paulo

MNRJ - Museu Nacional do Rio de Janeiro

MZUSP - Museu de Zoologia da Universidade de São Paulo

SPFUSP - Coleção de Algas do Departamento de Botânica da Universidade de São Paulo

USNM - National Museum of Natural History, Smithsonian Institution, USA 
Apêndice 1. Continuação...

\begin{tabular}{|c|c|c|}
\hline Táxon superior Espécie & Citação bibliográfica e/ou Coleção Científica & $\begin{array}{l}\text { Localidade- } \\
\text { tipo Araçá }\end{array}$ \\
\hline Eudendrium carneum Clarke, 1882 & Marques, 1993 & - \\
\hline Halecium tenellum Hincks, 1861 & Migotto, 1993, 1996 & - \\
\hline Halopteris alternata (Nutting, 1900) & Migotto, 1993, 1996 (como Halopteris diaphana) & - \\
\hline Hebella scandens (Bale, 1888) & Migotto, 1993, 1996 & - \\
\hline Lytocarpia tridentata Versluys, 1899 & Migotto, 1993, 1996 & - \\
\hline Monotheca margaretta Nutting, 1900 & Migotto, 1993, 1996 & - \\
\hline Nemalecium lighti (Hargitt, 1924) & $\begin{array}{l}\text { Migotto, } 1993 \text { (como Kirchenpaueria halecioides); Migotto, } \\
1996\end{array}$ & - \\
\hline Obelia bidentata Clark, 1875 & Migotto, 1993, 1996 & - \\
\hline Obelia dichotoma (Linnaeus, 1758) & Migotto, 1993, 1996 & - \\
\hline Orthopyxis sargassicola (Nutting, 1915) & Migotto, 1993, 1996 & - \\
\hline Plumularia sp. & Milanelli, 2003 & - \\
\hline Sertularella conica Allman, 1877 & Migotto, 1993, 1996 & - \\
\hline Sertularia distans Lamouroux 1816 & Migotto, 1993, 1996 & - \\
\hline Sertularia marginata (Kirchenpauer, 1864) & Migotto, 1993, 1996, 1998 & - \\
\hline Sertularia turbinata (Lamouroux 1816) & Migotto, 1993, 1996; Milanelli, 2003 & - \\
\hline Stylactis hooperi Sigerfoos 1899 & $\begin{array}{l}\text { Moreira et al. 1979; Nipper-Buscariolli \& Moreira, 1982, 1983; } \\
\text { Nipper-Buscariolli, 1983; Migotto, } 1996\end{array}$ & - \\
\hline Ventromma halecioides (Alder, 1859) & $\begin{array}{l}\text { Moreira \& Nipper, 1978; Nipper-Buscariolli \& Moreira, 1982, } \\
\text { 1983; Nipper-Buscariolli, 1983; Migotto, 1993, } 1996\end{array}$ & - \\
\hline NEMERTEA & & - \\
\hline Baseodiscus curtus (Hubrechet, 1879) & Nalesso et al. 1995; Duarte \& Nalesso, 1996 & - \\
\hline MOLLUSCA, POLYPLACOPHORA & & - \\
\hline Acanthochitona sp. & Milanelli, 2003 & - \\
\hline Calloplax janeirensis (Gray, 1828) & Migotto et al. 1993 & - \\
\hline Chaetopleura angulata (Spengler, 1797) & Migotto et al. 1993 & - \\
\hline Chaetopleura asperrima (Gould, 1852) & Migotto et al. 1993 & - \\
\hline Ischnochiton striolatus (Gray, 1828) & Migotto et al. 1993; Nalesso et al. 1995 & - \\
\hline MOLLUSCA, GASTROPODA & & - \\
\hline Anachis brasiliana E. von Martens, 1897 & Duarte, 1980b; & - \\
\hline Anachis lyrata (Sowerby, 1832) & $\begin{array}{l}\text { Migotto et al. 1993; Nalesso et al. 1995; Duarte \& Nalesso, } \\
1996\end{array}$ & - \\
\hline Anachis obesa (C.B.Adams, 1845) & Duarte, $1980 \mathrm{~b}$ & - \\
\hline Anachis sparsa (Reeve, 1859) & Duarte, $1980 \mathrm{~b}$ & - \\
\hline Anachis veleda (Duclos, 1846) & Duarte, $1980 \mathrm{~b}$ & - \\
\hline Aplysia brasiliana Rang, 1828 & $\begin{array}{l}\text { Leahy, 1970, 1975; Sawaya \& Leahy, 1971; Acevedo, 1974, } \\
\text { Scemes et al. 1991; Migotto et al. 1993; Nalesso et al. 1995; } \\
\text { Polatto et al. } 2002\end{array}$ & - \\
\hline Aplysia juliana Quoy \& Gaimard, 1832 & Migotto et al. 1993 & - \\
\hline Astraea olfersii (Philippi, 1846) & Migotto et al. 1993; Milanelli, 2003 (como Astraea phoebia) & - \\
\hline Berthella tupala Marcus, 1957 & Nalesso et al. 1995 & - \\
\hline Bitium varium Pfeiffer, 1840 & $\begin{array}{l}\text { Duarte, 1980b; Migotto et al. 1993; Belúcio, 1995; Nalesso } \\
\text { et al. 1995; Duarte \& Nalesso, } 1996\end{array}$ & - \\
\hline Bulla striata Bruguière, 1792 & $\begin{array}{l}\text { Duarte, 1980b; Ditadi, 1982b; Migotto et al. 1993; Belúcio, } \\
\text { 1995; Duarte \& Nalesso, 1996; Dworschak \& Rodrigues, 1997; } \\
\text { Arruda \& Amaral, } 2003\end{array}$ & - \\
\hline
\end{tabular}

HIBSP - Herbário Científico Maria Eneyda P. K. Fidalgo do Instituto de Botânica de São Paulo

MNRJ - Museu Nacional do Rio de Janeiro

MZUSP - Museu de Zoologia da Universidade de São Paulo

SPFUSP - Coleção de Algas do Departamento de Botânica da Universidade de São Paulo

USNM - National Museum of Natural History, Smithsonian Institution, USA 
Apêndice 1. Continuação...

\begin{tabular}{|c|c|c|}
\hline Táxon superior Espécie & Citação bibliográfica e/ou Coleção Científica & $\begin{array}{l}\text { Localidade- } \\
\text { tipo Araçá }\end{array}$ \\
\hline Bursatella leachi Blainville, 1817 & Migotto et al. 1993 & - \\
\hline Caecum pulchellum Stimpson, 1851 & MZUSP & - \\
\hline Caliphylla mediterrânea Costa, 1867 & Migotto et al. 1993 & - \\
\hline Cerithiopsis emersoni (C.B. Adams, 1838) & Migotto et al. 1993; Belúcio, 1995; Arruda \& Amaral, 2003 & - \\
\hline Cerithium atratum (Born, 1778) & $\begin{array}{l}\text { Moreira et al. 1978; Moreira et al. 1979; Nipper-Buscariolli \& } \\
\text { Moreira, 1982, 1983; Nipper-Buscariolli, 1983; Migotto, 1993; } \\
\text { Migotto et al. 1993; Belúcio, 1995; Arruda, 2000; Amaral et al. } \\
\text { 2003; Arruda et al. 2003; Milanelli, 2003; Denadai et al. 2005; } \\
\text { Turra \& Leite, 2005; Padovanni, } 2009\end{array}$ & - \\
\hline Cerithium muscarum Say, 1832 & Dworschak \& Rodrigues, 1997 & - \\
\hline Cerithium sp. & Ditadi, $1982 b$ & - \\
\hline Chaetopleura aspérrima (Gould, 1852) & Nalesso et al. 1995 & - \\
\hline Chromodoris neona (Er. Marcus, 1955) & Migotto et al. 1993 & - \\
\hline Collisella subrugosa (d’Orbigny, 1846) & Migotto et al. 1993; Milanelli, 2003 & - \\
\hline Columbella mercatoria (Linnaeus, 1758) & Migotto et al. 1993; Nalesso et al. 1995 & - \\
\hline Costoanachis catenata (Sowerby, 1844) & Migotto et al. 1993 & - \\
\hline Costoanachis sertulariarum (d'Orbigny, 1839) & $\begin{array}{l}\text { Migotto et al. 1993; Duarte \& Nalesso, 1996; Morgado \& } \\
\text { Tanaka, 2001; Milanelli, } 2003\end{array}$ & - \\
\hline Costoanachis sparsa (Reeve, L.A., 1859) & $\begin{array}{l}\text { Nalesso et al. 1995; Duarte \& Nalesso, 1996; Morgado \& } \\
\text { Tanaka, } 2001\end{array}$ & - \\
\hline Bostrycapulus odites Collin, 2005 & $\begin{array}{l}\text { Migotto et al. 1993; Morgado \& Tanaka, } 2001 \text { (como Crepidula } \\
\text { aculeata) }\end{array}$ & - \\
\hline Crepidula intratesta Simone, 2006 & $\begin{array}{l}\text { Morgado, 1980b; Migotto et al. 1993; Morgado \& Tanaka, } 2001 \\
\text { (como Crepidula plana) }\end{array}$ & - \\
\hline Cylichnella bidentata (d'Orbigny, 1841) & Migotto et al. 1993 & - \\
\hline Cymatium parthenopeum (Von Salis, 1793) & Ditadi, 1982b; Migotto et al. 1993 & - \\
\hline Cypraea zebra Linnaeus, 1758 & Milanelli, 2003 & - \\
\hline Dendrodoris sp. & Migotto et al. 1993 & - \\
\hline Diodora patagonica (d'Orbigny, 1847) & Migotto et al. 1993; MZUSP & - \\
\hline Doris bovena Er. Marcus, 1955 & Migotto et al. 1993 & - \\
\hline Doris verrucosa Linnaeus, 1758 & $\begin{array}{l}\text { Duarte, 1980b; Migotto et al. 1993; Nalesso et al. 1995; Duarte } \\
\text { \& Nalesso, 1996; Milanelli, } 2003\end{array}$ & - \\
\hline Epitonium sp. & Belúcio, 1995 & - \\
\hline Espererato maugeriae (Gray, 1832) & $\begin{array}{l}\text { Duarte, 1980b; Duarte \& Nalesso, } 1996 \text { (como Erato } \\
\text { maugeriae) }\end{array}$ & - \\
\hline Ercolania sp. & MZUSP & - \\
\hline Eulima sp. & MZUSP & - \\
\hline Finella dubia (d'Orbigny, 1840) & Migotto et al. 1993 & - \\
\hline Fissurella clenchi Farfante, 1943 & Migotto et al. 1993; Milanelli, 2003 & - \\
\hline Haminoea elegans (Gray, 1825) & Migotto et al. 1993 & - \\
\hline Lamellaria mopsicolor Ev. Marcus, 1958 & $\begin{array}{l}\text { Duarte, 1980b; Duarte \& Nalesso, } 1996 \text { (como Lamellaria } \\
\text { perspicua) }\end{array}$ & - \\
\hline Leucozonia nassa (Gmelin, 1791) & Milanelli, 2003 & - \\
\hline Littorina angulifera (Lamarck, 1822) & Migotto et al. 1993 & - \\
\hline Littorina flava King \& Broderip, 1832 & Migotto et al. 1993; Milanelli, 2003 & - \\
\hline Lophodoris scala Er. Marcus \& Ev. Marcus, 1970 & Ditadi, 1970a; Marcus \& Marcus 1970; Anker et al. 2005 & $\mathrm{X}$ \\
\hline Mangelia quadrilineata (C. B. Adams, 1850) & Belúcio, 1995 & - \\
\hline
\end{tabular}

HIBSP - Herbário Científico Maria Eneyda P. K. Fidalgo do Instituto de Botânica de São Paulo

MNRJ - Museu Nacional do Rio de Janeiro

MZUSP - Museu de Zoologia da Universidade de São Paulo

SPFUSP - Coleção de Algas do Departamento de Botânica da Universidade de São Paulo

USNM - National Museum of Natural History, Smithsonian Institution, USA 
Apêndice 1. Continuação...

\begin{tabular}{|c|c|c|}
\hline Táxon superior Espécie & Citação bibliográfica e/ou Coleção Científica & $\begin{array}{l}\text { Localidade- } \\
\text { tipo Araçá }\end{array}$ \\
\hline Mitrella argus Orbigny, 1842 & $\begin{array}{l}\text { Migotto et al. 1993; Nalesso et al. 1995; Morgado \& Tanaka, } \\
2001\end{array}$ & - \\
\hline Mitrella dichroa (Sowerby, I., 1844) & Morgado, 1980b; Duarte \& Nalesso, 1996 & - \\
\hline Mitrela lunata (Say 1826) & Duarte, $1980 b$ & - \\
\hline Modulus modulus (Linnaeus, 1758) & Migotto et al. 1993 & - \\
\hline Morula nodulosa (C.B. Adams, 1845) & $\begin{array}{l}\text { Migotto et al. 1993; Nalesso et al. 1995; Morgado \& Tanaka, } \\
\text { 2001; Milanelli, 2003; Turra \& Leite, } 2005\end{array}$ & - \\
\hline Nassarius vibex (Say, 1822) & $\begin{array}{l}\text { Belúcio \& Morgado, 1991, 1995; Migotto et al. 1993; Belúcio, } \\
\text { 1995; Arruda, 2000; Arruda \& Amaral, 2003; Arruda et al. } \\
\text { 2003; Denadai et al. 2005; Padovanni, } 2009\end{array}$ & - \\
\hline Nassarius sp. & Morgado \& Tanaka, 2001 & - \\
\hline Natica cayennensis Récluz, 1850 & Migotto et al. 1993 & - \\
\hline Neritina virginea (Linnaeus, 1758) & Migotto et al. 1993; Padovanni, 2009 & - \\
\hline Nitidella dichroa (Sowerby I, 1844) & Duarte, 1980b; Morgado, 1980b & - \\
\hline Nodilittorina lineolata (d'Orbigny, 1840) & Migotto et al. 1993; Milanelli, 2003 & - \\
\hline Odostomia sp. & Migotto et al. 1993 & - \\
\hline Olivella minuta (Link, 1807) & $\begin{array}{l}\text { Amaral et al. 1990; Migotto et al. 1993; Belúcio, 1995; Belúcio } \\
\text { \& Morgado; 1995; Arruda, 2000; Amaral et al. 2003; Arruda } \\
\text { \& Amaral, 1998, 1999, 2003; Arruda et al. 1997a,b; 2003; } \\
\text { Denadai et al. 2005; Padovanni, } 2009\end{array}$ & - \\
\hline Onchidella indolens (Couthouy, 1852) & Migotto et al. 1993; Milanelli, 2003 & - \\
\hline Parvanachis obesa (Adams, 1845) & Migotto et al. 1993; Duarte \& Nalesso, 1996 & - \\
\hline Pisania auritula (Link, 1807) & Milanelli, 2003 & - \\
\hline Pisania pusio (Linnaeus, 1758), & Milanelli, 2003 & - \\
\hline Polinices hepaticus (Röding, 1798) & Migotto et al. 1993 & - \\
\hline Retusa candei (d'Orbigny, 1841) & Belúcio, 1995 & - \\
\hline Rissoina catesbyana (d'Orbigny, 1842) & $\begin{array}{l}\text { Migotto et al. 1993; Nalesso et al. 1995; Duarte \& Nalesso, } \\
1996\end{array}$ & - \\
\hline Rissoina chesnelii (Michaud, 1830) & Duarte, $1980 b$ & - \\
\hline Seila adamsi (H.C. Lea, 1845) & $\begin{array}{l}\text { Duarte, 1980b; Nalesso et al. 1995; Duarte \& Nalesso, 1996; } \\
\text { Morgado \& Tanaka, } 2001\end{array}$ & - \\
\hline Siphonaria hispida E.A. Smith, 1890 & Migotto et al. 1993; Milanelli, 2003 & - \\
\hline Siratus tenuivaricosus (Dautzenberg, 1927) & MZUSP & - \\
\hline Solariella sp. & Morgado \& Tanaka, 2001 & - \\
\hline Spurilla neapolitana (delle Chiaje, 1844) & Migotto et al. 1993; Milanelli, 2003 & - \\
\hline Tegula viridula (Gmelin, 1791) & $\begin{array}{l}\text { Migotto et al. 1993; Milanelli, 2003; Denadai et al. 2005; Turra } \\
\text { \& Leite, } 2005\end{array}$ & - \\
\hline Thais haemastoma (Linnaeus, 1767) & $\begin{array}{l}\text { Magalhães, 1987a; Magalhães, 1988; Migotto et al. 1993; } \\
\text { Nalesso et al. 1995; Turra \& Leite, } 2005 \text { (como Stramonita } \\
\text { haemastoma) }\end{array}$ & - \\
\hline Thecacera pennigera (Montagu, 1815) & MZUSP & - \\
\hline Trapania sp. & MZUSP & - \\
\hline Tricolia affinis (C.B. Adams, 1850) & Migotto et al. 1993; Nalesso et al. 1995 & - \\
\hline MOLLUSCA, BIVALVIA & & - \\
\hline Abra aequalis (Say, 1822) & Padovanni, 2009 & - \\
\hline \multicolumn{3}{|c|}{ HIBSP - Herbário Científico Maria Eneyda P. K. Fidalgo do Instituto de Botânica de São Paulo } \\
\hline \multicolumn{3}{|l|}{ MNRJ - Museu Nacional do Rio de Janeiro } \\
\hline \multicolumn{3}{|c|}{ MZUSP - Museu de Zoologia da Universidade de São Paulo } \\
\hline \multicolumn{3}{|c|}{ SPFUSP - Coleção de Algas do Departamento de Botânica da Universidade de São Paulo } \\
\hline USNM - National Museum of Natural History, Smit & tion, USA & \\
\hline
\end{tabular}

USNM - National Museum of Natural History, Smithsonian Institution, USA 
Apêndice 1. Continuação...

\begin{tabular}{|c|c|c|}
\hline Táxon superior Espécie & Citação bibliográfica e/ou Coleção Científica & $\begin{array}{l}\text { Localidade- } \\
\text { tipo Araçá }\end{array}$ \\
\hline Abra lioica (Dall, 1881) & $\begin{array}{l}\text { Migotto et al. 1993; Belúcio, 1992, 1995; Belúcio \& Morgado, } \\
\text { 1994, 1995; Arruda \& Amaral, 2003; Arruda et al. 2003; } \\
\text { Denadai et al. } 2005\end{array}$ & - \\
\hline Anadara notabilis (Röding, 1798) & Ditadi, $1982 b$ & - \\
\hline Anomalocardia brasiliana (Gmelin, 1791) & $\begin{array}{l}\text { Leonel, 1981; Ditadi, 1982b; Montouchet, 1988; Belúcio \& } \\
\text { Morgado, 1991, 1994, 1995; Migotto et al. 1993; Belúcio, } \\
\text { 1992, 1995; Dworschak \& Rodrigues, 1997; Arruda \& Amaral, } \\
\text { 1998, 1999, 2003; Arruda, 2000; Amaral et al. 2003; Arruda } \\
\text { et al. 2003; Denadai et al. 2005, 2006; Padovanni, 2009; Corte } \\
\text { (em andamento) }\end{array}$ & - \\
\hline Anomia simplex d'Orbigny, 1853 & Migotto et al. 1993 (como Anomia ephipium) & - \\
\hline Arca imbricata Bruguière, 1789 & $\begin{array}{l}\text { Nalesso et al. 1995; Migotto et al. 1993; Morgado \& Tanaka, } \\
\text { 2001; Milanelli, } 2003\end{array}$ & - \\
\hline Arcopsis adamsi (Dall, 1886) & Migotto et al. 1993 & - \\
\hline Bankia campanellata Moll \& Roch, 1931 & Migotto et al. 1993 & - \\
\hline Bankia fimbriatula Moll \& Roch, 1931 & Migotto et al. 1993 & - \\
\hline Bankia gouldi (Bartsch, 1908) & Migotto et al. 1993 & - \\
\hline Bankia rochi Moll, 1931 & Migotto et al. 1993 & - \\
\hline Barbatia candida (Helbling, 1779) & Migotto et al. 1993 & - \\
\hline Brachidontes darwinianus (d'Orbigny, 1842) & Migotto et al. 1993 & - \\
\hline Brachidontes solisianus (d'Orbigny, 1842) & Migotto et al. 1993 & - \\
\hline Brachidontes sp. & Milanelli, 2003 & - \\
\hline Chama congregata Conrad, 1833 & Migotto et al. 1993; Milanelli, 2003 & - \\
\hline Chione cancellata (Linnaeus, 1767) & $\begin{array}{l}\text { Arruda, 2000; Arruda \& Amaral, 2003; Arruda et al. 2003; } \\
\text { Denadai et al. 2005; Denadai et al. 2006; Padovanni, } 2009\end{array}$ & - \\
\hline Chione intapurpurea (Conrad, 1849) & Migotto et al. 1993 & - \\
\hline Chione latilirata (Conrad, 1841) & Migotto et al. 1993 & - \\
\hline Chione paphia (Linnaeus, 1767) & Duarte, 1980b; Duarte \& Nalesso, 1996 & - \\
\hline Chione subrostrata (Lamarck, 1818) & Belúcio, 1995 & - \\
\hline Chione sp. & Ditadi, 1982b & - \\
\hline Chlamys tehuelchus (d'Orbigny, 1842) & Duarte, 1980b; Nalesso et al. 1995; Duarte \& Nalesso, 1996 & - \\
\hline Codakia costata (Orbigny, 1842) & MZUSP & - \\
\hline Codakia pectinella (C.B. Adams, 1852) & Belúcio, 1995 & - \\
\hline Compsomyax subdiaphana (Carpenter, 1864) & Belúcio, 1995 & - \\
\hline Cooperella atlantica Rehder, 1943 & Belúcio, 1995; Duarte \& Nalesso, 1996 & - \\
\hline Corbula caribaea d’Orbigny, 1853 & $\begin{array}{l}\text { Montouchet, 1988; Belúcio \& Morgado, 1991, 1994, 1995; } \\
\text { Duarte, 1980b; Belúcio, 1992, 1995; Arasaki, 1997; Arruda, } \\
\text { 2000; Arruda et al. 2003; Denadai et al. 2005; Padovanni, } \\
2009\end{array}$ & - \\
\hline Corbula cubaniana d'Orbigny, 1853 & Montouchet, 1988; Belúcio \& Morgado, 1991; Belúcio, 1995 & - \\
\hline Corbula sp.1 & Denadai et al. 2005 & - \\
\hline Corbula sp. & Migotto et al. 1993; Belúcio, 1995; Arruda et al. 2003 & - \\
\hline Crassostrea rhizophorae (Guilding, 1828) & Migotto et al. 1993; Milanelli, 2003 & - \\
\hline Crassostrea sp. & Ditadi, $1982 b$ & - \\
\hline Dendropoma sp. & Milanelli, 2003 & - \\
\hline Diplodonta portesiana (d'Orbigny, 1842) & Belúcio, 1995 & - \\
\hline
\end{tabular}

HIBSP - Herbário Científico Maria Eneyda P. K. Fidalgo do Instituto de Botânica de São Paulo

MNRJ - Museu Nacional do Rio de Janeiro

MZUSP - Museu de Zoologia da Universidade de São Paulo

SPFUSP - Coleção de Algas do Departamento de Botânica da Universidade de São Paulo

USNM - National Museum of Natural History, Smithsonian Institution, USA 
Apêndice 1. Continuação...

\begin{tabular}{|c|c|c|}
\hline Táxon superior Espécie & Citação bibliográfica e/ou Coleção Científica & $\begin{array}{l}\text { Localidade- } \\
\text { tipo Araçá }\end{array}$ \\
\hline Diplodonta punctata Say, 1822 & $\begin{array}{l}\text { Migotto et al. 1993; Belúcio, 1995; Arruda, 2000; Ar- } \\
\text { ruda \& Amaral, 2003; Arruda et al. 2003; Denadai et al. 2005; } \\
\text { MZUSP }\end{array}$ & - \\
\hline Donax gemmula Morrison, 1971 & $\begin{array}{l}\text { Belúcio, 1995; Arruda, 2000; Arruda et al. 2003; Denadai } \\
\text { et al. } 2005\end{array}$ & - \\
\hline Dosinia concentrica (Born, 1778) & Ditadi, $1982 b$ & - \\
\hline Ervilia concentrica (Holmes, 1858$)$ & Belúcio, 1995 & - \\
\hline Ervilia nitens (Montagu, 1808) & Belúcio, 1995 & - \\
\hline Gouldia cerina (C.B.Adams, 1845) & Duarte, 1980b; Belúcio, 1995; Duarte \& Nalesso, 1996 & - \\
\hline Hiatella solida (Sowerby I, 1834) & $\begin{array}{l}\text { Duarte, 1980b; Morgado, 1980b, 1985; Migotto et al. 1993; } \\
\text { Nalesso et al. 1995; Duarte \& Nalesso, 1996; Morgado \& } \\
\text { Tanaka, } 2001 \text { (como Hiatella arctica) }\end{array}$ & - \\
\hline Iphigenia brasiliana (Lamarck, 1818) & Denadai et al. 2005 & - \\
\hline Leptopecten bavayi (Dautzenberg, 1900) & Migotto et al. 1993 & - \\
\hline Lioberus castaneus (Say, 1822) & Belúcio, 1995 & - \\
\hline Lithophaga bisulcata (Orbigny, 1842) & Morgado, 1980b, 1985; Morgado \& Tanaka, 2001 & - \\
\hline Lucina pectinata (Gmelin, 1791) & $\begin{array}{l}\text { Ditadi, 1982b; Dworschak \& Rodrigues, } 1997 \text { (como Pha- } \\
\text { coides pectinatus); Migotto et al. 1993; Belúcio, 1995; Arruda, } \\
\text { 2000; Arruda \& Amaral, 2003; Arruda et al. 2003; Denadai } \\
\text { et al. } 2005\end{array}$ & - \\
\hline Lunarca ovalis (Bruguière, 1789) & $\begin{array}{l}\text { Belúcio, 1995; Arruda, 2000; Arruda \& Amaral, 2003; Arruda } \\
\text { et al. } 2003 \text { (como Anadara ovalis); Migotto et al. } 1993\end{array}$ & - \\
\hline Lyonsia (Entodesma) beana (d'Orbigny, 1842) & $\begin{array}{l}\text { Duarte, 1980b; Morgado, 1985; Duarte \& Nalesso, 1996; } \\
\text { Morgado \& Tanaka, } 2001\end{array}$ & - \\
\hline Lyrodus floridanus (Bartsch, 1922) & Migotto et al. 1993 & - \\
\hline Lyrodus singaporeana (Roch, 1935) & Migotto et al. 1993 & - \\
\hline Macoma brevifrons (Say, 1834) & Ditadi, 1982b & - \\
\hline Macoma constricta (Bruguière, 1792) & $\begin{array}{l}\text { Ditadi, 1982b; Migotto et al. 1993; Belúcio, 1995; Arruda, } \\
\text { 2000; Arruda \& Amaral, 2003; Arruda et al. 2003; Denadai } \\
\text { et al. 2005; Padovanni, } 2009\end{array}$ & - \\
\hline Macoma uruguayensis (E.A. Smith, 1885) & $\begin{array}{l}\text { Migotto et al. 1993; Belúcio, 1995; Arruda, 2000; Arruda \& } \\
\text { Amaral, 2003; Arruda et al. 2003; Denadai et al. } 2005\end{array}$ & - \\
\hline Mactra fragilis Gmelin, 1791 & Ditadi, $1982 b$ & - \\
\hline Mactrellona alata (Spenger, 1802) & Padovanni, 2009 & - \\
\hline Martesia cuneiformis (Say, 1822) & Morgado, 1985; Morgado \& Tanaka, 2001 & - \\
\hline Martesia striata (Linnaeus, 1758) & Migotto et al. 1993 & - \\
\hline Modiolus americanus (Leach, 1815) & Nalesso et al. 1995 & - \\
\hline Modiolus carvalhoi Klappenbach, 1966 & $\begin{array}{l}\text { Duarte, 1980b; Morgado, 1980b, 1985; Migotto et al. 1993; } \\
\text { Nalesso et al. 1995; Duarte \& Nalesso, 1996; Morgado \& } \\
\text { Tanaka, 2001; Milanelli, } 2003\end{array}$ & - \\
\hline Mulinia cleryana (d'Orbigny, 1846) & Arruda, 2000; Arruda \& Amaral, 2003 & - \\
\hline Musculus lateralis (Say, 1822) & $\begin{array}{l}\text { Duarte, 1980b; Migotto et al. 1993; Nalesso et al. 1995; Duarte } \\
\text { \& Nalesso, } 1996\end{array}$ & - \\
\hline Nausitora fusticula (Jeffreys, 1860) & Migotto et al. 1993 & - \\
\hline Neoteredo reynei (Bartsch, 1920) & Migotto et al. 1993 & - \\
\hline Nucula semiornata d'Orbigny, 1846 & Belúcio \& Morgado, 1991; Belúcio, 1995 & - \\
\hline Ostrea equestris (Say, 1834) & Migotto et al. 1993 & - \\
\hline
\end{tabular}

HIBSP - Herbário Científico Maria Eneyda P. K. Fidalgo do Instituto de Botânica de São Paulo

MNRJ - Museu Nacional do Rio de Janeiro

MZUSP - Museu de Zoologia da Universidade de São Paulo

SPFUSP - Coleção de Algas do Departamento de Botânica da Universidade de São Paulo

USNM - National Museum of Natural History, Smithsonian Institution, USA 
Apêndice 1. Continuação...

\begin{tabular}{|c|c|c|}
\hline Táxon superior Espécie & Citação bibliográfica e/ou Coleção Científica & $\begin{array}{l}\text { Localidade- } \\
\text { tipo Araçá }\end{array}$ \\
\hline Periploma ovata d'Orbigny, 1846 & $\begin{array}{l}\text { Belúcio, 1995; Arruda, 2000; Arruda \& Amaral, 2003; Denadai } \\
\text { et al. } 2005\end{array}$ & - \\
\hline Perna perna (Linnaeus, 1758) & Migotto et al. 1993; Nalesso et al. 1995 & - \\
\hline Petricola stelae (Narchi, 1975) & Migotto et al. 1993 & - \\
\hline Pinctada radiata (Leach, 1814) & $\begin{array}{l}\text { Migotto et al. 1993; Nalesso et al. } 1995 \text { (como Pinctada } \\
\text { imbricata) }\end{array}$ & - \\
\hline Pinna carnea Gmelin, 1791 & Ditadi, $1982 b$ & - \\
\hline Pitar circinatus (Born, 1778) & Migotto et al. 1993 & - \\
\hline Protothaca pectorina (Lamarck, 1818) & Migotto et al. 1993; Belúcio, 1995; Padovanni, 2009 & - \\
\hline Rupellaria typica (Jonas, 1844) & Morgado, 1980b, 1985; Morgado \& Tanaka, 2001 & - \\
\hline Semele nuculoides (Conrad, 1841) & Migotto et al. 1993 & - \\
\hline Semele proficua (Pulteney, 1799) & $\begin{array}{l}\text { Migotto et al. 1993; Arruda, 2000; Amaral et al. 2003; Arruda } \\
\text { \& Amaral, 2003; Arruda et al. 2003; Denadai et al. } 2005\end{array}$ & - \\
\hline Semele purpurascens (Gmelin, 1791) & Denadai et al. 2005; MZUSP & - \\
\hline Sphenia antillensis Dall \& Simpson, 1901 & $\begin{array}{l}\text { Migotto et al. 1993; Belúcio, 1992, 1995; Belúcio \& Morgado, } \\
\text { 1994, } 1995\end{array}$ & - \\
\hline Strigilla carnaria (Linnaeus, 1758) & $\begin{array}{l}\text { Migotto et al. 1993; Belúcio \& Morgado, 1991; Belúcio, } \\
\text { 1995; Arruda \& Amaral, 2003; Arruda et al. 2003; Denadai } \\
\text { et al. } 2005\end{array}$ & - \\
\hline Strigilla pisiformis (Linnaeus, 1758) & Belúcio, 1995; Denadai et al. 2005 & - \\
\hline Strigilla producta Tryon, 1870 & Belúcio, 1995 & - \\
\hline Tagelus divisus (Spengler, 1794) & $\begin{array}{l}\text { Migotto et al. 1993; Belúcio, 1995; Arruda, 2000; Arruda } \\
\text { \& Amaral, 2003; Arruda et al. 2003; Denadai et al. 2005; } \\
\text { Padovanni, } 2009\end{array}$ & - \\
\hline Tagelus plebeius (Lightfoot, 1786) & $\begin{array}{l}\text { Migotto et al. 1993; Belúcio, 1995; Dworschak \& Rodrigues, } \\
\text { 1997; Arruda, 2000; Amaral et al. 2003; Arruda \& Amaral, } \\
\text { 2003; Arruda et al. 2003; Denadai et al. } 2005\end{array}$ & - \\
\hline Tagelus sp. & Belúcio, 1995 & - \\
\hline Tellina alternata Say, 1822 & Ditadi, $1982 b$ & - \\
\hline Tellina brasiliana Spengler, 1798 & Belúcio, 1995 & - \\
\hline Tellina exerythra Boss, 1964 & Belúcio, 1995; Padovanni, 2009 & - \\
\hline Tellina iheringi Dall, 1900 & Belúcio, 1995 & - \\
\hline Tellina lineata Turton, 1819 & $\begin{array}{l}\text { Belúcio, 1995; Arruda, 2000; Arruda \& Amaral, 2003; Arruda } \\
\text { et al. 2003; Denadai et al. 2005; Padovanni, } 2009\end{array}$ & - \\
\hline Tellina punicea Born, 1778 & Migotto et al. 1993 & - \\
\hline Tellina versicolor De Kay, 1843 & $\begin{array}{l}\text { Migotto et al. 1993; Belúcio, 1995; Arruda, 2000; Arruda \& } \\
\text { Amaral, 2003; Arruda et al. 2003; Denadai et al. } 2005\end{array}$ & - \\
\hline Teredo bartschi Clapp, 1923 & Migotto et al. 1993 & - \\
\hline Teredo navalis Linnaeus, 1758 & Migotto et al. 1993 & - \\
\hline Teredo triangularis Edmondson, 1942 & Migotto et al. 1993 & - \\
\hline Tivela mactroides (Born, 1778) & Migotto et al. 1993; Belúcio, 1995 & - \\
\hline Trachycardium muricatum (Linnaeus, 1758) & Ditadi, 1982b & - \\
\hline ANNELIDA, POLYCHAETA & & - \\
\hline Aonides sp. & Lopes, 1993 & - \\
\hline Amblyosyllis sp. & Fukuda (em andamento) & - \\
\hline Ammotrypane aulogaster Rathke, 1843 & Burdon-Jones \& Petersen, 1964; Ditadi, 1982b & - \\
\hline Amphiglena lindae Rouse \& Gambi, 1997 & Rossi, 2008 & - \\
\hline
\end{tabular}

HIBSP - Herbário Científico Maria Eneyda P. K. Fidalgo do Instituto de Botânica de São Paulo

MNRJ - Museu Nacional do Rio de Janeiro

MZUSP - Museu de Zoologia da Universidade de São Paulo

SPFUSP - Coleção de Algas do Departamento de Botânica da Universidade de São Paulo

USNM - National Museum of Natural History, Smithsonian Institution, USA 
Apêndice 1. Continuação...

\begin{tabular}{|c|c|c|}
\hline Táxon superior Espécie & Citação bibliográfica e/ou Coleção Científica & $\begin{array}{l}\text { Localidade- } \\
\text { tipo Araçá }\end{array}$ \\
\hline Amphinome sp. & Morgado \& Tanaka, 2001 & - \\
\hline Apoprionospio sp. & Lopes, 1993 & - \\
\hline Arabella aracaensis Steiner \& Amaral, 2009 & Steiner, 2000 (como Arabella sp.n.); Steiner \& Amaral, 2009 & - \\
\hline Arenicola sp. & Burdon-Jones \& Petersen, 1964; Rodrigues, 1971 & - \\
\hline Aricidea simplex Day, 1963 & Lopes, 1993 (como Aricidea (Acmira) simplex) & - \\
\hline Aricidea fragilis Webster, 1879 & Lopes, 1993; Amaral \& Morgado, 1994 & - \\
\hline Aricidea pseudoarticulata Hobson, 1972 & Lopes, 1993 & - \\
\hline Aricidea suecica Eliason, 1920 & Lopes, 1993 & - \\
\hline Armandia agilis (Andrews, 1891) & $\begin{array}{l}\text { Lopes, 1993; Amaral et al. 1994, 2003; Reis et al. 1996; Omena\& } \\
\text { Amaral, 1997; Reis \& Amaral, 1998; Padovanni, } 2009\end{array}$ & - \\
\hline Armandia maculata (Webster, 1884) & Morgado \& Tanaka, 2001 & - \\
\hline Bhawania sp. & $\begin{array}{l}\text { Morgado \& Amaral, 1981b; Morgado \& Tanaka, } 2001 \text { (como } \\
\text { Bhawania brunnea) }\end{array}$ & - \\
\hline Bhawania goodei Webster, 1884 & Ditadi, 1970a; Ditadi, 1982b; Anker et al. 2005 & - \\
\hline Boccardia redeki (Horst, 1920) & $\begin{array}{l}\text { Duarte, 1980b; Morgado, 1980b; Duarte \& Nalesso, 1996; } \\
\text { Morgado \& Tanaka, } 2001\end{array}$ & - \\
\hline Boccardia sp. & Lopes, 1993 & - \\
\hline Branchiomma patriota Nogueira, Rossi \& López, 2006 & $\begin{array}{l}\text { Duarte, 1980b; Lopes, 1993; Duarte \& Nalesso, 1996; Morgado } \\
\text { \& Tanaka, 2001 (como Branchiomma nigromaculata); Rossi \& } \\
\text { Nogueira, 2004; Nogueira et al. 2006; Rossi, } 2008\end{array}$ & - \\
\hline Branchiosyllis exilis (Gravier, 1900) & Fukuda (em andamento) & - \\
\hline Capitellidae sp. 1 & Duarte, 1980b; Duarte \& Nalesso, 1996 & - \\
\hline Capitellidae sp. 2 & Duarte, 1980b; Duarte \& Nalesso, 1996 & - \\
\hline Capitellidae sp. 3 & Duarte, 1980b; Duarte \& Nalesso, 1996 & - \\
\hline Capitellidae sp. & Lopes, 1993; Morgado \& Tanaka, 2001 & - \\
\hline Capitomastus minimus (Langerhans, 1880) & Lopes, 1993 & - \\
\hline Ceratocephale sp. & Lopes, 1993 & - \\
\hline Chaetopterus sp. & $\begin{array}{l}\text { Sawaya, \& Freitas, 1972; Ditadi, 1982b; Zinner \& Vani, 1986; } \\
\text { Vani, 1987; Dworschak \& Rodrigues, 1997; Anker et al. } 2005 \\
\text { (como Chaetopterus variopedatus) }\end{array}$ & - \\
\hline Chrysopetalum occidentale Johnson, 1897 & $\begin{array}{l}\text { Morgado, } 1980 \mathrm{~b} \text {; Morgado \& Amaral, 1981b; Morgado \& } \\
\text { Tanaka, } 2001 \text { (como Chaetopterus variopedatus) }\end{array}$ & - \\
\hline Cirriformia filigera (Delle Chiaje, 1825) & $\begin{array}{l}\text { Duarte, 1980b; Morgado, 1980b; Duarte \& Nalesso, 1996; } \\
\text { Morgado \& Tanaka, } 2001\end{array}$ & - \\
\hline Cirriformia tentaculata (Montagu, 1808) & Amaral \& Morgado, 1987; Lopes, 1993 & - \\
\hline Cirrophorus sp. & Lopes, 1993 & - \\
\hline Clymenella brasiliensis Mangum, 1966 & Lopes, 1993 & - \\
\hline Complexo Capitella capitata (Fabricius, 1780) & $\begin{array}{l}\text { Amaral \& Morgado, 1987, 1989, 1994; Morgado \& Amaral, } \\
\text { 1988; Amaral et al. 1989, 1990, 1994, 1998, 2003; Lopes, 1993; } \\
\text { Omena\& Amaral, 1997; Reis \& Amaral, 1998; Reis et al. 1996; } \\
\text { Padovanni, } 2009 \text { (como Capitella sp.) }\end{array}$ & - \\
\hline Cossura delta Reish, 1958 & Lopes, 1993 & - \\
\hline Demonax cf. microphthalmus Spencer, 1973 & Rossi, 2008 & - \\
\hline Diopatra aciculata Knox \& Cameron, 1971 & $\begin{array}{l}\text { Steiner, 2000, } 2005 \text { (como Diopatra neopolitana); Padovanni, } \\
2009\end{array}$ & - \\
\hline
\end{tabular}

HIBSP - Herbário Científico Maria Eneyda P. K. Fidalgo do Instituto de Botânica de São Paulo

MNRJ - Museu Nacional do Rio de Janeiro

MZUSP - Museu de Zoologia da Universidade de São Paulo

SPFUSP - Coleção de Algas do Departamento de Botânica da Universidade de São Paulo

USNM - National Museum of Natural History, Smithsonian Institution, USA 
Apêndice 1. Continuação...

\begin{tabular}{|c|c|c|}
\hline Táxon superior Espécie & Citação bibliográfica e/ou Coleção Científica & $\begin{array}{l}\text { Localidade- } \\
\text { tipo Araçá }\end{array}$ \\
\hline Diopatra cuprea (Bosc, 1802) & $\begin{array}{l}\text { Ditadi, 1982b; Lopes, 1993; Amaral \& Morgado, 1994; Amaral } \\
\text { et al. 1994; Dworschak \& Rodrigues, 1997; Steiner \& Amaral, } \\
\text { 1998; Steiner, 2000; Amaral et al. } 2008\end{array}$ & - \\
\hline Diopatra ornata Moore, 1911 & Lopes, 1993; Amaral \& Morgado, 1994 & - \\
\hline Diopatra splendidissima Kinberg, 1857 & Lopes, 1993 & - \\
\hline Dodecaceria concharum Örsted, 1843 & Morgado, 1980b; Morgado \& Tanaka, 2001 & - \\
\hline Dorvillea sociabilis (Webster, 1879) & $\begin{array}{l}\text { Morgado, 1980b; Morgado \& Amaral, 1981a; Morgado \& } \\
\text { Tanaka, } 2001\end{array}$ & - \\
\hline Eteone heteropoda Hartman, 1951 & Lopes, 1993 & - \\
\hline Eulalia viridis (Linnaeus, 1767) & $\begin{array}{l}\text { Morgado, 1980b; Morgado \& Amaral, 1984a,b; Morgado \& } \\
\text { Tanaka, } 2001\end{array}$ & - \\
\hline Eumida sanguinea (Örsted, 1843) & $\begin{array}{l}\text { Duarte, 1980b; Morgado, 1980b; Morgado \& Amaral, 1984a,b; } \\
\text { Duarte \& Nalesso, 1996; Morgado \& Tanaka, } 2001\end{array}$ & - \\
\hline Eunice binominata Quatrefages, 1866 & Morgado \& Tanaka, 2001 & - \\
\hline Eunice cariboea (Grube, 1856) & $\begin{array}{l}\text { Duarte, 1980b; Morgado, 1980b; Morgado \& Amaral, 1981a; } \\
\text { Duarte \& Nalesso, 1996; Morgado \& Tanaka, } 2001\end{array}$ & - \\
\hline Eunice filamentosa Grube, 1856 & Morgado \& Amaral, 1981a & - \\
\hline Eunice rubra Grube, 1856 & $\begin{array}{l}\text { Duarte, 1980b; Morgado \& Amaral, 1981a; Duarte \& Nalesso, } \\
\text { 1996; Morgado \& Tanaka, } 2001\end{array}$ & - \\
\hline Eunice sebastiani Nonato, 1965 & $\begin{array}{l}\text { Burdon-Jones \& Petersen, } 1964 \text { (como Eunice rousseaui); } \\
\text { Nonato, 1965; Faria, 1974; Fauchald, 1992; Carrera-Parra et al. } \\
\text { 2008; Amaral, et al. } 2008\end{array}$ & - \\
\hline Eunice $\mathrm{cf}$. tenuis (Treadwell, 1921) & $\begin{array}{l}\text { Morgado, 1980b; Morgado \& Amaral, 1981a; Morgado \& } \\
\text { Tanaka, } 2001\end{array}$ & - \\
\hline $\begin{array}{l}\text { Exogone (Exogone) breviantennata Hartmann-Schröder, } \\
1959\end{array}$ & $\begin{array}{l}\text { Duarte, 1980b; Morgado \& Amaral, 1985; Lopes, 1993; Duarte } \\
\text { \& Nalesso, 1996; Morgado \& Tanaka, } 2001 \text { (como Exogone } \\
\text { occidentallis) }\end{array}$ & - \\
\hline Exogone (Exogone) sp. & Fukuda (em andamento) & - \\
\hline Fabriciola sp. n. & Rossi, 2008 & - \\
\hline Glycera americana Leidy, 1855 & Lopes, 1993 & - \\
\hline Glycera dibranchiata Ehlers, 1868 & Steiner, 2000; Rizzo et al. 2007 & - \\
\hline Glycera oxycephala Ehlers, 1887 & Lopes, 1993 & - \\
\hline Glycinde multidens Müller, 1858 & $\begin{array}{l}\text { Morgado \& Amaral, 1988; Lopes, 1993; Amaral \& Morgado, } \\
\text { 1994; Amaral et al. 1994, 2003; Reis \& Amaral, 1998; Steiner } \\
\text { \& Amaral, 1998; Steiner, } 2000 \text { (como Glycinde sp.); Padovanni, } \\
2009\end{array}$ & - \\
\hline Goniada littorea Hartman, 1950 & Lopes, 1993; Amaral \& Morgado, 1994; Steiner, 2000 & - \\
\hline Goniada maculata Hartman, 1950 & Lopes, 1993 & - \\
\hline Goniada sp. & Lopes, 1993 & - \\
\hline Goniadides carolinae Day, 1973 & Steiner, 2000 & - \\
\hline Gymnonereis cf. crosslandi (Monro, 1933) & Steiner, 2000 & - \\
\hline Gyptis brevipalpa (Hartmann-Schröder, 1959) & Lopes, 1993 & - \\
\hline Halosydna glabra Hartman, 1939 & Morgado \& Amaral, 1981c; Morgado \& Tanaka, 2001 & - \\
\hline Haploscoloplos fragilis (Verrill, 1873) & Lopes, 1993 & - \\
\hline Haploscoloplos sp. & Lopes, 1993; Amaral \& Morgado, 1994 & - \\
\hline Haplosyllis spongicola (Grube, 1855) & $\begin{array}{l}\text { Duarte, 1980b; Morgado \& Amaral, 1985; Duarte \& Nalesso, } \\
\text { 1996; Morgado \& Tanaka, } 2001\end{array}$ & - \\
\hline
\end{tabular}

HIBSP - Herbário Científico Maria Eneyda P. K. Fidalgo do Instituto de Botânica de São Paulo

MNRJ - Museu Nacional do Rio de Janeiro

MZUSP - Museu de Zoologia da Universidade de São Paulo

SPFUSP - Coleção de Algas do Departamento de Botânica da Universidade de São Paulo

USNM - National Museum of Natural History, Smithsonian Institution, USA 
Apêndice 1. Continuação...

\begin{tabular}{|c|c|c|}
\hline Táxon superior Espécie & Citação bibliográfica e/ou Coleção Científica & $\begin{array}{l}\text { Localidade- } \\
\text { tipo Araçá }\end{array}$ \\
\hline Haplosyllis sp. & Fukuda (em andamento) & - \\
\hline Harmothoe aculeata Andrews, 1891 & Lopes, 1993 & - \\
\hline Harmothoe imbricata (Linnaeus, 1767) & Ditadi, 1970a; Ditadi, 1982b; Anker et al. 2005 & - \\
\hline Harmothoe macginitiei Pettibone, 1955 & $\begin{array}{l}\text { Duarte, 1980b; Duarte \& Nalesso, 1996; Morgado \& Tanaka, } \\
2001\end{array}$ & - \\
\hline Harmothoe sp. & Morgado \& Tanaka, 2001 & - \\
\hline Hemipodia californiensis (Hartman 1938) & $\begin{array}{l}\text { Steiner \& Amaral, 1998; Lopes, } 1993 \text { (como Hemipodus } \\
\text { olivieri); Steiner, } 2000 \text { (como Hemipodus rotundus); Rizzo } \\
\text { et al. } 2007\end{array}$ & - \\
\hline Hemipodia simplex (Grube 1857) & $\begin{array}{l}\text { Lopes, } 1993 \text { Steiner \& Amaral, } 1998 \text { (como Hemipodus } \\
\text { rotundus); Steiner, } \\
2000 \text { (como Hemipodus simplex) }\end{array}$ & - \\
\hline Heteromastus filiformis (Claparède, 1864) & $\begin{array}{l}\text { Amaral \& Morgado, 1987, 1989, 1994; Morgado \& Amaral, } \\
\text { 1988; Amaral et al. 1989, 1990, 1994; 1998, 2003; Lopes, } \\
\text { 1993; Reis et al. 1996; Omena\& Amaral, 1997; Reis \& Amaral, } \\
\text { 1997, 1998; Morgado \& Amaral, 1998; Padovanni, } 2009 \text { (como } \\
\text { Heteromastus sp.) }\end{array}$ & - \\
\hline Hydroides brachyacanthus Rioja, 1941 & Morgado \& Tanaka, 2001 & - \\
\hline Hydroides diramphus Mörch, 1863 & Morgado \& Tanaka, 2001 & - \\
\hline Hypsicomus elegans (Webster, 1884) & Morgado, 1980b; Morgado \& Tanaka, 2001 & - \\
\hline Isolda pulchella F. Müller, 1858 & $\begin{array}{l}\text { Amaral \& Morgado, 1987, 1994; Lopes, 1993; Amaral et al. } \\
\text { 1994, 1998, 2003; Padovanni, } 2009\end{array}$ & - \\
\hline Kirkia heterobranchiata (gen. sp. nov.) & Nogueira et al. 2004a; Rossi, 2008 & - \\
\hline Labrorostratus prolificus Amaral, 1977 & Steiner, 2000; Steiner \& Amaral, 2009 & - \\
\hline Laeonereis culveri (Webster, 1879) & $\begin{array}{l}\text { Amaral \& Morgado, 1987, 1989, 1994; Amaral et al. 1989, } \\
\text { 1994, 1998, 2003; Lopes, 1993; Amaral \& Morgado, 1994; } \\
\text { Omena \& Amaral, 1997; Reis \& Amaral, 1997, 1998; Steiner } \\
\text { \& Amaral, 1998; Steiner, } 2000 \text { (como Laeonereis acuta) }\end{array}$ & - \\
\hline Langerhansia cornuta (Rathke, 1843) & Lopes, 1993 & - \\
\hline Laonice sp. & Lopes, 1993 & - \\
\hline Lepidonotus caeruleus Kinberg, 1855 & $\begin{array}{l}\text { Morgado \& Amaral, 1981c; Morgado, 1980b; Morgado \& } \\
\text { Tanaka, } 2001\end{array}$ & - \\
\hline Loimia medusa (Savigny, 1818) & Lopes, 1993; Morgado \& Tanaka, 2001 & - \\
\hline Loimia sp. & Ditadi, $1982 b$ & - \\
\hline Lumbrineris albifrons Crossland, 1924 & $\begin{array}{l}\text { Duarte, 1980b; Morgado, 1980b; Morgado \& Amaral, 1981a; } \\
\text { Duarte \& Nalesso, 1996; Morgado \& Tanaka, } 2001\end{array}$ & - \\
\hline Lumbrineris tetraura (Schmarda, 1861) & Lopes, 1993; Amaral \& Morgado, 1994; Steiner, 2000 & - \\
\hline Lysibranchia sp. & Lopes, 1993 & - \\
\hline Lysidice ninetta A. \& M.Edwards, 1833 & $\begin{array}{l}\text { Morgado \& Amaral, 1981a; Lopes, 1993; Duarte \& Nalesso, } \\
\text { 1996; Steiner \& Amaral, 1998; Morgado \& Tanaka, } 2001\end{array}$ & - \\
\hline Magelona nonatoi Bolivar \& Lana, 1986 & Lopes, 1993 & - \\
\hline Magelona papillicornis F. Müller, 1858 & Lopes, 1993; Amaral \& Morgado, 1994 & - \\
\hline Magelona posterelongata Bolivar \& Lana, 1986 & Lopes, 1993 & - \\
\hline Magelona riojai Jones, 1963 & Lopes, 1993 & - \\
\hline Magelona variolamellata Bolivar \& Lana, 1986 & Lopes, 1993 & - \\
\hline Magelona sp. & Lopes, 1993; Omena \& Amaral, 1997 & - \\
\hline Marphysa angelensis Fauchald, 1970 & $\begin{array}{l}\text { Duarte, 1980b; Morgado \& Amaral, 1981a; Duarte \& Nalesso, } \\
\text { 1996; Morgado \& Tanaka, } 2001\end{array}$ & - \\
\hline
\end{tabular}

HIBSP - Herbário Científico Maria Eneyda P. K. Fidalgo do Instituto de Botânica de São Paulo

MNRJ - Museu Nacional do Rio de Janeiro

MZUSP - Museu de Zoologia da Universidade de São Paulo

SPFUSP - Coleção de Algas do Departamento de Botânica da Universidade de São Paulo

USNM - National Museum of Natural History, Smithsonian Institution, USA 
Apêndice 1. Continuação...

\begin{tabular}{|c|c|c|}
\hline Táxon superior Espécie & Citação bibliográfica e/ou Coleção Científica & $\begin{array}{l}\text { Localidade- } \\
\text { tipo Araçá }\end{array}$ \\
\hline Marphysa formosa Steiner \& Amaral, 2000 & Amaral et al. 2003 & - \\
\hline Marphysa sanguinea (Montagu, 1815) & $\begin{array}{l}\text { Morgado \& Amaral, 1981a; Lopes, 1993; Morgado \& Tanaka, } \\
2001\end{array}$ & - \\
\hline Marphysa sebastiana Steiner \& Amaral, 2000 & Steiner, 2000; Steiner \& Amaral, 2000; Padovanni, 2009 & - \\
\hline Marphysa sp. & Lopes, 1993; Reis \& Amaral, 1998 & - \\
\hline Mediomastus californiensis Hartman, 1944 & Lopes, 1993 & - \\
\hline Megalomma sp. & Lopes, 1993 & - \\
\hline Mesochaetopterus xejubus Petersen \& Fanta, 1969 & Ditadi, 1982b & - \\
\hline Mesochaetopterus xerecus Petersen \& Fanta, 1969 & Ditadi, $1982 b$ & - \\
\hline Naineris setosa (Verrill, 1900) & $\begin{array}{l}\text { Ditadi, 1970a; Ditadi, 1982b; Lopes, 1993; Amaral et al. 2003; } \\
\text { Anker et al. } 2005\end{array}$ & - \\
\hline Naineris sp. & Lopes, 1993 & - \\
\hline Neanthes bruaca Lana \& Sovierzovsky, 1987 & Steiner \& Amaral, 1998, Steiner, 2000 & - \\
\hline Neanthes ceciliae Steiner \& Santos, 2004 & $\begin{array}{l}\text { Steiner, } 2000 \text { (como Neanthes sp.); Steiner \& Santos, 2004; } \\
\text { Avellar et al. } 2006\end{array}$ & - \\
\hline Neanthes succinea (Frey \& Leuckart, 1847) & $\begin{array}{l}\text { Duarte, 1980b; Amaral \& Morgado, 1994; Duarte \& Nalesso, } \\
\text { 1996; Morgado \& Tanaka, 2001; Avellar et al. } 2006\end{array}$ & - \\
\hline Neanthes sp. & Lopes, 1993 & - \\
\hline Nematonereis hebes Verrill, 1900 & $\begin{array}{l}\text { Morgado, 1980b; Morgado \& Amaral, 1981a; Steiner \& Am- } \\
\text { ral, 1998; Steiner, 2000; Morgado \& Tanaka, 2001; Amaral } \\
\text { et al. } 2003\end{array}$ & - \\
\hline Nematonereis unicornis Schmarda, 1861 & Ditadi, 1970a; Ditadi, 1982b; Anker et al. 2005 & - \\
\hline Nephtys sp. & Lopes, 1993 & - \\
\hline Nereis pseudonereis Hartman, 1940 & Lopes, 1993 & - \\
\hline Nereis riisei Grube, 1857 & $\begin{array}{l}\text { Duarte, 1980b; Morgado, 1980b; Duarte \& Nalesso, 1996; } \\
\text { Morgado \& Tanaka, 2001; Avellar et al. } 2006\end{array}$ & - \\
\hline Nicolea uspiana (Nogueira, 2003) & $\begin{array}{l}\text { Nogueira, } 2003 \text { (como Paraeupolymnia uspiana); Alves, } \\
2008\end{array}$ & - \\
\hline Nicon sp. 1 & Avellar et al. 2006 & - \\
\hline Nicon sp. 2 & Avellar et al. 2006 & - \\
\hline Ninoe brasiliensis Kinberg, 1865 & Lopes, 1993 & - \\
\hline Notaulax sp.n. 1 & Nogueira et al. 2004a; Rossi, 2008 & - \\
\hline Notomastus hemipodus Hartman, 1945 & Lopes, 1993 & - \\
\hline Notomastus lobatus Hartman, 1947 & Lopes, 1993 & - \\
\hline Notomastus sp. & Morgado, 1980b; Lopes, 1993; Morgado \& Tanaka, 2001 & - \\
\hline Odontosyllis guillermoi Fukuda \& Nogueira, 2006 & Fukuda \& Nogueira, 2004 (como Odontosyllis sp.) & - \\
\hline Oenone diphyllidia Schmarda, 1861 & Morgado \& Amaral, 1981a; Morgado \& Tanaka, 2001 & - \\
\hline Onuphis vexillaria Moore, 1911 & Lopes, 1993 & - \\
\hline Ophiodromus pallidus (Claparède, 1864) & Ditadi, 1982b; Anker et al. 2005 & - \\
\hline Ophiodromus pugettensis (Johnson, 1901) & $\begin{array}{l}\text { Duarte, 1980b; Morgado, 1980b; Morgado \& Amaral, 1984a,b; } \\
\text { Lopes, 1993; Duarte \& Nalesso, 1996; Morgado \& Tanaka, } \\
2001\end{array}$ & - \\
\hline Opisthosyllis brunnea Langerhans, 1879 & Fukuda (em andamento) & - \\
\hline Opisthosyllis corallicola Hartmann-Schröder, 1965 & Morgado \& Amaral, 1985; Morgado \& Tanaka, 2001 & - \\
\hline Owenia fusiformis delle Chiaje, 1841 & Lopes, 1993; Amaral \& Morgado, 1994; Amaral et al. 1994 & - \\
\hline Palola esbelta Morgado \& Amaral, 1981 & Morgado \& Amaral, 1981a; Morgado \& Tanaka, 2001 & - \\
\hline
\end{tabular}

HIBSP - Herbário Científico Maria Eneyda P. K. Fidalgo do Instituto de Botânica de São Paulo

MNRJ - Museu Nacional do Rio de Janeiro

MZUSP - Museu de Zoologia da Universidade de São Paulo

SPFUSP - Coleção de Algas do Departamento de Botânica da Universidade de São Paulo

USNM - National Museum of Natural History, Smithsonian Institution, USA 
Apêndice 1. Continuação...

\begin{tabular}{|c|c|c|}
\hline Táxon superior Espécie & Citação bibliográfica e/ou Coleção Científica & $\begin{array}{l}\text { Localidade- } \\
\text { tipo Araçá }\end{array}$ \\
\hline Paradoneis cf. lyra (Southern, 1914) & $\begin{array}{l}\text { Duarte, 1980b; Duarte \& Nalesso, 1996; Morgado \& Tanaka, } \\
2001\end{array}$ & - \\
\hline Parandalia americana (Hartman, 1947) & $\begin{array}{l}\text { Lopes, 1993; Reis \& Amaral, } 1998 \text { (como Loandalia } \\
\text { americana); Amaral et al. } 2003\end{array}$ & - \\
\hline Paraprionospio pinnata (Ehlers, 1901) & Lopes, 1993 & - \\
\hline Pectinaria sp. & Lopes, 1993 & - \\
\hline Perinereis anderssoni Kinberg, 1866 & Steiner \& Amaral, 1998; Avellar et al. 2006 & - \\
\hline Perinereis cultrifera (Grube, 1840) & Lopes, 1993; Steiner, 2000 & - \\
\hline Perinereis ponteni Kinberg, 1866 & Avellar et al. 2006 & - \\
\hline Phisidia rubra Nogueira \& Alves, 2006 & Nogueira \& Alves, 2006; Alves, 2008 & - \\
\hline Phyllochaetopterus socialis Claparède, 1870 & Nalesso et al. 1995 & - \\
\hline Phyllodoce sp. & Lopes, 1993 & - \\
\hline Phylo felix Kinberg, 1866 & Lopes, 1993 & - \\
\hline Pista herpini Fauvel, 1928 & $\begin{array}{l}\text { Duarte, 1980b; Duarte \& Nalesso, 1996; Morgado \& Tanaka, } \\
2001\end{array}$ & - \\
\hline Platynereis dumerilii (Audouin and Milne-Edwards, 1833) & Steiner \& Amaral, 1998; Steiner, 2000; Avellar et al. 2006 & - \\
\hline Platynereis sp. & Lopes, 1993 & - \\
\hline Podarke pallida (Claparède, 1864) & Ditadi, 1970a & - \\
\hline Podarke sp. & Lopes, 1993 & - \\
\hline Poecilochaetus australis Nonato, 1963 & Lopes, 1993 & - \\
\hline Polycirrus hamiltoni Benham, 1921 & $\begin{array}{l}\text { Duarte, 1980b; Morgado, 1980b; Duarte \& Nalesso, 1996; } \\
\text { Morgado \& Tanaka, } 2001\end{array}$ & - \\
\hline Polycirrus sp. & Alves, 2008 & - \\
\hline Polydora ligni Webster, 1879 & Lopes, 1993 & - \\
\hline Polydora websteri Hartman, 1943 & Morgado, 1980b; Morgado \& Tanaka, 2001 & - \\
\hline Polydora sp. & Nogueira et al. 2004a,b & - \\
\hline Pomatocerus minutus Rioja, 1941 & Morgado, 1980b; Morgado \& Tanaka, 2001 & - \\
\hline Prionospio dayi (Foster, 1969) & Lopes, 1993 & - \\
\hline Prionospio heterobranchia Moore, 1907 & Lopes, 1993 & - \\
\hline Prionospio steenstrupi Malmgren, 1867 & Lopes, 1993 & - \\
\hline Proceraea okadai (Imajima, 1966) & Fukuda (em andamento) & - \\
\hline Proceraea sp. & Morgado \& Tanaka, 2001 (como Autolytus sp.) & - \\
\hline $\begin{array}{l}\text { Pseudobranchiomma paraemersoni Nogueira, Rossi \& } \\
\text { López, } 2006\end{array}$ & $\begin{array}{l}\text { Duarte, 1980b; Duarte \& Nalesso, } 1996 \text { (como Pseudobranchiomma } \\
\text { emersoni); Nogueira et al. 2006; Rossi, } 2008\end{array}$ & - \\
\hline $\begin{array}{l}\text { Pseudobranchiomma paulista Nogueira, Rossi \& López, } \\
2006\end{array}$ & Nogueira et al. 2006; Rossi, 2008 & - \\
\hline Pseudonereis gallapaguensis Kinberg, 1865 & Avellar et al. 2006 & - \\
\hline Pseudonereis palpata (Treadwell, 1923) & Avellar et al. 2006 & - \\
\hline Pseudonereis sp. nov. & Duarte, $1980 b$ & - \\
\hline Pseudonereis sp. & $\begin{array}{l}\text { Morgado, 1980b; Duarte \& Nalesso, 1996; Morgado \& Tanaka, } \\
2001\end{array}$ & - \\
\hline Pseudopotamilla reniformis (Müller, 1771) & Morgado \& Tanaka, 2001 (como Potamilla reniformis) & - \\
\hline Pseudopotamilla sp. n. & Rossi, 2008 & - \\
\hline $\begin{array}{l}\text { Pseudostreblosoma brevitentaculatum Nogueira \& Alves, } \\
2006\end{array}$ & Nogueira \& Alves, 2006 & - \\
\hline Sabellaria floridensis Hartman, 1944 & $\begin{array}{l}\text { Duarte, 1980b; Duarte \& Nalesso, 1996; Morgado \& Tanaka, } \\
2001\end{array}$ & - \\
\hline
\end{tabular}

HIBSP - Herbário Científico Maria Eneyda P. K. Fidalgo do Instituto de Botânica de São Paulo

MNRJ - Museu Nacional do Rio de Janeiro

MZUSP - Museu de Zoologia da Universidade de São Paulo

SPFUSP - Coleção de Algas do Departamento de Botânica da Universidade de São Paulo

USNM - National Museum of Natural History, Smithsonian Institution, USA 
Apêndice 1. Continuação...

\begin{tabular}{|c|c|c|}
\hline Táxon superior Espécie & Citação bibliográfica e/ou Coleção Científica & $\begin{array}{l}\text { Localidade- } \\
\text { tipo Araçá }\end{array}$ \\
\hline Salvatoria nitidula (Verrill, 1900) & Fukuda (em andamento) & - \\
\hline Scalisetosus gracilis Morgado \& Amaral, 1981 & $\begin{array}{l}\text { Morgado \& Amaral, 1981b; Duarte \& Nalesso, 1996; Morgado } \\
\text { \& Tanaka, } 2001\end{array}$ & - \\
\hline Scalisetosus pellucidus (Ehlers, 1864) & Duarte, 1980b; Morgado, 1980b & - \\
\hline Scolelepis chilensis (Hartmann-Schröder, 1962) & $\begin{array}{l}\text { Lopes, 1993; Amaral \& Morgado, 1994; Amaral et al. 1994, } \\
\text { 1998, } 2003 \text { (como Scolelepis squamata) }\end{array}$ & - \\
\hline Scoloplos (Leodamas) gracilis Pillai, 1961 & Lopes, 1993 & - \\
\hline Scoloplos (Leodamas) johnstonei (Day, 1934) & Lopes, 1993 & - \\
\hline Scoloplos (Leodamas) ohlini (Ehlers, 1901) & Lopes, 1993 & - \\
\hline Scoloplos (Leodamas) sp.n. & $\begin{array}{l}\text { Amaral \& Morgado, 1987, 1994; Morgado \& Amaral, 1988; } \\
\text { Lopes, 1993; Amaral et al. 1994, 1998, 2003; Reis et al. 1996; } \\
\text { Omena\& Amaral, 1997; Padovanni, 2009 }\end{array}$ & - \\
\hline Scoloplos texana Maciolek \& Holland, 1978 & Lopes, 1993 & - \\
\hline Sigambra grubei Müller, 1858 & Lopes, 1993; Amaral et al. 1994 & - \\
\hline Spiophanes missionensis Hartman, 1941 & Lopes, 1993; & - \\
\hline Stauronereis rudolphi (Delle Chiaje, 1828) & Morgado \& Amaral, 1981a & - \\
\hline Sternaspis capillata Nonato, 1966 & Lopes, 1993 & - \\
\hline Sthenelais limicola (Ehlers, 1864) & Lopes, 1993 & - \\
\hline Streblosoma bairdi (Malmgren, 1866) & Morgado \& Tanaka, 2001 & - \\
\hline Syllidae sp. E & Morgado \& Tanaka, 2001 & - \\
\hline Syllis corallicola Verrill, 1900 & Fukuda (em andamento) & - \\
\hline Syllis gracilis Grube, 1840 & $\begin{array}{l}\text { Morgado, 1980b; Morgado \& Amaral, 1985; Morgado \& } \\
\text { Tanaka, } 2001\end{array}$ & - \\
\hline Syllis magellanica Augener, 1918 & Fukuda (em andamento) & - \\
\hline Syllis prolifera Krohn, 1852 & Fukuda (em andamento) & - \\
\hline Syllis pseudoarmillaris Nogueira \& San Martín, 2002 & Fukuda (em andamento) & - \\
\hline Syllis rosea Langerhans, 1879 & Fukuda (em andamento) & - \\
\hline Syllis westheidei San Martín, 1984 & Fukuda (em andamento) & - \\
\hline Syllis sp.1 & Fukuda (em andamento) & - \\
\hline Terebella cf. pterochaeta Schmarda, 1861 & Morgado, 1980b; Morgado \& Tanaka, 2001 & - \\
\hline Terebella sp. 1 & Alves, 2008 & - \\
\hline Terebellides anguicomus Müller, 1858 & Lopes, 1993; Padovanni, 2009 & - \\
\hline Tharyx filibranchia Day, 1961 & Lopes, 1993 & - \\
\hline Tharyx sp. & Lopes, 1993 & - \\
\hline Thelepsavus sp. & Ditadi, $1982 b$ & - \\
\hline Trypanosyllis taeniaformis (Haswell, 1886) & $\begin{array}{l}\text { Duarte, 1980b; Morgado, 1980b; Morgado \& Amaral, 1985; } \\
\text { Duarte \& Nalesso, 1996; Morgado \& Tanaka, } 2001\end{array}$ & - \\
\hline Trypanosyllis zebra (Grube, 1840) & Nogueira \& Fukuda, 2007; Nogueira \& Fukuda, 2008 & - \\
\hline Typosyllis hyalina (Grube, 1863) & Duarte, 1980b; Morgado, 1980b & - \\
\hline Typosyllis maculata Imajima, 1966 & $\begin{array}{l}\text { Duarte, 1980b; Morgado \& Amaral, 1985; Duarte \& Nalesso, } \\
\text { 1996; Morgado \& Tanaka, } 2001\end{array}$ & - \\
\hline Typosyllis variegata (Grube, 1860 & $\begin{array}{l}\text { Morgado \& Amaral, 1985; Duarte \& Nalesso, 1996; Morgado } \\
\text { \& Tanaka, } 2001\end{array}$ & - \\
\hline ECHIURA & & - \\
\hline Arhynchite paulensis Amor, A., 1971 & Amor, 1971 & - \\
\hline
\end{tabular}

HIBSP - Herbário Científico Maria Eneyda P. K. Fidalgo do Instituto de Botânica de São Paulo

MNRJ - Museu Nacional do Rio de Janeiro

MZUSP - Museu de Zoologia da Universidade de São Paulo

SPFUSP - Coleção de Algas do Departamento de Botânica da Universidade de São Paulo

USNM - National Museum of Natural History, Smithsonian Institution, USA 
Apêndice 1. Continuação...

\begin{tabular}{|c|c|c|}
\hline Táxon superior Espécie & Citação bibliográfica e/ou Coleção Científica & $\begin{array}{l}\text { Localidade- } \\
\text { tipo Araçá }\end{array}$ \\
\hline Lissomyema exilii (F. Müller, 1883) & $\begin{array}{l}\text { Ditadi, 1969a,b, 1970a,b,c, 1982b, 1994, 1996; Jorge \& Ditadi, } \\
\text { 1969; Jorge et al. 1969a; Amor, 1971; Macha \& Ditadi, 1972; } \\
\text { Anker et al. } 2005\end{array}$ & - \\
\hline Ochetostoma erythrogrammon Leuckart \& Rüppell, 1828 & Ditadi, 1983b; Anker et al. 2005 & - \\
\hline SIPUNCULA & & - \\
\hline Nephasoma confusum (Sluiter, 1902) & $\begin{array}{l}\text { Morgado, 1980b; Ditadi \& Migotto, 1981; Nalesso et al. 1995; } \\
\text { Morgado \& Tanaka, } 2001\end{array}$ & - \\
\hline Phascolosoma sp. & Nalesso et al. 1995 & - \\
\hline Sipunculus multisulcatus W. Fischer, 1913 & Jorge et al. 1970 & - \\
\hline Sipunculus nudus Linnaeus, 1766 & $\begin{array}{l}\text { Jorge et al. 1970; Ditadi, 1982a; Dworschak \& Rodrigues, } \\
\text { 1997; Kawauchi, 2005; Kihara et al. } 2007\end{array}$ & - \\
\hline Sipunculus phalloides (Pallas, 1774) & $\begin{array}{l}\text { Ditadi, 1982a; Kawauchi, 2005; Björnberg \& Kawauchi, 2006; } \\
\text { Kihara et al. } 2007\end{array}$ & - \\
\hline Sipunculus polymyotus Fisher, 1947 & Ditadi, 1982a (como Sipunculus natans) & - \\
\hline Sipunculus sp. & Jorge et al. 1969b; Kawauchi, 2005 & - \\
\hline Themiste alutacea (Grube \& Oersted, 1858) & Nalesso et al. 1995 & - \\
\hline Thysanocardia catharinae (Grube, 1868) & Arasaki, 1997; Pires Vanin et al. 1997 & - \\
\hline Xenosiphon branchiatus (Fischer, 1895) & Kawauchi, 2005 & - \\
\hline PYCNOGONIDA & & - \\
\hline Ammothella sp. & Duarte \& Nalesso, 1996 & - \\
\hline CRUSTACEA, STOMATOPODA & & - \\
\hline Acanthosquilla digueti (Coutière, 1905) & Rodrigues, 1966, 1971; Gomes-Corrêa, 1986 & - \\
\hline Alima hieroglyphica (Kemp, 1911) & Gomes-Corrêa, 1986 & - \\
\hline Coronis scolopendra Latreille, 1828 & Gomes-Corrêa, 1986 & - \\
\hline Erichthonius brasiliensis (Dana, 1853) & Nalesso et al. 1995 & - \\
\hline Lysiosquilla glabriuscula (Lamarck, 1818) & Gomes-Corrêa, 1986 & - \\
\hline Lysiosquilla scabricauda (Lamarck, 1818) & Dworschak \& Rodrigues, 1997 & - \\
\hline Padochela riisei Stimpson, 1860 & Nalesso et al. 1995 & - \\
\hline CRUSTACEA, DECAPODA & & - \\
\hline Albunea paretii Guérin-Méneville, 1853 & Rodrigues, 1971 & - \\
\hline Alpheus armillatus H. Milne Edwards, 1837 & Christoffersen, 1980 & - \\
\hline Alpheus bouvieri A. Milne-Edwards, 1878 & Christoffersen, 1980 & - \\
\hline Alpheus normanni Kingsley, 1878 & Christoffersen, 1980 & - \\
\hline Alpheus nuttingi (Schmitt, 1924) & Christoffersen, 1980 & - \\
\hline Alpheus sp. & Nalesso et al. 1995 & - \\
\hline Ambidexter symmetricus Manning \& Chace, 1971 & Christoffersen, 1980 & - \\
\hline Armases rubripes (Rathbun, 1897) & Prado, 1999 & - \\
\hline Automate evermanni Rathbun, 1901 & Dworschak \& Coelho, 1999 & - \\
\hline Axianassa australis Rodrigues \& Shimizu, 1992 & $\begin{array}{l}\text { Rodrigues \& Shimizu, } 1987 \text { (como Axianassa sp.); Rodrigues } \\
\text { \& Shimizu, 1992; Rodrigues et al. 1995; Dworschak \& Rod- } \\
\text { rigues, 1997; Dworschak \& Coelho, 1999; Coelho \& Rodrigues, } \\
\text { 2001; Kihara et al. } 2005\end{array}$ & - \\
\hline Calcinus tibicen (Herbst, 1791) & Biagi \& Mantelatto, 2005 & - \\
\hline Callianassa delicatula (Rodrigues \& Manning, 1992) & $\begin{array}{l}\text { Rodrigues \& Manning, } 1992 \text { (como Biffarius delicatulus); } \\
\text { Sakai, } 1999\end{array}$ & - \\
\hline Callichirus major (Say, 1818) & Rodrigues, 1971, 1983; Dworschak \& Coelho, 1999 & - \\
\hline
\end{tabular}

HIBSP - Herbário Científico Maria Eneyda P. K. Fidalgo do Instituto de Botânica de São Paulo

MNRJ - Museu Nacional do Rio de Janeiro

MZUSP - Museu de Zoologia da Universidade de São Paulo

SPFUSP - Coleção de Algas do Departamento de Botânica da Universidade de São Paulo

USNM - National Museum of Natural History, Smithsonian Institution, USA 
Apêndice 1. Continuação...

\begin{tabular}{|c|c|c|}
\hline Táxon superior Espécie & Citação bibliográfica e/ou Coleção Científica & $\begin{array}{l}\text { Localidade- } \\
\text { tipo Araçá }\end{array}$ \\
\hline Callichirus sp. & Kihara \& Rocha, 1993 & - \\
\hline Callinectes danae Smith, 1869 & $\begin{array}{l}\text { Lacerda, 1981; Mossolin, 1994; Caprara \& Freitas, 1996; Lo- } \\
\text { wenthal et al. 1996; Nucci, 1998; Genofre et al. 2000; Nucci } \\
\text { et al. 2001; Turra \& Leite, 2005; Padovanni, } 2009\end{array}$ & - \\
\hline Callinectes ornatus Ordway, 1863 & Nucci, 1998; Nucci et al. 2001; Gorni \& Weber, 2004 & - \\
\hline Clibanarius antillensis Stimpson, 1862 & $\begin{array}{l}\text { Garcia, 1979; Brossi-Garcia \& Hebling, 1983; Arantes \& Leite, } \\
\text { 1991b, 1995; Jacobi, 1992; Leite et al. 1995, 1996, 1998; } \\
\text { Nalesso et al. 1995; Turra \& Leite, 1995, 1996, 2000, 2001, } \\
2002 \text { 2004, 2007; Turra et al. 1995, 1999, 2000; Barata et al. } \\
\text { 1996; Castelo-Branco et al. 1996; Jacobucci \& Leite, 1996; } \\
\text { Turra \& Denadai, 2001, 2002, 2003, 2004; Souza, 2003; Turra, } \\
\text { 2003, 2004, 2005 }\end{array}$ & - \\
\hline Clibanarius sclopetarius (Herbst,1796) & $\begin{array}{l}\text { Brossi-Garcia, 1987;Arantes \& Leite, 1991b, 1995; Jacobi, } \\
\text { 1992; Leite et al. 1995, 1996, 1998; Turra \& Leite, 1995, 1996, } \\
\text { 2000, 2001, 2002, 2007; Turra et al. 1995, 1999, 2000; Barata } \\
\text { et al. 1996; Castelo-Branco et al. 1996; Jacobucci \& Leite, } \\
\text { 1996; Turra, 2003, 2004, 2005; Turra \& Denadai, 2001, 2002, } \\
\text { 2003; Turra \& Leite, 2004 }\end{array}$ & - \\
\hline Clibanarius vitattus (Bosc, 1802) & $\begin{array}{l}\text { Brossi-Garcia, 1988; Arantes \& Leite, 1989, 1991b, 1994, } \\
\text { 1995; Jacobi, 1992; Arantes, 1994; Leite et al. 1995, 1996, } \\
\text { 1998; Turra \& Leite, 1995, 1996, 2000, 2001; Turra et al. } \\
\text { 1995, 1999, 2000; Barata et al. 1996; Castelo-Branco et al. } \\
\text { 1996; Jacobucci \& Leite, 1996, 1998a; Nucci, 1998; Leite \& } \\
\text { Jacobucci, 1998; Nucci et al. 2001; Turra \& Denadai, 2001, } \\
\text { 2002, 2003; Souza, 2003; Turra, 2003, 2004, 2005; Turra \& } \\
\text { Leite, 2002, 2004, 2007; Biagi \& Mantelatto, 2005; Gonçalves } \\
\text { et al. 2006; Turra, 2007 }\end{array}$ & - \\
\hline Clibanarius sp. & $\begin{array}{l}\text { Arantes \& Leite, 1991a; Turra \& Leite, 1995, 1996, 1997; } \\
\text { Barata, 1996; Barata et al. } 1996\end{array}$ & - \\
\hline Epialtus brasiliensis Dana, 1852 & $\begin{array}{l}\text { Duarte, 1980b; Nalesso et al. 1995; Duarte \& Nalesso, 1996; } \\
\text { Morgado \& Tanaka, } 2001\end{array}$ & - \\
\hline Epialtus sp. & Milanelli, 2003 & - \\
\hline Eriphia gonagra (Fabricius, 1781) & Santos \& Bueno, 2001; Turra \& Leite, 2005 & - \\
\hline Eurypanopeus abbreviatus (Stimpson, 1860) & Milanelli, 2003 & - \\
\hline Hexapanopeus paulensis Rathbun, 1930 & Duarte, 1980b; Nalesso et al. 1995; Duarte \& Nalesso, 1996 & - \\
\hline Hexapanopeus schmitti Rathbun, 1930 & $\begin{array}{l}\text { Duarte, 1980b; Duarte \& Nalesso, 1996; Nucci, 1998; Nucci } \\
\text { et al. 2001; Amaral et al. } 2003\end{array}$ & - \\
\hline Hippolyte curacaoensis Schmitt, 1924 & Christoffersen, 1980 & - \\
\hline Lepidopa sp. & Ditadi, $1982 b$ & - \\
\hline Leptalpheus axianassae Dworschak \& Coelho, 1999 & Dworschak \& Coelho, 1999 & - \\
\hline Megalobrachium soriatum (Say, 1818) & $\begin{array}{l}\text { Duarte, 1980b; Morgado, 1980b; Nalesso et al. 1995; Duarte } \\
\text { \& Nalesso, 1996; Morgado \& Tanaka, } 2001\end{array}$ & - \\
\hline Menippe nodifrons Stimpson, 1859 & $\begin{array}{l}\text { Duarte, 1980b; Nalesso et al. 1995; Duarte \& Nalesso, 1996; } \\
\text { Turra \& Leite, } 2005\end{array}$ & - \\
\hline Microphrys bicornutus (Latreille, 1825) & Duarte, 1980b; Nalesso et al. 1995; Duarte \& Nalesso, 1996 & - \\
\hline Mithraculus forceps (A. Milne-Edwards, 1875) & $\begin{array}{l}\text { Morgado, 1980b (como Mithrax mitraculus forceps); Morgado } \\
\text { \& Tanaka, } 2001\end{array}$ & - \\
\hline
\end{tabular}

HIBSP - Herbário Científico Maria Eneyda P. K. Fidalgo do Instituto de Botânica de São Paulo

MNRJ - Museu Nacional do Rio de Janeiro

MZUSP - Museu de Zoologia da Universidade de São Paulo

SPFUSP - Coleção de Algas do Departamento de Botânica da Universidade de São Paulo

USNM - National Museum of Natural History, Smithsonian Institution, USA 
Apêndice 1. Continuação...

\begin{tabular}{|c|c|c|}
\hline Táxon superior Espécie & Citação bibliográfica e/ou Coleção Científica & $\begin{array}{l}\text { Localidade- } \\
\text { tipo Araçá }\end{array}$ \\
\hline Pachycheles rugimanus Milne-Edwards, 1880 & $\begin{array}{l}\text { Duarte, 1980b; Morgado, 1980b (como Pachycheles } \\
\text { maginanus); Nalesso et al. 1995; Duarte \& Nalesso, 1996; } \\
\text { Morgado \& Tanaka, } 2001\end{array}$ & - \\
\hline Pachygrapsus gracilis (de Saussure, 1858) & Prado, 1999 & - \\
\hline Pachygrapsus transversus (Gibbes, 1850) & Duarte, 1980b; Duarte \& Nalesso, 1996; Milanelli, 2003 & - \\
\hline Pagurus criniticornis (Dana, 1852) & $\begin{array}{l}\text { Moreira et al. 1978, 1979; Garcia, 1979; Vernberg et al. 1981; } \\
\text { Nipper-Buscariolli \& Moreira, 1982, 1983; Nipper-Buscariolli, } \\
\text { 1983; Blaszkowski, C. \& Moreira. G.S. 1986; Barrios \& Freitas, } \\
\text { 1987; Arantes \& Leite, 1989, 1991b; Oishi, 1994; Oishi } \\
\text { \& Leite, 1994; Arantes \& Leite, 1995; Gabriel \& Moreira, } \\
\text { 1995b; Turra et al. 1995, 1999, 2000; Araújo \& Leite, 1996; } \\
\text { Castelo-Branco et al. 1996; Leite et al. 1996, 1998; Nucci, } \\
\text { 1998; Nucci et al. 2001; Souza et al. 2001; Souza \& Leite, 2002; } \\
\text { Amaral et al. 2003; Souza, 2003; Turra \& Denadai, 2001, 2003, } \\
\text { 2004; Turra, 2003, 2005; Turra \& Leite, 2003, 2007; Barros, } \\
\text { 2004; Mantelatto \& Fantucci, 2007; Fantucci \& Mantelatto, } \\
\text { 2008; Dominciano et al. 2009 }\end{array}$ & - \\
\hline Panopeus americanus de Saussure, 1857 & $\begin{array}{l}\text { Vergamini \& Mantelatto, 2005, 2006c, 2007, 2008a,b; } \\
\text { Vergamini, 2006; Iguchi, 2008; Iguchi et al. } 2008\end{array}$ & - \\
\hline Panopeus austrobesus Williams, 1983 & Nucci, 1998; Nucci et al. 2001 & - \\
\hline Panopeus occidentalis de Saussure, 1857 & $\begin{array}{l}\text { Turra \& Leite, 2005; Vasconcelos et al. 2007, 2008; Vasconcelos, } \\
\text { 2008; Vasconcelos \& Mantelatto, 2008; Padovanni, } 2009\end{array}$ & - \\
\hline Panopeus sp. & Santos \& Bueno, 2001; Vergamini \& Mantelatto, 2006a,b & - \\
\hline Penaeus schmitti Burkenroad, 1936 & Blumer \& Moreira, 1995; Gabriel \& Moreira, 1995a & - \\
\hline Petrolisthes armatus (Gibbes, 1850) & $\begin{array}{l}\text { Santos \& Bueno, 2001; Miranda \& Mantelatto, 2006a,b, 2008, } \\
\text { prelo-a,b; Silva \& Mantelatto, 2006, 2008a,b; ; Wehrtman } \\
\text { et al. 2006; Silva, 2007; Wehrtman et al. 2007; Wehrtman } \\
\text { et al. prelo }\end{array}$ & - \\
\hline Petrolisthes galathinus (Bosc, 1802) & $\begin{array}{l}\text { Duarte, 1980b; Morgado, 1980b; Duarte \& Morgado, 1983; } \\
\text { Nalesso et al. 1995; Duarte \& Nalesso, 1996; Morgado \& } \\
\text { Tanaka, } 2001\end{array}$ & - \\
\hline Pilumnus dasypodus Kingsley, 1879 & $\begin{array}{l}\text { Duarte, 1980b; Morgado, 1980b; Duarte \& Morgado, 1983; } \\
\text { Nalesso et al. 1995; Duarte \& Nalesso, 1996; Morgado \& } \\
\text { Tanaka, } 2001\end{array}$ & - \\
\hline Pilumnus floridanus Stimpson, 1871 & Duarte, 1980b; Nalesso et al. 1995; Duarte \& Nalesso, 1996 & - \\
\hline Pinnixa sayana Stimpson, 1860 & Arasaki, 1997 & - \\
\hline Pinnixa sp. & Ditadi, 1970a; Ditadi, 1982b; Anker et al. 2005 & - \\
\hline Planes cyaneus Dana, 1852 & Prado \& Melo, 2002 & - \\
\hline Podochela riisei Stimpson, 1860 & $\begin{array}{l}\text { Duarte, 1980b; Morgado, 1980b; Duarte \& Nalesso, 1996; } \\
\text { Morgado \& Tanaka, } 2001\end{array}$ & - \\
\hline Porcellana sp. & Duarte \& Nalesso, 1996 & - \\
\hline PORCELLANIDAE SP. & Duarte, 1980b; Morgado, 1980b; Morgado \& Tanaka, 2001 & - \\
\hline Sergio guassutinga (Rodrigues, 1971) & $\begin{array}{l}\text { Rodrigues, } 1971 \text { (como Callianassa guassutinga) ; Manning } \\
\text { \& Lemaitre, } 1993\end{array}$ & - \\
\hline Synalpheus apioceros Coutière, 1909 & $\begin{array}{l}\text { Christoffersen, 1980; Duarte, 1980b; Morgado, 1980b; Nalesso } \\
\text { et al. 1995; Duarte \& Nalesso, 1996; Morgado \& Tanaka, } \\
2001\end{array}$ & - \\
\hline
\end{tabular}

HIBSP - Herbário Científico Maria Eneyda P. K. Fidalgo do Instituto de Botânica de São Paulo

MNRJ - Museu Nacional do Rio de Janeiro

MZUSP - Museu de Zoologia da Universidade de São Paulo

SPFUSP - Coleção de Algas do Departamento de Botânica da Universidade de São Paulo

USNM - National Museum of Natural History, Smithsonian Institution, USA 
Apêndice 1. Continuação...

\begin{tabular}{|c|c|c|}
\hline Táxon superior Espécie & Citação bibliográfica e/ou Coleção Científica & $\begin{array}{l}\text { Localidade- } \\
\text { tipo Araçá }\end{array}$ \\
\hline Synalpheus brevicarpus (Herrick, 1891) & $\begin{array}{l}\text { Christoffersen, 1980; Duarte, 1980b; Morgado, 1980b; Duarte } \\
\text { \& Morgado, 1983; Nalesso et al. 1995; Duarte \& Nalesso, 1996; } \\
\text { Morgado \& Tanaka, } 2001\end{array}$ & - \\
\hline Synalpheus fritzmuelleri Coutière, 1909 & $\begin{array}{l}\text { Christoffersen, 1980; Duarte, 1980b; Morgado, 1980b; Nalesso } \\
\text { et al. 1995; Duarte \& Nalesso, 1996; Morgado \& Tanaka, } \\
2001\end{array}$ & - \\
\hline Thor manningi Chace, 1972 & $\begin{array}{l}\text { Duarte, 1980b; Duarte \& Nalesso, 1996; Morgado \& Tanaka, } \\
2001\end{array}$ & - \\
\hline Typton gnathophylloides Holthuis, 1951 & Duarte, 1980b; Nalesso et al. 1995; Duarte \& Nalesso, 1996 & - \\
\hline Uca leptodactyla Rathbun, 1898 & MZUSP & - \\
\hline Uca maracoani Latreille, 1802-1803 & MZUSP & - \\
\hline Uca rapax (Smith, 1870) & $\begin{array}{l}\text { Genofre Neto, 1972; Marques, 1972; Marques \& Sawaya, 1972; } \\
\text { Sawaya \& Marques, 1972; McNamara \& Moreira, } 1983\end{array}$ & - \\
\hline Uca thayeri Rathbun, 1900 & Ditadi, 1982a; Nucci, 1998; Nucci et al. 2001; MZUSP & - \\
\hline Uca uruguayensis Nobili, 1901 & McNamara \& Moreira, 1983; MZUSP & - \\
\hline Uca sp. & Sawaya \& Genofre, 1972 & - \\
\hline Upogebia affinis (Say, 1818) & Ditadi, $1982 b$ & - \\
\hline Upogebia inomissa Williams, 1993 & Nucci, 1998; Nucci et al. 2001 & - \\
\hline Upogebia omissa Gomes Corrêa, 1968 & Nucci, 1998; Nucci et al. 2001 & - \\
\hline Upogebia paraffinis Williams, 1993 & Dworschak \& Coelho, 1999 & - \\
\hline Xiphopenaeus kroyeri (Heller, 1862) & Pires-Vanin et al. 1997 & - \\
\hline CRUSTACEA, AMPHIPODA & & - \\
\hline Amphithoe ramondi Audouin, 1826 & Nalesso et al. 1995 & - \\
\hline Caprella scaura Templeton, 1836 & Nalesso et al. 1995 & - \\
\hline Corophium sp. & Ditadi, 1982b & - \\
\hline Cymadusa filosa Savigny, 1816 & Nalesso et al. 1995 & - \\
\hline Elasmopus rapax Costa, 1853 & Nalesso et al. 1995 & - \\
\hline Hyale media (Dana, 1853) & Nalesso et al. 1995 & - \\
\hline Lembos sp. & Nalesso et al. 1995 & - \\
\hline Leucothoe alata JL Barnard, 1959 & Nalesso et al. 1995 & - \\
\hline Leucothoe spinicarpa (Abildgaard, 1789) & Nalesso et al. 1995 & - \\
\hline Lysianassa sp. & Nalesso et al. 1995 & - \\
\hline Phoxocephalopsis zimmeri (Schellenberg, 1931) & Arasaki, 1997; Pires-Vanin et al. 1997 & - \\
\hline CRUSTACEA, TANAIDACEA & & - \\
\hline Kalliapseudes schubarti Mañé-Garzon, 1949 & $\begin{array}{l}\text { Moreira, 1972a,b;Leite \& Ferreira, 1988; Leite, 1989b, 1991a, } \\
\text { 1995; Agujaro \& Leite, 1991; Soto-Espinosa, 1993; Ramos, } \\
\text { 1994; Agujaro, 1996; Nucci et al. 1997; Nucci, 1998; Leite } \\
\text { et al. 1992, 2003; Souza \& Amaral, 1998; Souza et al. 1998; } \\
\text { Nucci et al. 2001; Amaral et al. 2003; Padovanni, } 2009\end{array}$ & - \\
\hline Kalliapseudes sp. & Dworschak \& Rodrigues, 1997 & - \\
\hline Leptochelia savignyi (Kroyer, 1842) & Nalesso et al. 1995 & - \\
\hline CRUSTACEA, ISOPODA & & - \\
\hline Apanthura sp. & Arasaki, 1997; Pires-Vanin et al. 1997 & - \\
\hline Aporobopyrus curtatus (Richardson, 1904) & $\begin{array}{l}\text { Duarte \& Morgado, 1983; Santos \& Bueno, 2001; Miranda \& } \\
\text { Mantelatto, prelo-b }\end{array}$ & - \\
\hline Bopyrella harmopleon Bowman, 1956 & Duarte \& Morgado, 1983 & - \\
\hline
\end{tabular}

HIBSP - Herbário Científico Maria Eneyda P. K. Fidalgo do Instituto de Botânica de São Paulo

MNRJ - Museu Nacional do Rio de Janeiro

MZUSP - Museu de Zoologia da Universidade de São Paulo

SPFUSP - Coleção de Algas do Departamento de Botânica da Universidade de São Paulo

USNM - National Museum of Natural History, Smithsonian Institution, USA 
Apêndice 1. Continuação...

\begin{tabular}{|c|c|c|}
\hline Táxon superior Espécie & Citação bibliográfica e/ou Coleção Científica & $\begin{array}{l}\text { Localidade- } \\
\text { tipo Araçá }\end{array}$ \\
\hline Cirolana parva Hansen, 1890 & Nalesso et al. 1995 & - \\
\hline Cyathura sp. & Nalesso et al. 1995 & - \\
\hline Erichsonella filiformis (Say, 1818) & Nalesso et al. 1995 & - \\
\hline Excirolana sp. & Amaral et al. 1990 & - \\
\hline Excorallana quadricornis (Hansen, 1890) & $\begin{array}{l}\text { Duarte, 1980b; Duarte \& Nalesso, 1996; Morgado \& Tanaka, } \\
2001\end{array}$ & - \\
\hline Ligia exotica Roux, 1828 & Milanelli, 2003 & - \\
\hline Paracerceis sculpta (Holmes, 1904) & Nalesso et al. 1995 & - \\
\hline CRUSTACEA, COPEPODA & & - \\
\hline Acartia lilljeborgi Giesbrecht, 1892 & Gaeta et al. 1987 & - \\
\hline $\begin{array}{l}\text { Carcinocirrus minipedia (gen.sp.nov.) Björnberg \& Santos } \\
\text { (submetido) }\end{array}$ & Björnberg \& Santos (submetido) & - \\
\hline Catinia aiso Kihara, Rocha \& Santos, 2005 & Kihara et al. 2005 & - \\
\hline Catinia rosea Björnberg \& Kawauchi, 2006 & Björnberg \& Kawauchi, 2006 & - \\
\hline Hemicyclops sp. (2 sp. nov.) & Kihara, \& Rocha, 1993 & - \\
\hline $\begin{array}{l}\text { Myzomolgus sipunculensis Kihara; Björnberg \& Kawauchi } \\
\text { (2007) }\end{array}$ & Kihara et al. 2007 & - \\
\hline Thespesiopsyllus sp. & Duarte \& Morgado, 1983 & - \\
\hline CRUSTACEA, CIRRIPEDIA & & - \\
\hline Balanus sp. & Milanelli, 2003 & - \\
\hline Chthamalus bisinuatus Pilsbry, 1916 & Milanelli, 2003 & - \\
\hline Megabalanus coccopoma (Darwin, 1854) & Milanelli, 2003 & - \\
\hline Sacculina hirsuta Boschma, 1925 & Young, 1987 & - \\
\hline Tetraclita stalactifera (Lamarck, 1818) & Milanelli, 2003 & - \\
\hline KAMPTOZOA (ENTOPROCTA) & & - \\
\hline Barentsia discreta (Busk, 1886) & MZUSP & - \\
\hline Loxosomella ditadii Marcus \& Marcus, 1968 & Marcus \& Marcus, 1968; Ditadi, 1970a; Anker et al. 2005 & - \\
\hline Loxosomella zima Marcus \& Marcus, 1968 & Marcus \& Marcus, 1968; Ditadi, 1970a; Anker et al. 2005 & - \\
\hline Pedicellina cernua (Pallas, 1774) & MZUSP & - \\
\hline BRYOZOA (ECTOPROCTA) & & - \\
\hline Aetea anguina (Linnaeus, 1758) & MZUSP & - \\
\hline Aetea ligulata (Busk, 1852) & MZUSP & - \\
\hline Amathia distans Busk, 1886 & MZUSP & - \\
\hline Amathia vidovici (Heller, 1867) & MZUSP & - \\
\hline Antropora leucocypha (Marcus, 1937) & MZUSP & - \\
\hline Arthropoma cecilii (Audouin, 1826) & MZUSP & - \\
\hline Beania klugei Cook, 1968 & MZUSP & - \\
\hline Beania mirabilis Johnston, 1840 & MZUSP & - \\
\hline Biflustra denticulata (Busk, 1856) & MZUSP & - \\
\hline Bowerbankia maxima Winston, 1982 & MZUSP & - \\
\hline Bowerbankia sp.1 & MZUSP & - \\
\hline Bugula neritina (Linnaeus, 1758) & MZUSP & - \\
\hline Bugula sp. & Milanelli, 2003 & - \\
\hline Bugula stolonifera Ryland, 1960 & MZUSP & - \\
\hline Catenicella contei (Audouin, 1826) & Milanelli, 2003; MZUSP & - \\
\hline \multicolumn{3}{|c|}{ HIBSP - Herbário Científico Maria Eneyda P. K. Fidalgo do Instituto de Botânica de São Paulo } \\
\hline \multicolumn{3}{|c|}{ MNRJ - Museu Nacional do Rio de Janeiro } \\
\hline \multicolumn{3}{|l|}{ MZUSP - Museu de Zoologia da Universidade de São Paulo } \\
\hline SPFUSP - Coleção de Algas do Departamento de Botânica da Unive & ersidade de São Paulo & \\
\hline
\end{tabular}


Apêndice 1. Continuação...

\begin{tabular}{|c|c|c|}
\hline Táxon superior Espécie & Citação bibliográfica e/ou Coleção Científica & $\begin{array}{l}\text { Localidade- } \\
\text { tipo Araçá }\end{array}$ \\
\hline Catenicella uberrima (Harmer, 1957) & MZUSP & - \\
\hline Caulibugula armata Verrill, 1900 & Milanelli, 2003 & - \\
\hline Celleporaria mordax (Marcus, 1937) & MZUSP & - \\
\hline Crisia pseudosolena (Marcus, 1937) & MZUSP & - \\
\hline Electra bellula (Hincks, 1881) & MZUSP & - \\
\hline Electra tenella (Hincks, 1880) & MZUSP & - \\
\hline Hippoporella gorgonensis Hastings, 1930 & MZUSP & - \\
\hline Hippothoa brasiliensis Morris, 1980 & Milanelli, 2003 (como Hippothoa hyalina) & - \\
\hline Nellia oculata Busk, 1852 & MZUSP & - \\
\hline Nolella aff. stipata Gosse, 1855 & MZUSP & - \\
\hline Parasmittina sp.1 & MZUSP & - \\
\hline Parasmittina sp.2 & MZUSP & - \\
\hline Rhynchozoon verruculatum (Smitt, 1873) & MZUSP & - \\
\hline Savignyella lafontii (Audouin \& Savigny, 1826) & Milanelli, 2003; MZUSP & - \\
\hline Schizoporella errata (Waters, 1878) & $\begin{array}{l}\text { Morgado \& Duarte, 1979; Morgado, 1980a,b, 1984, 1985; } \\
\text { Duarte \& Morgado, 1983; Morgado \& Amaral, 1981d, } \\
\text { 1983, 1983, 1984a,b, 1985; Duarte \& Nalesso, } 1996 \text { (como } \\
\text { Schizoporella unicornis); Morgado \& Tanaka, 2001; } \\
\text { Milanelli, } 2003\end{array}$ & - \\
\hline Schizoporella pungens Canu \& Bassler, 1928 & MZUSP & - \\
\hline Scrupocellaria bertholletii (Audouin, 1826) & MZUSP & - \\
\hline Scrupocellaria frondis Kirkpatrick, 1890 & MZUSP & - \\
\hline Scrupocellaria regularis (Osburn, 1940) & MZUSP & - \\
\hline Scrupocellaria sp. & MZUSP & - \\
\hline Siniopelta costazii (Audouin), 1826 & Milanelli, 2003 & - \\
\hline Sundanella sp. & MZUSP & - \\
\hline Synnotum aegyptiacum (Audouin, 1826) & MZUSP & - \\
\hline Watersipora subtorquata (d’Orbigny, 1842) & MZUSP & - \\
\hline Zoobotryon verticillatum Delle Chiaje, 1822 & Migotto, 1993 (como Zoobotryon sp.); MZUSP & - \\
\hline ECHINODERMATA, ASTEROIDEA & & - \\
\hline Asterina stellifera (Möbius, 1859) & Dolder, 1973 & - \\
\hline Astropecten brasiliensis Müller \& Troschel, 1842 & Netto, 2006 & - \\
\hline Astropecten marginatus Gray, 1840 & Netto, 2006 & - \\
\hline Luidia clathrata (Say, 1825) & Netto, 2006 & - \\
\hline Luidia senegalensis (Lamark, 1816) & Netto, 2006 & - \\
\hline ECHINODERMATA, OPHIRUROIDEA & & - \\
\hline Amphipholis squamata (Delle Chiaje, 1828) & $\begin{array}{l}\text { Milanelli, } 2003 \text { (como Amphipholis squamata); Duarte, 1980b; } \\
\text { Nalesso et al. 1995; Duarte \& Nalesso, 1996; Morgado \& } \\
\text { Tanaka, } 2001 \text { (como Axiognathus squamatus) }\end{array}$ & - \\
\hline Hemipholis elongata (Say, 1825) & Netto, 2006 & - \\
\hline Ophiactis lymani Ljungman, 1872 & $\begin{array}{l}\text { Duarte, 1980b; Duarte \& Morgado, 1983; Nalesso et al. 1995; } \\
\text { Duarte \& Nalesso, 1996; Morgado \& Tanaka, } 2001\end{array}$ & - \\
\hline Ophiactis savignyi (Müller \& Troschel, 1842) & $\begin{array}{l}\text { Duarte, 1980a,b; Morgado, 1980b; Duarte \& Morgado, 1983; } \\
\text { Nalesso et al. 1995; Duarte \& Nalesso, 1996; Morgado \& } \\
\text { Tanaka, } 2001\end{array}$ & - \\
\hline
\end{tabular}

HIBSP - Herbário Científico Maria Eneyda P. K. Fidalgo do Instituto de Botânica de São Paulo

MNRJ - Museu Nacional do Rio de Janeiro

MZUSP - Museu de Zoologia da Universidade de São Paulo

SPFUSP - Coleção de Algas do Departamento de Botânica da Universidade de São Paulo

USNM - National Museum of Natural History, Smithsonian Institution, USA 
Apêndice 1. Continuação...

\begin{tabular}{|c|c|c|}
\hline Táxon superior Espécie & Citação bibliográfica e/ou Coleção Científica & $\begin{array}{c}\text { Localidade- } \\
\text { tipo Araçá }\end{array}$ \\
\hline Ophiothrix (Opiothrix) angulata Say, 1825 & $\begin{array}{l}\text { Duarte, 1980b; Morgado, 1980b; Nalesso et al. 1995; Duarte } \\
\text { \& Nalesso, 1996; Morgado \& Tanaka, 2001; Milanelli, } 2003 \\
\text { (como Ophiothrix angulata) }\end{array}$ & - \\
\hline ECHINODERMATA, ECHINOIDEA & & - \\
\hline Arbacia lixula Linnaeus, 1758 & Milanelli, 2003 & - \\
\hline Echiometra lucunter (Linnaeus, 1758) & Milanelli, 2003 & - \\
\hline Encope emarginata (Leske 1778) & MZUSP & - \\
\hline Lytechinus variegatus Lamarck, 1816 & Milanelli, 2003 & - \\
\hline ECHINODERMATA, HOLOTHUROIDEA & & - \\
\hline Holothuria grisea Selenka, 1867 & $\begin{array}{l}\text { Migotto, 1993; Nalesso et al. } 1995 \text { (como Ludwigothuria } \\
\text { grisea); Milanelli, } 2003\end{array}$ & - \\
\hline Pentacta peterseni Lopez, 1969 & Dolder, 1973 & - \\
\hline Synaptula hidriformis (Lesueur, 1824) & Nalesso et al. 1995 & - \\
\hline ECHINODERMATA, CRINOIDEA & & - \\
\hline Tropiometra carinata (Lamarck, 1816) & Milanelli, 2003 & - \\
\hline HEMICHORDATA, ENTEROPNEUSTA & & - \\
\hline Balanoglossus clavigerus delle Chiaje, 1829 & Burdon-Jones \& Petersen, 1964 & - \\
\hline Balanoglossus gigas F. Müller in Spengel, 1893 & $\begin{array}{l}\text { Burdon-Jones \& Petersen, 1964; Sawaya, 1950, 1951; Rosa, } \\
1973\end{array}$ & - \\
\hline Glossobalanus crozieri van der Horst, 1924 & Petersen \& Ditadi, 1971 & - \\
\hline Willeyia loya Petersen, 1965 & $\begin{array}{l}\text { Burdon-Jones \& Petersen, } 1964 \text { (como Willeyia sp.); Petersen, } \\
1965\end{array}$ & $\mathrm{X}$ \\
\hline UROCHORDATA, ASCIDIACEA & & - \\
\hline Ascidia sydneiensis (Stimpson, 1855) & Rodrigues, 1962 & - \\
\hline Botrylloides giganteum (Pérès, 1949) & USNM & - \\
\hline Botrylloides nigrum Herdman, 1886 & Rodrigues, 1962; Rodrigues \& Rocha, 1993 & - \\
\hline Botryllus tabori Rodrigues, 1962 & Rodrigues, 1962; Rodrigues \& Rocha, 1993 & $\mathrm{X}$ \\
\hline Clavelina oblonga Herdmann, 1880 & Rodrigues, 1962; Rodrigues \& Rocha, 1993; Milanelli, 2003 & - \\
\hline Didemnum psammathodes (Sluiter, 1895) & Rodrigues \& Rocha, 1993; Milanelli, 2003 & - \\
\hline Didemnum speciosum (Herdman, 1886) & Rodrigues \& Rocha, 1993 & - \\
\hline Didemnum vanderhorst Van Name 1924 & Rocha \& Monniot, 1995; Milanelli, 2003 & - \\
\hline Diplosoma listerianum (Milne-Edwards, 1841) & Rodrigues \& Rocha, 1993; Milanelli, 2003 & - \\
\hline Distaplia bermudensis Van Name, 1902 & Milanelli, 2003 & - \\
\hline Herdmania pallida (Heller, 1878) & Milanelli, 2003 (como H. momus) & - \\
\hline Microcosmus exasperatus Heller, 1878 & Rodrigues, 1962 & - \\
\hline Polyandrocarpa zorritensis (Van Name, 1931) & Rodrigues, 1962; Milanelli, 2003 & - \\
\hline Polyclinum constellatum Savigny, 1816 & Rodrigues \& Rocha, 1993 & - \\
\hline Styela plicata (Lesueur, 1823) & Rodrigues, 1962 & - \\
\hline Symplegma brakenhielmi (Michaelsen, 1904) & $\begin{array}{l}\text { Rodrigues, 1962; Rodrigues \& Rocha, 1993; Milanelli, } 2003 \\
\text { (como Symplegma viride Herdman, 1886) }\end{array}$ & - \\
\hline Trididemnum orbiculatum (Van Name, 1902) & Rodrigues \& Rocha, 1993 & - \\
\hline \multicolumn{3}{|c|}{ HIBSP - Herbário Científico Maria Eneyda P. K. Fidalgo do Instituto de Botânica de São Paulo } \\
\hline \multicolumn{3}{|c|}{ MNRJ - Museu Nacional do Rio de Janeiro } \\
\hline \multicolumn{3}{|l|}{ MZUSP - Museu de Zoologia da Universidade de São Paulo } \\
\hline SPFUSP - Coleção de Algas do Departamento de Botâ & rsidade de São Paulo & \\
\hline
\end{tabular}


Apêndice 2. Bibliografia produzida sobre a Baía do Araçá e adjacências. Appendix 2. Bibliography about Araçá Bay and adjacencies.

\section{Artigos em periódicos}

AMARAL, A.C.Z. \& MORGADO, E.H. 1994. Alteraciones en la fauna de anélidos poliquetos de Araçá, São Sebastião (SP - Brasil). Rev. Acad. Colomb. Cienc. 19(72):147-152.

AMARAL, A.C.Z., DENADAI, M.R., TURRA, A. \& RIZZO, A.E. 2003. Intertidal macrofauna in Brazilian subtropical sandy beaches landscape. J. Coastal Res. 35:446-455.

AMARAL, A.C.Z., MORGADO, E.H. \& SALVADOR, L.B. 1998. Poliquetas bioindicadores de poluição orgânica em praias paulistas. Rev. bras. biol. 58(2):307-316.

AMARAL, A.C.Z., MORGADO, E.H., LOPES, P.P., BELÚCIO, L.F., LEITE, F.P.P. \& FERREIRA, C.P. 1990. Composition and distribution of the intertidal macrofauna of sandy beaches on São Paulo coast. Publ. ACIESP 71(3):258-279.

AMOR, A. 1971. Echiura del Brasil. Physis 81(3):521-538.

ANKER, A., MURINA, G.V., LIRA, C, CARIPE, V., RICHARD PALMER, A. \& MING-SHIOU, J. 2005. Macrofauna associated with echiuran burrows: A review with new observations of the innkeeper worm, Ochetostoma erythrogrammon Leuckart and Rüppel, in Venezuela. Zool. Stud. 44(2):157-190.

ARRUDA, E.P. \& AMARAL, A.C.Z. 2003. Spatial distribution of mollusks in the intertidal zone of sheltered beaches in southeastern of Brazil. Rev. Bras. Zool. 20:291-300.

ARRUDA, E.P., DOMANESCHI, O. \& AMARAL, A.C.Z. 2003. Mollusc feeding guilds on sandy beaches in São Paulo State, Brazil. Mar. Biol. 143(4):691-701.

BJÖRNBERG, T.K.S. 1959. On Enteropneusta from Brazil. Bolm Inst. oceanogr. Univ. S. Paulo 10:1-104.

BJÖRNBERG, T.K.S. \& SANTOS, C. (submetido). Carcinocirrus minipedia n. gen. and sp. of Laophontidae (Copepoda, Crustacea), commensal on panopeid crabs. Nauplius.

BJÖRNBERG, T.K.S. \& KAWAUCHI, G.Y. 2006. Catinia rosea sp. nov. (Copepoda, Catiniidae) associated with Sipunculus phalloides (Pallas) from São Sebastião Channel, Brazil. Nauplius 14(2):83-88.

BLASZKOWSKI, C. \& MOREIRA. G.S. 1986. Combined effects of temperature and salinity on the survival and duration of larval stages of Pagurus criniticornis (Dana) (Crustacea, Paguridae). J. Exp. Mar. Biol. Ecol. 103:77-86.

BROSSI-GARCIA, A.L. 1987. Morphology of the larval stages of Clibanarius sclopetarius (Herbst, 1796) (Decapoda, Diogenidae) reared in the laboratory. Crustaceana 52(3):251-275.

BROSSI-GARCIA, A.L. 1988. Juvenile development of Clibanarius vittatus (Bosc, 1802) (Decapoda, Anomura), in the laboratory. Crustaceana 54(3): 294-313.

BROSSI-GARCIA, A.L. \& HEBLING, N.J. 1983a. Desenvolvimento pósembrionario de Clibanarius antillensis Stimpson, 1859 (Crustacea, Diogenidae), em laboratório. Bol. Zool. Univ. S. Paulo 6:89-111.

BURDON-JONES, C. \& PETERSEN, J.A. 1964. Another giant enteropneust from the Atlantic. Nature 203:97-98.

CARRERA-PARRA, L.F., RIZZO, A.E. \& SALAZAR-VALLEJO, S.I 2008. Redescription of Eunice sebastiani and recognition of E. riojai (Polychaeta: Eunicidae). J. Mar. Biol. Assoc. U. K. 88(3):503-507.

CARVALHO, M.S. \& HAJDU, E. 2001. Comments on brazilian Halicondria Fleming (Halichondriidae, Halichondrida, Demospongiae), with the description of four new species from São Sebastião Channel and its environs (Tropical Southwestern Atlantic). Rev. Bras. Zool. 18(supl.1):161-180.

COELHO, V.R. \& RODRIGUES, S.A. 2001. Trophic behaviour and functional morphology of the feeding appendages of the laomediid shrimp Axianassa australis (Crustacea: Decapoda: Thalassinidea). J. Mar. Biol. Assoc. U. K. 81(3):441-454.
CUNHA, I. 2003. Conflito ambiental em águas costeiras: Relação porto-cidade no Canal de São Sebastião. Ambient. soc. 6(2):83-98.

DEN HARTOG, C. 1970. Halodule emarginata nov. sp., a new sea-grass from Brazil (Potamogetonaceae). Blumea 18:65-66

DENADAI, M.R., AMARAL, A.C.Z. \& TURRA, A. 2005. Structure of molluscan assemblages in sheltered intertidal unconsolidated environments. Braz. Arch. Biol. Techn. 48:825-839.

DENADAI, M.R., ARRUDA, E.P., DOMANESCHI, O. \& AMARAL, A.C.Z. 2006. Veneridae (Mollusca, Bivalvia) da costa norte do Estado de São Paulo. Biota Neotrop. 6(3): http://www.biotaneotropica.org.br/v6n3/pt/a bstract?inventory+bn01106032006 (último acesso em 23/10/1009).

DITADI, A.S.F. 1982a. Intertidal sipunculans (Sipunculus) from Southern Brazil. Rev. bras. biol. 42(4):785-800.

DITADI, A.S.F. 1982b. On the burrows of echiuran worms (Echiura): a survey. Bol. Zool. Univ. S. Paulo 7:21-36.

DITADI, A.S.F. 1983a. O filo Echiura. Cienc. cult. 35(4):421-426.

DITADI, A.S.F. 1984. Field and laboratory observations on the echiuran worm, Lissomyema exilii. Uttar Pradesh J. Zool. Muzaffarnagar 4(1):17-21.

DITADI, A.S.F. \& MENDES, E.G. 1986. Respiration studies on an echiuran worm Lissomyema exilii. Bol. Fisiol. Animal 10:41-60.

DITADI, A.S.F. \& MIGOTTO, A.E. 1981. On Golfingia (Nephasoma) confusa (Sluiter, 1902), Sipuncula. Pap. Avul. Zool. 34(10):125-134.

DOMINCIANO, L.C.C., SANT'ANNA, B.S. \& TURRA, A. 2009. Are the preference and selection patterns of hermit crabs for gastropod shells species-or site-specific? J. Exp. Mar. Biol. Ecol. 378:15-21.

DUARTE, L.F.L. \& MORGADO, E.H. 1983. Crustáceos parasitas de invertebrados associados à esponja Zygomycale parishii (Bowerbank) e ao briozoário Schizoporella unicornis (Johnston). Iheringia Ser. Zool. 62:3-11.

DUARTE, L.F.L. \& NALESSO, R. 1996. The sponge Zygomycale parishii (Bowerbank) and its endobiotic fauna. Estuar. Coast. Shelf S. 42(2):139-151.

DWORSCHAK, P.C. \& COELHO, V.R. 1999. On two alpheids from Araçá (São Paulo, Brazil) with a description of a new species of Leptalpheus (Decapoda: Caridea: Alpheidae). Ann. Naturhist. Mus. Wien 101(B):475-488.

DWORSCHAK, P.C. \& RODRIGUES, A. 1997. A modern analogue for the trace fossil Gyrolithes: burrows of the thalassinidean shrimp Axianassa australis. Lethaia 30(1):41-52.

FAUCHALD, K. 1992. A review of the genus Eunice (Polychaeta: Eunicidae) based upon type material. Sm. C. Zool. 523:1-422.

GENOFRE, G.C., LOWENTHAL, C. \& MOSSOLIN, E.C. 2000. Short-term effect of eyestalk ablation on the respiratory metabolism of Callinectes danae, Smith, 1869 (Crustacea, Decapoda, Portunidae). Biol. Health Sci. J. 2(1-3):5-11.

GONÇALVES, R.R., MASUI, D.C., MCNAMARA, J.C., MANTELATTO, F.L.M., GARÇON, D.P., FURRIEL, R.P.M. \& LEONE, F.A. 2006. A kinetic study of gill $(\mathrm{Na}+, \mathrm{K}+)$-ATPase, and its role in ammonia excretion in the intertidal hermit crab Clibanarius vittatus. Comp. Biochem. Phys. A. 145(3):346-356.

GORNI, R. \& WEBER, R.R. 2004. Organochlorine pesticides residues and PCBs in benthic organisms of the inner shelf of the São Sebastião channel, Sao Paulo, Brazil. Braz. J. Oceanogr. 52(2):141-152.

GUBITOSO, S., DULEBA, W., TEODORO, A.C., PRADA, S.M., ROCHA, M.M., LAMPARELLI, C.C., BEVILACQUA, J.E. \& MOURA, D.O. 2008. Estudo geoambiental da região circunjacente ao emissário submarino de esgoto do Araçá, São Sebastião, SP. Rev. Bras. Geocienc. 38(3):467-475

JORGE, F.B. \& DITADI, A.S.F. 1969. Biochemical studies on Lissomyema exilii (F. Müller, 1883) (Echiura). Comp. Biochem. Phys. 28:817-827.

JORGE, F.B., PETERSEN, J.A. \& DITADI, A.S.F. 1969a. Influence of prolonged fasting on the biochemistry of Lissomyema exilii (Echiura). Comp. Biochem. Phys. 31:483-492. 
JORGE, F.B., PETERSEN, J.A. \& DITADI, A.S.F. 1969b. Iodine accumulation by the nephridia of Sipunculus (Sipuncula). Experientia (Basel) 25:1147-1148.

JORGE, F.B., PETERSEN, J.A. \& DITADI, A.S.F. 1970. Comparative biochemical studies in Sipunculus nudus and Sipunculus multisulcatus (Sipuncula). Comp. Biochem. Phys. 35:153-177.

JORGE, F.B., PETERSEN, J.A., DITADI, A.S.F. \& SAWAYA, P. 1966. Biochemical studies in a eunicid polychaete of the littoral of São Paulo, Brazil. Comp. Biochem. Phys. 17:535-551.

KATTAR, M.R. 1970. Estudo dos protozoários ciliados psamófilos do litoral brasileiro. Bol. zool. biol. mar. 27(1): 123-206.

KIHARA, T.C. \& ROCHA, C.E.F. 1993. Two new species of Hemicyclops (Copepoda: Poecilostomatoida: Clausidiidae) associated with mud shrimps of the genus Callichirus from Brazil. Bijdr. Dierkd. 63(4):243-254.

KIHARA, T.C., BJÖRNBERG, T.K.S. \& KAWAUCHI, G.Y. 2007. Myzomolgus sipunculensis sp. n. (Cyclopoida, Catiniidae), a new copepod associated with sipunculan worms from Brazil. Rev. Bras. Zool. 24:591-600.

KIHARA, T.C., ROCHA, E.F. \& SANTOS, C. 2005. A new species of Catinia Bocquet \& Stock, 1957 (Copepoda, Catiniidae) associated with mud shrimps, Axianassa australis Rodrigues \& Shimizu, 1992 (Decapoda, Thalassinidea, Laomediidae), from Brazil. Zootaxa 1039:39-55.

LEITE, F.P.P. 1995. Distribuição temporal e espacial de Kalliapseudes schubarti Mañe-Garzon, 1949 (Tanaidacea, Crustacea) da região do Araçá, São Sebastião, SP. Arq. Biol. Tecnol. 38(2):605-618.

LEITE, F.P.P., TURRA, A. \& GANDOLFI, S.M. 1998. Hermit crabs (Crustacea: Decapoda: Anomura), gastropod shells and environmental stress: their relationship in southeastern Brazil. J. Nat. Hist. 32:1599-1608.

LEITE, F.P.P., TURRA, A. \& SOUZA, E.F.C. 2003. Population biology and distribution of the tanaid Kalliapseudes schubarti (Mañé-Garzon, 1949) in southeastern Brazil. Braz. Journ. Biol. 63: 469-479.

MACHA, N. \& DITADI, A.S.F. 1972. On the epidermial mucous secretion of Lissomyema exilii on echiuran worm. Ann. Histochim. 17:325-332.

MANNING, R.B. \& LEMAITRE, R. 1993. Sergio, a new genus of ghost shrimp from the Americas (Crustacea: Decapoda: Callianassidae). Nauplius 1:39-43.

MARCUS, E. \& MARCUS, E. 1968. Neue brasilianische Loxozomen. Zool. Beitr. 14:203-212.

MARCUS, E. \& MARCUS, E. 1970. Opisthobranchs from Curaçao and faunistically related regions. Stud. Fauna Curacao Caribb. Isl. 33:1-129.

MCNAMARA, J.C. \& MOREIRA, G.S. 1983. Ultrastructure of chromatophores from the fiddler crabs Uca rapax (Smith) and Uca uruguayensis (Nobili) (Decapoda, Brachyura). Crustaceana 44:301-309.

MCNAMARA, J.C., ZANOTTO, F.P. \& ONKEN, H. 2005. Adaptation to hypoosmotic challenge in brachyuran crabs: a microanatomical and electrophysiological characterization of the intestinal epithelia. J. Exp. Zool. 303(A):880-893.

MIGOTTO, A.E. 1996. Benthic shallow-water hydroids (Cnidaria, Hydrozoa) of the coast of São Sebastião, Brazil, including a checklist of Brazilian hydroids. Zool. Verh. 306:1-125.

MIGOTTO, A.E. 1998. The life cycle of Sertularia marginata Kirchenpauer, 1864 (Cnidaria, Hydrozoa): a medusoid-producing sertulariid. J. Nat. Hist. 32:1-12.

Migotto, A.E., TIAGO, C.G. \& MAGAlHÃES, A.R.M. 1993. Malacofauna marinha da região costeira do Canal de São Sebastião, SP, Brasil: Gastropoda, Bivalvia, Polyplacophora e Scaphopoda. Bolm. Inst. oceanogr. S. Paulo 41(1-2):13-27.

MIRANDA, I. \& MANTELATTO, F.L.M. (prelo-a). Estimating population features of the anomuran crab Petrolisthes armatus (Gibbes, 1850) in a remaining and impacted mangrove area of western Atlantic. Biodivers. Conserv.

MIRANDA, I. \& MANTELATTO, F.L.M. (prelo-b). Interaction between the parasite isopod Aporobopyrus curtatus (Crustacea: Isopoda: Bopyridae) and the anomuran crab Petrolisthes armatus (Crustacea: Porcellanidae) in southern Atlantic. Proc. Biol. Soc. Wash.

MOREIRA, G.S. \& NIPPER, M.G. 1978. Association of Stylactis hooperi Sigerfoos (Cnidaria, Hydrozoa) with mollusks and hermit crabs. Cienc. cult., Supl. 30:586.

MOREIRA, G.S., LEITE, L.R. \& NIPPER, M.G. 1978. Notes on Dipurena reesi Vannucci 1956 (Hydrozoa, Corynidae) with a description of an unusual method of asexual reproduction. Bol. Fisiol. Animal Univ. S. Paulo 2:159-164.

MORGADO, E.H. \& AMARAL, A.C.Z. 1981a. Anelídeos poliquetos associados a um briozoário. I. Eunicidae, Lumbrineridae, Lysaretidae e Dorvilleidae. Iheringia Ser. Zool. 60:33-54.

MORGADO, E.H. \& AMARAL, A.C.Z. 1981b. Anelídeos poliquetos associados a um briozoário. II. Palmyridae. Bolm. Inst. oceanogr. S. Paulo 30(1):87-89.

MORGADO, E.H. \& AMARAL, A.C.Z. 1981c. Anelídeos poliquetos associados a um briozoário. III. Polynoidae. Bolm. Inst. oceanogr. S. Paulo 30(1):91-96.

MORGADO, E.H. \& AMARAL, A.C.Z. 1984a. Anelídeos poliquetos associados ao briozoário Schizoporella unicornis (Johnston). IV Phyllodocidae e Hesionidae. Rev. Bras. Zool. 2(2):49-54.

MORGADO, E.H. \& AMARAL, A.C.Z. 1985. Anelídeos poliquetos associados ao briozoário Schizoporella unicornis (Johnston). V. Syllidae. Rev. Bras. Zool. 3(4):219-227.

MORGADO, E.H. \& TANAKA M.O. 2001. The macrofauna associated with the bryozoan Schizoporella errata (Walters) in southeastern Brazil. Sci. Mar. 65(3):173-181.

MUNIZ, P. \& PIRES, A.M.S. 1999. Trophic structure of polychaetes in the São Sebastião Channel (southeastern Brazil). Mar. Biol. 134:517-528.

MUNIZ, P. \& PIRES, A.M.S. 2000. Polychaete associations in a subtropical environment (São Sebastião Channel, Brazil). Mar. Ecol. 21(2):145-160.

MUNIZ, P., VENTURINI, N., PIRES-VANIN, A.M.S., TOMMASI, L.R. \& BORJA, A. 2005. Testing the applicability of a Marine Biotic Index (AMBI) to assessing the ecological quality of soft-bottom benthic communities, in the South America Atlantic region. Mar. Poll. Bull. 50(6):624-637.

NALESSO, R.C., DUARTE, L.F.L., PIEROZZI JUNIOR, I. \& ENUMO, E.F. 1995. Tube epifauna of the Polychaete Phyllochaetopterus socialis Claparède. Est. Coast. Shelf. Sci. 41(1):91-100.

NIPPER-BUSCARIOLLI, M. 1983. Combined effects of temperature and salinity on Stylactis hooperi Sigerfoos 1899 (Hydrozoa, Hydractiniidae). II. Polyp growth and tentacle development. Bol. Fisiol. Animal, Univ. S. Paulo 7:41-48.

NIPPER-BUSCARIOLLI, M. \& MOREIRA, G.S. 1982. Influence of biotic and abiotic factors on the development of Stylactis hooperi (Hydrozoa, Hydractiniidae). Atlantica 5(2):86.

NIPPER-BUSCARIOLLI, M. \& MOREIRA, G.S. 1983. Combined effects of temperature and salinity on Stylactis hooperi Sigerfoos 1899 (Hydrozoa, Hydractiniidae). I. Colony growth, development of medusa buds and hydranth degeneration. Stud. Neotrop. Fauna E. 18:111-120.

NOGUEIRA, J.M.M. 2003. A new species of Paraeupolymnia Young and Kritzler, 1986 (Polychaeta, Terebellidae) from Brazil. Sci. Mar. 67(4):403-411.

NOGUEIRA, J.M.M. \& ALVES, T.M. 2006. Two new terebellid polychaetes (Polychaeta: Terebellidae) from the state of São Paulo, southeastern Brazil. Zootaxa 1205:31-54.

NOGUEIRA, J.M.M. \& FUKUDA, M.V. 2008. A new species of Trypanosyllis Claparède, 1864 (Polychaeta: Syllidae) from Southeastern Brazil, together with a redescription of Brazilian material of Trypanosyllis zebra (Grube, 1860). J. Mar. Biol. Assoc. U. K. 88(5):913-924.

NOGUEIRA, J.M.M., LÓPEZ, E. \& ROSSI, M.C.S. 2004a. Kirkia heterobranchiata, a new genus and species extratubular brooding sabellid (Polychaeta: Sabellidae) from São Paulo, Brazil. J. Mar. Biol. Assoc. U. K. 84:701-710. 
NOGUEIRA, J.M.M., ROSSI, M.C.S. \& LÓPEZ, E. 2006. Intertidal species of Branchiomma Kölliker and Pseudobranchiomma Jones (Polychaeta: Sabellidae: Sabellinae) occurring on rocky shores along the state of São Paulo, southeastern Brazil. Zool. Stud. 45(4):586-610.

NONATO, E.F. 1965. Eunice sebastiani sp. nov. (Annelida, Polychaeta). Bolm. Inst. oceanogr. S. Paulo 14:133-139.

NUCCI, P.R., TURRA, A. \& MORGADO, E.H. 2001 Diversity and distribution of crustaceans from 13 sheltered sandy beaches along São Sebastião Channel, south-eastern Brazil. J. Mar. Biol. Assoc. U. K. 81:475-484.

OMENA, E.P. \& AMARAL, A.C.Z. 1997. Distribuição espacial de Polychaeta (Annelida) em diferentes ambientes entremarés de praias de São Sebastião (SP). Oecol. bras. 3:183-196.

PETERSEN, J.A. \& DITADI, A.S.F. 1971. Asexual reproduction in Glossobalanus crozieri (Ptychoderidae, Enteropneusta, Hemichordata). Mar. Biol. 9:78-85.

PRADO, A. \& MELO, G.A.S. 2002. The genus Planes Bowdich (Decapoda, Grapsidae) along the Brazilian coast. Crustaceana 75(3):579-595.

RANGEL, M., SANCTIS, B., FREITAS, J.C., POLATTO, J.M., GRANATO, A.C., BERLINCK, R.G.S. \& HAJDU, E. 2001. Cytotoxic and neurotoxic activities in extracts of marine sponges (Porifera) from southeastern Brazilian coast. J. Exp. Mar. Biol. Ecol. 262:31-40.

RIBEIRO, S.M., OMENA, E.P. \& MURICY, G. 2003. Macrofauna associated to Mycale microsigmatosa (Porifera, Demospongiae) in Rio de Janeiro state, SE Brazil. Est. Coast. Shelf. Sci. 57:951-959.

RIZZO, A.E., STEINER, T.M. \& AMARAL, A.C.Z. 2007. Glyceridae Grube 1850 (Annelida: Polychaeta) from Southern and Southeastern Brazil, including a new species of Glycera. Biota Neotrop. 7(3): http://www. biotaneotropica.org.br/v7n3/pt/abstract?article+bn00407032007 (ultimo acesso em 23/10/2009).

ROCHA, R.M. \& MONNIOT, F. 1995. Taxonomic and ecological notes on some Didemnum species (Ascidiacea, Didemnidae) for São Sebastião Channel, southeast Brazil. Rev. bras. biol. 55(4):639-649.

RODRIGUES, S.A. 1962. Algumas ascídias do litoral sul do Brasil. Bol. Fac. Filos., Cienc. Let. Univ. Sao Paulo. Zool. 24:193-216.

RODRIGUES, S.A. 1971. Mud shrimps of the genus Callianassa Leach from the Brazilian coast (Crustacea, Decapoda). Arq. zool. 20(3):191-223.

RODRIGUES, S.A. \& MANNING, R.B. 1992. Two new callanassid shrimps from Brazil (Crustacea: Decapoda: Thalassinidea). Proc. Biol. Soc. Wash. 105(2):324-30.

RODRIGUES, S.A. \& ROCHA, R.M. 1993. Littoral compound ascidians (Tunicata) from São Sebastião, Estado de São Paulo, Brazil. Proc. Biol. Soc. Wash. 106(4):728-739.

RODRIGUES, S.A. \& SHIMIZU, R.M. 1992. Description of a new Axianassa (Crustacea: Decapoda: Thalassinidea) from Brazil, and its first larval stage. Proc. Biol. Soc. Wash. 105(2):317-23.

ROSSI-WONGTSCHOWSKI, C.L.D.B., SOARES, L.S.H. \& MUTO, E.Y. 1997. A ictiofauna demersal do Canal e da Plataforma Interna de São Sebastião. Relat. int. Inst. Oceanogr. 41:47-64.

SAKAI, K. 1999. Synopsis of the family Callianassidae. Zool. Verh. 326:1-152

SAWAYA, P. 1950. Reencontro de Balanoglossus gigas Fr. Müller no litoral brasileiro. Bol. Inst. Paul. Oceanogr., 1(1):135-138.

SAWAYA, P. 1951. Balanoglossus gigas Fr. Müller rediscovered on the Brazilian Coast. Nature 167:730-731.

SAWAYA, P. \& LEAHY, W.M. 1971. Fisioecologia e etologia de Aplysia L. (Mollusca - Opistobranchia). Bol. zool. biol. mar. 28:1-17.

SCEMES, E., SALOMÃO, L.C., MCNAMARA, J.C. \& CASSOLA, A.C. 1991. Lack of osmoregulation in Aplysia brasiliana: correlation with response of neuron R15 to osphradial stimulation. Am. J. Physiol. 260:R777-R784

SILVA, L.S., MIRANDA, L.B. \& CASTRO FILHO, B.M.C. 2005. Numerical study of circulation and thermohaline structure in the São Sebastião channel. Rev. bras. geofis. 23:407-425.
STEINER, T.M. \& AMARAL, A.C.Z. 2000. Two new species of Marphysa Quatrefages, 1865 (Eunicidae, Polychaeta) from intertidal sandy beaches of the São Sebastião Channel, State of São Paulo (Brazil). B. Mar. Sci. 67(1):479-489.

STEINER, T.M. \& AMARAL, A.C.Z. 2009. Arabella aracaensis, a new species with growth rings on its mandibles, and some remarks on the endoparasitic Labrorostratus prolificus (Polychaeta: Oenonidae) from southeastern Brazil. J. Nat. Hist. 43(41):2537-2551.

STEINER, T.M. \& SANTOS, C.S.G. 2004. A new species of Neanthes (Annelida, Polychaeta, Nereididae) from Brazil, and some remarks, on Neanthes bruaca Lana \& Sovierzoski, 1987. Beaufortia 54(2):39-57.

TEOdoro, A.C., DUlebA, W., GUBitoso, S., PRADA, S.M., LAMPARELLI, C.C. \& BEVILACQUA, J.E. (prelo). Integration of geochemical and foraminifers assemblage analyses as a tool for environmental characterization of th Araçá and Saco da Capela domestic sewage submarine outfalls, São Sebastião channel, Brazil. Mar. Poll. Bull.

TURRA, A. 2003. Shell condition and adequacy of three sympatric intertidal hermit crab populations. J. Nat. Hist. 37:1781-1796.

TURRA, A. 2004. Intersexuality in hermit crabs: reproductive role and fate of gonopores in intersex individuals. J. Mar. Biol. Assoc. U. K. 84(04):757-759.

TURRA, A. 2005. Reproductive behavior of intertidal hermit crabs in Southeastern Brazil. Rev. Bras. Zool. 22:313-319.

TURRA, A. 2007. Reproductive role of intersex hermit crabs. Notes and news. Crustaceana 80(4):491-494.

TURRA, A. \& DENADAI, M.R. 2001. Desiccation tolerance of four tropical intertidal hermit crabs (Decapoda, Anomura). Mar. Freshw. Behav. Phy. 34(4):227-238.

TURRA, A. \& DENADAI, M.R. 2002. Substrate use and selection in sympatric intertidal hermit crab species. Braz. Journ. Biol. 62(1):107-112.

TURRA, A. \& DENADAI, M.R. 2003. Daily activity of four tropical intertidal hermit crabs from southeastern Brazil. Braz. Journ. Biol. 63(3):537-544

TURRA, A. \& DENADAI, M.R. 2004. Interference and exploitation components in interespecific competition between sympatric intertidal hermit crabs. J. Exp. Mar. Biol. Ecol. 310(2):183-193.

TURRA, A. \& LEITE, F.P.P. 2000. Population biology and growth of three sympatric species of intertidal hermit crabs in south-eastern Brazil. J. Mar. Biol. Assoc. U. K. 80:1061-1069.

TURRA, A. \& LEITE, F.P.P. 2001. Fecundity of three sympatric populations of hermit crabs (Decapoda, Anomura, Diogenidae). Crustaceana 74(10):1019-1027.

TURRA, A. \& LEITE, F.P.P. 2002. Shell utilization patterns of a tropical intertidal hermit crab assemblage. J. Mar. Biol. Assoc. U. K. 82:97-107.

TURRA, A. \& LEITE, F.P.P. 2003. The molding hypothesis: linking shell use with hermit crab growth, morphology, and shell-species selection. Mar. Ecol. Prog. Ser. 265:155-163.

TURRA, A. \& LEITE, F.P.P. 2004. Shell-size selection by intertidal sympatric hermit crabs. Mar. Biol. 145(2):251-257.

TURRA, A. \& LEITE, F.P.P. 2005. Predation on gastropods by shell-breaking crabs: effects on shell availability to hermit crabs. Mar. Ecol. Prog. Ser. 286:279-291.

TURRA, A. \& LEITE, F.P.P. 2007. Embryonic development and duration of incubation period of tropical intertidal hermit crabs (Decapoda, Anomura). Rev. Bras. Zool. 23:677-686.

VERGAMINI, F.G. \& MANTELATTO, F.L.M. 2008a. Continuous reproduction and recruitment in the narrowback mud crab Panopeus americanus (Brachyura, Panopeidae) in a remnant human-impacted mangrove area. Invertebr. reprod. dev. 51:1-10.

VERGAMINI, F.G. \& MANTELATTO, F.L.M. 2008b. Microdistribution of juveniles and adults of the mud crab Panopeus americanus (Brachyura, Panopeidae) in a remnant mangrove area in the southwest Atlantic. J. Nat. Hist. 42(23-24):1581-1589. 
VERNBERG, W.B., MOREIRA, G.S. \& MCNAMARA, J.C. 1981. The effect of temperature on the respiratory metabolism of the developmental stages of Pagurus criniticornis (Dana) (Anomura: Paguridae). Mar. Biol. Lett. 2:1-9.

WEHRTMANN, I.S., MIRANDA, I. \& MANTELATTO, F.L.M. (prelo). A latitudinal comparison of reproductive aspects of Petrolisthes armatus (Anomura, Porcellanidae) between Pacific and Atlantic populations. J. Crust. Biol.

ZINNER, K. \& VANI, Y.S. 1986. Some characteristics of the mucus of the bioluminescent polychaete, Chaetopterus variopedatus. Bol. Fisiol. Animal, Univ. S. Paulo 10:7-14.

\section{Livro e capítulos}

AMARAL, A.C.Z., VOLKMER-RIBEIRO, C., MANSUR, M.C.D., SANTOS, S.B., AVELAR, W.E.P., MATTEWS-CASCON, H., LEITE, F.P.P., MELO, G.A.S., COELHO, P.A., BUCKUP, G.B., BUCKUP, L., VENTURA, C.R.R. \& TIAGO, C.G. 2008. A Situação de Ameaça dos Invertebrados Aquáticos no Brasil. In Livro Vermelho da Fauna Brasileira Ameaçada de Extinção (A.B.M. Machado, G.M. Drummond \& A.P. Paglia, orgs). Ministério do Meio Ambiente - MMA, Brasilia, DF, v. 1, p. 156-351.

FRANCISCO, J. \& CARVALHO, P.F. 2003. Desconstrução do lugar - o aterro da praia da frente do centro histórico de São Sebastião - SP. In Ambientes - estudos de Geografia (L.H. Oliveira, org.). AGETEO, Rio Claro, SP, v. 1, p. 105-119.

LAMPARELLI, C.C., MOURA, D.O., VINCENT, R.C., RODRIGUES, F.O., LOPES, C.F. \& MILANELLI, J.C.C. 1998. Mapeamento dos ecossistemas costeiros do Estado de São Paulo. Secretaria do Meio Ambiente, CETESB, São Paulo.

RODRIGUES, S.A. \& SHIMIZU, R.M. 1998. Thalassinidea. In Catalogue of the Crustacea from Brazil (P.S. Young, ed.). Museu Nacional, Rio de Janeiro, n. 6, p. 379-385. (Série Livros).

SAWAYA, P. 1964. Enteropneustos. In História Natural de Organismos Aquáticos do Brasil (P.E. Vanzolini, ed.). FAPESP, São Paulo, p. 309-316.

\section{Trabalhos completos e resumos expandidos publicados em anais de congressos}

AMARAL, A.C.Z., DENADAI, M.R., ARRUDA, E.P., RIZZO, A.E., PARDO, E.V., TURRA, A., STEINER, T.M., SALVADOR, L.B., OMENA, E.P., NUCCI, P.R., ABRAHÃO, J.R. \& REIS, M.O. 2000. Biodiversidade da macrofauna bêntica entremarés de praias da região subtropical da costa brasileira. In Resumos I Simpósio Brasileiro sobre Praias Arenosas Morfodinâmica, Ecologia, Uso, Riscos e Gestão. Itajaí, SC, p. 260-262.

ARRUDA, E.P., DENADAI, M.R., SALVADOR, L.B. \& AMARAL, A.C.Z. 1997a. Comparação de comunidades de moluscos em diferentes ambientes entremarés de praias do Canal de São Sebastião (SP, Brasil). In Resumos Expandidos VII Congresso Latino-americano sobre Ciências do Mar (COLACMAR). Santos, SP, p. 52-53.

BIAGI, R. \& MANTELATTO, F.L.M. 2005. Efecto de la presencia de los conspecíficos en el proceso de muda y crescimiento en ermitaños (Crustacea, Diogenidae). In Programa y Resumenens del XXV Congreso de Ciencias del Mar de Chile y XI Congreso Latinoamericano de Ciencias del Mar. Viña del Mar, Chile, p. 85-86.

BLUMER, G.J. \& MOREIRA, G.B. 1995. Efeito da temperatura no consumo de oxigênio de Penaeus schmitti Burkenroad, 1936. In Resumos VI Congreso Latinoamericano de Ciencias del Mar Mar del Plata, Argentina, p. 34.

BRASIL-LIMA, G.M.S.S., MOSER, G.A.O. \& AIDAR, E. 1997. Distinction between Araçá Bay and São Sebastião channel waters pointed out by multivariate analysis. In Resumos Expandidos VII Congresso Latino-americano sobre Ciências do Mar (COLACMAR). Santos, SP, p. $110-111$.

DITADI, A.S.F. 1970a. A preliminary note on the mode of life of Lissomyema exilii (F. Müller, 1883), Echiura. In Proceedings of the First International Symposium on the Biology of the Sipuncula and Echiura. Todorovic, Belgrade, Yugoslavia, p. 143-146.
DITADI, A.S.F. 1970b. On the coelomic fluid of Lissomyema exilii (F. Müller, 1883) (Echiura). In Proceedings of the First International Symposium on the Biology of the Sipuncula and Echiura. Todorovic, Belgrade, Yugoslavia, p. 191-196.

DITADI, A.S.F. 1970c. The survival of Lissomyema exilii (F. Müller, 1883) (Echiura) under anaerobic conditions. In Proceedings of the First International Symposium on the Biology of the Sipuncula and Echiura. Todorovic, Belgrade, Yugoslavia, p. 147-150.

FRANCISCO, J. 2000a. A desconstrução total da praia da frente da cidade de São Sebastião (SP). In Resumos I Forum de Debates Ecologia da Paisagem e Planejamento Ambiental - riscos ambientais nos trópicos úmidos: movimentos de massa e inundações. Rio Claro, SP, p. 1-7.

GABRIEL. J.B. \& MOREIRA, G.S. 1995b. Effects of aromatic hydrocarbons in some aspects of the biology of Pagurus criniticornis (Dana) (Crustacea, Paguridae). In Anais do Simpósio Nipo-Brasileiro de Ciências e Tecnologia. ACIESP, n. 96, p. 275-281.

JACOBUCCI, G.B. \& LEITE, F.P.P. 1997. Estrutura dos agrupamentos de tres espécies de ermitões (Decapoda, Anomura) na ilha de Pernambuco, São Sebastião, SP. In Resumos VII Congresso Latino-Americano sobre Ciências do Mar. Santos, SP, p. 26-27.

KAWAKAMI, S.K. \& MONTONE, R.C. 1999. Esteróides em sedimentos de Ubatuba e da Baía do Araçá, São Paulo, como indicadores de contaminação por esgoto doméstico. In Anais VII Congresso Brasileiro de Geoquímica e V Congresso de Geoquímica dos países de língua portuguesa. Porto Seguro, BA, p. 102-104.

MOREIRA, G.S., NIPPER, M.G. \& LEITE, L.R. 1979. On Stylactis hooperi Sigerfoos 1899 (Hydrozoa, Hydractiniidae) a new addition to the fauna of Southern Brazil. In Proceedings International Symposium on Marine Biogeography and Evolution of the Southern Hemisphere. New Zealand, v.2, p. 679-689.

NUCCI, P.R., MORGADO, E.H. \& AMARAL, A.C.Z. 1997. Aspectos da distribuição de Kalliapseudes schubarti Mañe-Garzon, 1949 (Crustacea, Tanaidacea) na região do Araçá, São Sebastião (SP - Brasil). In Resumos VII Congresso Latino-americano sobre Ciências do Mar (COLACMAR). Santos, SP, p. 223-224.

TEOdoro, A.C., DUlebA, W., Gubitoso, S., PRADA, S.M., LAMPARELLI, C.C., BEVILACQUA, J.E., MOURA, D.O. \& PHILLIPOS, L.S. 2008. Integration of geochemical and foraminifers associations analysis as a tool for environmental characterization of the Araçá domestic sewage submarine outfall, São Sebastião Channel, Brazil. In Proceedings of International Conference on Marine Waste Water, Discharges and Coastal Environmental MWWD 2008. Cavtat, Dubrovnik, p. 150-151.

TEODORO, A.C., DULEBA, W., GUBITOSO, S. \& PRADA, S.M. 2007. Influência da disposição oceânica de esgotos na Baía do Araçá, Canal de São Sebastião, SP. In Anais do XI Congresso Brasileiro de Geoquímica. Atibaia, SP.

TOMINAGA, E.N., RUGNO, N.C. \& FLYNN, M.N. 2006. Processos hidrodinâmicos e sedimentares avaliados na região de São Sebastião, SP. In Environmental and Health World Congress. Proceedings Envieronmental and Health World Congress. Santos, SP, p. 671-675.

TURRA, A. \& LEITE, F.P.P. 1997. Estratégias de ocupação de habitat por três espécies simpatricas de Clibanarius (Decapoda, Anomura, Diogenidae) na ilha de Pernambuco, Araçá, São Sebastião (SP). In Resumos VII Congresso Latino-americano sobre Ciências do Mar. Santos, SP, p. 506-507.

TURRA, A., JACOBUCCI, G.B., ARAÚJO, F.M.P. \& LEITE, F.P.P. 2000. Spatial distribution of four sympatric species of hermit crabs (Decapoda, Anomura). In Fourth International Crustacean Congress. (J.C.V. Klein \& F. Schram, eds.) 1998, A. A. Balkema, Amsterdam, Rotterdam, p. 261-273.

VERGAMINI, F.G. \& MANTELATTO, F.L.M. 2007. Distribuição espacial do caranguejo Panopeus americanus (Crustacea, Brachyura, Panopeidae) na região do Araçá, litoral norte de São Paulo, Brasil. In Resumos do XII Congresso Latino-Americano de Ciências do Mar. Florianópolis, SC, p. 27-30. 


\section{Resumos publicados em anais de congressos}

AGUJARO, L.F. \& LEITE, F.P.P. 1991. Microflora bêntica da região entremarés do Araçá, São Sebastião, Litoral Norte do Estado de São Paulo. In II Simpósio sobre Oceanografia. São Paulo, SP, p. 255.

AMARAL, A.C.Z. 1996. Monitoramento ambiental como subsidio para manejo em praias arenosas. In III Reunião Especial da SBPC Ecossistemas Costeiros. Florianópolis, SC, p. 127-128.

AMARAL, A.C.Z. \& MORGADO, E.H. 1987. Padrões de distribuição de anelídeos poliquetas na Praia do Araçá, São Sebastião, SP. In VI MiniSimpósio de Biologia Marinha. São Sebastião, SP, p. 5-6.

AMARAL, A.C.Z. \& MORGADO, E.H. 1989. Avaliação da composição e densidade dos anelídeos poliquetos da região entremarés do Araçá (São Sebastião, SP). In I Simpósio sobre Oceanografia. São Paulo, SP, p. 96-97.

AMARAL, A.C.Z. \& MORGADO, E.H. 1991. Alteraciones en la fauna de anélidos poliquetos de Araçá, São Sebastião (SP, Brasil). In Congresso Latino Americano de Administracion de la Zona Costera. Ensenada, México, p.1.

AMARAL, A.C.Z., MORGADO, E.H., FERREIRA, C.P. \& LEITE, F.P.P. 1988. Distribuição da macrofauna bêntica da zona entremarés, em praias do litoral do Estado de São Paulo. In VII Mini-Simpósio de Biologia Marinha. São Sebastião, SP, p. 8.

AMARAL, A.C.Z., MORGADO, E.H., FERREIRA, C.P. \& LEITE, F.P.P. 1989. Macrofauna bêntica da zona entremarés em praias do litoral do Estado de São Paulo. In XVI Congresso Brasileiro de Zoologia. João Pessoa, PB, p. 233.

AMARAL, A.C.Z., MORGADO, E.H., FERREIRA, C.P., LEITE, F.P.P., BELUCIO, L.F. \& LOPES, P.P. 1989. Distribuição da macrofauna bêntica da zona entremarés em praias do litoral do Estado de São Paulo. In VIII Mini-Simpósio de Biologia Marinha. São Sebastião, SP, p. 7.

AMARAL, A.C.Z., MORGADO, E.H., FERREIRA, C.P., HENRIQUES, S.A., STEINER, T.M., OMENA, E.P., RIZZO, A.E., ABRAHÃO, J.R., NUCCI, P.R., PARDO, E.V., SALVADOR, L.B., LIMA, L.H. \& REIS, M.O. 1995. Monitoramento de praias arenosas do Canal de São Sebastião. In X Mini-Simpósio de Biologia Marinha. São Sebastião, SP, p. 3.

AMARAL, A.C.Z., MORGADO, E.H. \& STEINER, T.M. 1994. Alguns aspectos da zonação da macrofauna de poliquetos em praias arenosas. In IX Mini-Simpósio de Biologia Marinha. São Sebastião, SP, p. 2.

AMARAL, A.C.Z., MORGADO, E.H., STEINER, T.M. \& SALVADOR, L.B. 1995. Importance of substrate on the distribution of sandy beaches macroinfauna. In Land-Ocean Interactions in the Coastal Zone (LOICZ). São Paulo, SP, p. 65.

ARANTES, I.C. \& LEITE, F.P.P. 1989. Distribuição e aspectos biológicos dos ermitões da região do Araçá, São Sebastião, SP. In Resumos VIII Mini-Simpósio de Biologia Marinha. São Sebastião, SP, p. 8.

ARANTES, I.C. \& LEITE, F.P.P. 1991a. Composição e distribuição das espécies de Clibanarius (Crustacea, Anomura) da região do Araçá, São Sebastião, SP. In XVIII Congresso Brasileiro de Zoologia. Salvador, BA, p. 532.

ARANTES, I.C. \& LEITE, F.P.P. 1991b. Os ermitões do Araçá, São Sebastião, SP. Composição, distribuição e utilização das conchas. In II Simpósio sobre Oceanografia. São Paulo, SP, p. 171.

ARANTES, I.C. \& LEITE, F.P.P. 1992. Dieta alimentar de duas espécies de ermitões da região do Araçá, São Sebastião, SP. In XIX Congresso Brasileiro de Zoologia e XII Congresso Latino-Americano de Zoologia, Belém, PA, p. 31.

ARANTES, I.C. \& LEITE, F.P.P. 1994. Seleção de conchas por ermitões do Araçá, São Sebastião, SP. In Resumos IX Mini-Simpósio de Biologia Marinha. São Sebastião, SP, p. 2-3.

ARANTES, I.C. \& LEITE, F.P.P. 1995. Utilização e seleção de conchas por ermitões da zona entremarés na região do Araçá, São Sebastião, SP, Brasil. In VI Congresso Latinoamericano de Ciencias del Mar. Mar Del Plata, Argentina, p. 22.
ARASAKI, E. \& PIRES-VANIN, A.M.S. 1997. Distribuição e estrutura da macrofauna bêntica no Canal de São Sebastião, São Paulo, Brasil. In XIl Simpósio de Biologia Marinha. São Sebastião, SP, p. 10.

ARAÚJO, F.M.P. \& LEITE, F.P.P. 1996. Estrutura populacional, distribuição e reprodução do ermitão Pagurus criniticornis Dana, 1852 (Decapoda, Anomura, Paguridae) na região do Araçá, São Sebastião, SP. In XI Simpósio de Biologia Marinha. São Sebastião, SP, p. 13.

ARRUDA, E.P. \& AMARAL, A.C.Z. 1998. Composição e diversidade específica das comunidades de moluscos entremarés de praias do Canal de São Sebastião, SP. In XIII Simpósio de Biologia Marinha. São Sebastião, SP, p. 15.

ARRUDA, E.P. \& AMARAL, A.C.Z. 1999. Variação temporal dos moluscos na região entremarés de praias do Canal de São Sebastião. In XIV Simpósio de Biologia Marinha. São Sebastião, SP, p. 30.

ARRUDA, E.P., DENADAI, M.R., SALVADOR, L.B. \& AMARAL, A.C.Z. 1997b. Comparação de comunidades de moluscos em diferentes ambientes entremarés de praias do Canal de São Sebastião (SP, Brasil). In XII Simpósio de Biologia Marinha. São Sebastião, SP, p. 11.

AVELLAR, L.M., NOGUEIRA, J.M.M. \& SANTOS, C.S.G. 2006. Biodiversidade de poliquetas nereidí́deos (Polychaeta: Nereididae) em costões rochosos do Estado de São Paulo. In $1^{\circ}$ Simpósio LatinoAmericano de Polychaeta. São Sebastião, SP, p. 32.

BARATA, G.F., LEITE, F.P.P. \& LUCA, I.M.S. 1996. Tipos celulares e aspectos da maturação dos ovários de 3 espécies de Clibanarius (Decapoda, Anomura, Diogenidae) na região do Araçá, São Sebastião, SP. In XI Simpósio de Biologia Marinha. São Sebastião, SP, p. 15.

BARRIOS, O.H.A. \& FREITAS, J.C. 1987. Ação do extrato acetônico da glândula hipobranquial de Concholepas concholepas no comportamento alimentar de Pagurus criniticornis no labirinto em "Y". In Resumos VI Mini-Simpósio de Biologia Marinha. São Sebastião, SP, p. 6

BELÚCIO, L.F. 1991. Malacofauna associada a um banco de Anomalocardia brasiliana (Gemelin, 1791) (Mollusca, Veneridae) na região do Araçá, São Paulo, SP. In XII Encontro Brasileiro de Malacologia. São Paulo, SP, p. 26

BELÚCIO, L.F. 1992. Recolonização da zona entremarés na região do Araçá, São Sebastião, SP, por moluscos, estágios iniciais. In XIX Congresso Brasileiro de Zoologia e XII Congresso Latino-Americano de Zoologia. Belém, PA, p. 21.

BELÚCIO, L.F. \& MORGADO, E.H. 1991. Malacofauna associada a um banco de Anomalocardia brasiliana (Gemelin, 1791) (Mollusca, Veneridae) na região do Araçá, São Paulo, SP. In XII Encontro Brasileiro de Malacologia. São Paulo, SP, p. 26.

BELÚCIO, L.F. \& MORGADO, E.H. 1994. Malacofauna bêntica da zona entremarés em praias da região do Araçá, litoral do Estado de São Paulo. In II Congresso de Ecologia do Brasil. Londrina, PR, v.2, p. 362.

BELÚCIO, L.F. \& MORGADO, E.H. 1995. Padrões de distribuição e abundância de moluscos na região entremarés do Araçá (São Sebastião, SP). In X Mini-Simpósio de Biologia Marinha. São Sebastião, SP, p. 4-5.

BIAGI, R. \& MANTELATTO, F.L.M. 2006. Efeito da presença dos conspecíficos no processo de muda e crescimento em ermitões (Diogenidae). In IV Congresso Brasileiro sobre Crustáceos. Guarapari, ES, p. 70.

BJÖRNBERG, T.K.S. \& SANTOS, C. 2002. Novo gênero e espécie de copépodo Laofontídeo (Crustacea, Harpacticoida) parasita dos Xanthidae (Crustacea, Decapoda). In XVII Simpósio de Biologia Marinha. São Sebastião, SP, p. 94.

BRASIL-LIMA, G.M.S.S. 1996. Emissário submarino de São Sebastião como fator eutrofizante para as águas da baia do Araçá. In III Simpósio sobre Oceanografia. São Paulo, p.

CAPRARA, L. \& FREITAS, J.C. 1996. Efeitos da fração hidrossolúvel do petróleo em quimiorreceptores da antênula do siri azul Callinectes danae. In XI Simpósio de Biologia Marinha. São Sebastião, SP, p. 23.

CARVALHO, M.S. \& HAJDU, E.M. 1999. Gênero Halichondria Fleming, 1828 (Halichondrida, Demospongiae) - Quarta nova espécie no Canal 
de São Sebastião e cercanias. In XIV Simpósio de Biologia Marinha. São Sebastião, SP, p. 33.

CASTELO-BRANCO, P., FONSECA, M.G., MALAVASI, G.H., ROCCA, M.A. \& LEITE, F.P.P. 1996. Ocupação de microambientes e escolha de conchas por ermitões (Decapoda, Anomura) em uma praia de São Sebastião, SP. In XI Simpósio de Biologia Marinha. São Sebastião, SP, p. 24.

DITADI, A.S.F. 1967. Ocorrência de Lissomyema mellita (Echiura) no litoral de São Paulo. In Ciência e Cultura, XIX Reunião Anual da Sociedade Brasileira para o Progresso da Ciência. São Paulo, SP, p. 440-441.

DITADI, A.S.F. 1969b. Sobre o metabolismo respiratório de Lissomyema exilii. In IX Congresso Latinoamericano de Ciências Fisiológicas. p. 59.

DITADI, A.S.F. 1983b. Sobre a sistemática e a distribuição de Ochetostoma erythrogrammon (Echiura). In X Congresso Brasileiro de Zoologia. Belo Horizonte, MG, p.345.

DUARTE, L.F.L. 1980a. Ophiactis savignyi (Mull.; Trosch.) (Ophiuroidea) endobionte da esponja Zygomycale parishii (Bowerbank). In Ciência e Cultura, XXXII Reunião Anual da Sociedade Brasileira para o Progresso da Ciência. Rio de Janeiro, RJ, p. 564-565.

DUARTE, L.F.L. 1982. A endofauna da esponja Zygomycale parishii (Bowerbank). In Ciência e Cultura, XXXIV Reunião Anual da Sociedade Brasileira para o Progresso da Ciência. Campinas, SP, p. 611.

FANTUCCI, M.Z. \& MANTELATTO, F.L.M. 2007. Spermatophore morphology of the hermit crab Pagurus criniticornis (Stimpson, 1859) (Anomura, Paguridae) from southern Atlantic, Brazil. In Program and Abstracts of the Crustacean Society Mid-Year Meeting. Coquimbo, Chile, p. 91.

FANTUCCI, M.Z. \& MANTELATTO, F.L.M. 2008. Sistema reprodutor masculino de Pagurus criniticornis (Crustacea, Anomura, Paguridae). In XXVII Congresso Brasileiro de Zoologia. Curitiba, PR, p. 66.

FUKUDA, M.V. \& NOGUEIRA, J.M.M. 2004. Odontosyllis Claparède, 1863 (Polychaeta: Syllidae: Eusyllinae) na zona entremarés de costões rochosos de São Paulo. In XXV Congresso Brasileiro de Zoologia. Brasília, DF, p. 8.

FURTADO, V.V., CEGO, M.C. \& WEBER, R.R. 1986. Modelo de dispersão de óleo na região do Canal de São Sebastião. In V mini-Simpósio de Biologia Marinha. São Sebastião, SP, p. 7.

GABRIEL, J.B. \& MOREIRA, G.S. 1995a. Efeito da temperatura no consumo de oxigênio de Penaeus schmitti (Burkenroad 1936). In X Mini-Simpósio de Biologia Marinha. São Sebastião, SP, p. 12.

GAETA, S.A., SUSINI, S.M., LOPES, R.M., MORAES, M.C.P., METZLER, P.M., ABE, D.S. \& SALDANHA, F.M.P. 1987. Plâncton e produtividade primária no Canal de São Sebastião - Avaliação preliminar durante o inverno de 1987. In VI Mini-Simpósio de Biologia Marinha. São Sebastião, SP, p. 11-12.

GUBITOSO, S., DULEBA, W., TEODORO, A.C., LAMPARELLI, C.C., BEVILACQUA, J.E. \& MOURA, D.O. 2007. Estudo geoambiental da região circunjacente ao emissário submarino de esgoto do Araçá, São Sebastião, SP. In $30^{\text {a }}$ Reunião Anual da Sociedade Brasileira de Química. Águas de Lindóia, SP, p. A30.

GUBITOSO, S., DULEBA, W., TEODORO, A.C. \& PRADA, S.M. 2006. Estudo composicional e textural dos sedimentos da região circunjacente ao emissário submarino de esgoto do Araçá, São Sebastião, SP. In XLIII Congresso Brasileiro de Geologia. Aracaju, SE, p. 67.

GUBITOSO, S., TEODORO, A.C., DULEBA, W. \& PRADA, S.M. 2008. Especiação de fósforo em sedimentos da área próxima aos difusores dos emissários submarinos de efluentes do Araçá e TEBAR, São Sebastião, SP. In $1^{\circ}$ Encontro Brasileiro Sobre Especiação Química. Águas de São Pedro, SP, p. P-67.

IGUCHI, J., VERGAMINI, F.G., TEROSSI, M. \& MANTELATTO, F.L. 2008. Investimento reprodutivo do caranguejo Panopeus americanus (Brachyura, Panopeidae) em uma área de manguezal impactada, São Sebastião, SP. In $16^{\circ}$ Simpósio Internacional de Iniciação Científica da USP (SIICUSP). Ribeirão Preto, SP.
JACOBUCCI, G.B. \& LEITE, F.P.P. 1996. Estrutura dos agrupamentos de ermitões (Decapoda-Anomura) na região do Araçá, São Sebastião-SP. In XI Mini-Simpósio de Biologia Marinha. São Sebastião, SP, p. 40.

JACOBUCCI, G.B. \& LEITE, F.P.P. 1998a. Padrões de movimento de Clibanarius vittatus (Anomura, Diogenidae) na região do Araçá, São Sebastião, São Paulo. In XXII Congresso Brasileiro de Zoologia. Recife, PE, p. 78

JACOBUCCI, G.B. \& LEITE, F.P.P. 1998b. Ocupação de agrupamentos de ermitões (Decapoda, Anomura) na região do Araçá, São Sebastião, São Paulo. In XXII Congresso Brasileiro de Zoologia. Recife, PE.

KAWAUCHI, G.Y. \& MIGOTTO, A.E. 2002. Sipúnculos do projeto "Biodiversidade bêntica marinha no Estado de São Paulo" (BIOTA/ FAPESP). In XVII Simpósio de Biologia Marinha. São Sebastião, SP, p. 119.

LEITE, F.P.P. 1989a. Alterações na distribuição e densidade de Kalliapseudes schubarti Mané-Garzon, 1949, (Crustacea - Tanaidacea) do Araçá, São Sebastião, SP. In I Simpósio sobre Oceanografia. São Paulo, SP, p.190.

LEITE, F.P.P. 1989b. Distribuição da macrofauna da zona entremarés, em praias do litoral do Estado de São Paulo. Alterações da fauna de crustáceos na região do Araçá, São Sebastião, SP. In VIII Mini-Simpósio de Biologia Marinha. São Sebastião, SP, p. 17.

LEITE, F.P.P. 1991a. Distribuição e variação sazonal de Kalliapseudes schubartii Mane-Garzon, 1949 (Crustacea, Tanaidacea) na região do Araçá, São Sebastião, SP. In II Simpósio sobre Oceanografia. São Paulo, SP, p. 172.

LEITE, F.P.P. 1991b. Modificaciones en la composición de la fauna de crustáceos de Araçá, São Sebastião (São Paulo - Brasil). In I Congresso Latino-Americano de Administración de Zona Costera. Ensenada, México, p. 10.

LEITE, F.P.P. \& FERREIRA, C.P. 1988. Composição, distribuição e densidade dos crustáceos do Araçá, São Sebastião, SP. In VII Mini-Simpósio de Biologia Marinha. São Sebastião, SP, p. 27.

LEITE, F.P.P. \& JACOBUCCI, G.B. 1998. Padrões de movimento de Clibanarius vittatus (Anomura, Diogenidae) na região do Araçá, São Sebastião, SP. In XXII Congresso Brasileiro de Zoologia. Recife, PE, p. 78.

LEITE, F.P.P., LEITE, P.E.P. \& TURRA, A. 1995. Desenvolvimento dos ovócitos de três espécies de ermitões da região do Araçá, São Sebastião, SP. In X Mini-Simpósio de Biologia Marinha. São Sebastião, SP, p. 15-16.

LEITE, F.P.P., RAMOS, M.P. \& SOTO-ESPINOZA, D. 1992. Aspectos da dinâmica populacional de Kalliapseudes schubarti Mané-Garzon, 1949 (Crustacea, Tanaidacea) do Araçá, São Sebastião, SP. In XIX Congresso Brasileiro de Zoologia e XII Congresso Latino-Americano de Zoologia. Belém, PA, p. 40.

LEITE, F.P.P, TURRA, A. \& GANDOLFI, S.M. 1996. Relação entre ermitões, estrutura ambiental e disponibilidade de conchas nas praias Grande, Barequeçaba, Preta, Cabelo Gordo e Araçá, São Sebastião, SP. In XI Simpósio de Biologia Marinha. São Sebastião, SP, p. 43.

LEITE, I.L.A. \& NOGUEIRA, J.M.M. 2006. Quatro novas espécies de poliquetas silíneos (Polychaeta: Syllidae: Syllinae). In $1^{\circ}$ Simpósio Latino-Americano de Polychaeta. São Sebastião, SP, p. 35.

LOWENTHAL, C., MOSSOLIN, E.C., GENOFRE, G.C. \& SANTOS, E.A. 1996. Efeito, a curto prazo, da ablação dos pedúnculos oculares no metabolismo respiratório de Callinectes danae Smith, 1869 (Crustacea, Decapoda, Portunidae). In III Encontro de Especialistas em Decapoda Brachyura. Rio Grande, RS, p. 41.

MAGALHÃES, C.A. 1987a. Variação morfológica em Thais haemastoma L. 1767: padrões e fatores ecológicos determinantes. In X Encontro Brasileiro de Malacologia. São Paulo, SP, p. 37.

MARQUES, S.M. \& SAWAYA, M.I. 1972. Comportamento dos crustáceos Uca rapax do mangue de São Sebastião - SP. In XXIV Reunião Anual da Sociedade Brasileira para o Progresso da Ciência, Ciência e Cultura. São Paulo, 24(Suppl. 6):368.

MIRANDA, I.S. \& MANTELATTO, F.L.M. 2006a. Aspectos reprodutivos de Petrolisthes armatus (Crustacea, Porcellanidae) em São Sebastião, SP. In XXVI Congresso Brasileiro de Zoologia. Londrina, PR, p. 8. 
MIRANDA, I.S. \& MANTELATTO, F.L.M. 2006b. Dinâmica populacional de Petrolisthes armatus (Gibbes, 1850) (Anomura, Porcellanidae) em São Sebastião, litoral norte de São Paulo. In IV Congresso Brasileiro sobre Crustáceos. Ribeirão Preto, SP, p. 105.

MIRANDA, I.S. \& MANTELATTO, F.L.M. 2008. Crescimento relativo e maturidade sexual de Petrolisthes armatus (Crustacea, Porcellanidae) na região do Araçá, São Sebastião, SP. In III Congresso Brasileiro de Oceanografia e Congresso Ibero-Americano de Oceanografia. Fortaleza, CE.

MONTOUCHET, P.C.G. 1988. A fauna de moluscos em sedimentos do Araçá na zona das marés (São Sebastião, SP). In VII Mini-Simpósio de Biologia Marinha. São Sebastião, SP, p. 29.

MOREIRA, P.S. 1972a. Sobre Kalliapseudes schubarti Mañe-Garzon, 1949, na Praia do Araçá, São Sebastião, SP. In V Congresso Brasileiro de Zoologia. São Paulo, SP, p. 52.

MOREIRA, P.S. 1972b. Sobre Kalliapseudes schubartii Mané Garzon, 1949, na Praia do Araçá, São Sebastião, SP. In XXIV Reunião Anual da Sociedade Brasileira para o Progresso da Ciência, Ciência e Cultura. São Paulo, 24(Suppl. 6):366.

MORGADO, E.H. 1980a. Fauna associada ao briozoário Schizoporella unicornis (Jonhston,1847). In II Seminário Regional de Ecologia. São Carlos, SP, p. 55.

MORGADO, E.H. 1984. Crustáceos (Decapoda - Reptantia) associados a colônias de Schizoporella unicornis (Bryozoa). In XXXVI Reunião Anual da Sociedade Brasileira para o Progresso da Ciència, Ciência e Cultura. São Paulo, 36(Suppl. 7):913.

MORGADO, E.H. 1985. Ocorrência de moluscos em colônias do briozoário Schizoporella unicornis (Johsnton) no litoral norte do Estado de São Paulo. In XII Congresso Brasileiro de Zoologia. Campinas, SP, p. 40.

MORGADO, E.H. \& AMARAL, A.C.Z. 1981d. Poliquetos de escamas associados a colônias de Schizoporella unicornis (Bryozoa). In III Encontro de Zoologia do Nordeste. Recife, PE, p. 228.

MORGADO, E.H. \& AMARAL, A.C.Z. 1982. Anelídeos poliquetos associados ao briozoário Schizoporella unicornis (Johnston, 1847). In IX Congresso Brasileiro de Zoologia. Porto Alegre, RS, p. 47.

MORGADO, E.H. \& AMARAL, A.C.Z. 1983b. Ocorrência de Syllidae (Annelida, Polychaeta) em colônias de Schizoporella unicornis (Bryozoa). In X Congresso Brasileiro de Zoologia. Belo Horizonte, MG, p. 38.

MORGADO, E.H. \& AMARAL, A.C.Z. 1984. Anelídeos poliquetos sedentários associados ao briozoário Schizoporella unicornis (Jonhston). In XI Congresso Brasileiro de Zoologia. Belém, PA, p. 431.

MORGADO, E.H. \& AMARAL, A.C.Z. 1988. Distribuição dos anelídeos poliquetos na região do Araçá, São Sebastião, SP. In VII Mini-Simpósio de Biologia Marinha. São Sebastião, SP, p. 30.

MORGADO, E.H. \& AMARAL, A.C.Z. 1996. Estrutura trófica de poliquetos da região entremarés. In XXI Congresso Brasileiro de Zoologia. Porto Alegre, RS, p. 21.

MORGADO, E.H. \& DUARTE, L.F.L. 1979. Crustáceos parasitas de invertebrados associados à esponja Zygomycale parishii (Bowerbank) Topsent e ao briozoário Schizoporella unicornis (Jonhston). In IV Congresso da Sociedade Brasileira de Parasitologia. Campinas, SP, p. 117.

MORGADO, E.H., AMARAL, A.C.Z. \& FERREIRA, C.P. 1989. Distribuição da macrofauna bêntica da zona entremarés em praias do litoral do Estado de São Paulo - Alterações da fauna de anelídeos poliquetos do Araçá, São Sebastião, SP. In VIII Mini-Simpósio de Biologia Marinha. São Sebastião, SP, p. 23.

NOGUEIRA, J.M.M. \& FUKUDA, M.V. 2007. A new species of Trypanosyllis Claparède, 1864 (Polychaeta: Syllidae) from Southeastern Brazil, together with a redescription of Brazilian material of Trypanosyllis zebra (Grube, 1860). In 9th International Polychaete Conference. Portland, Maine, USA, p. 162.

NOGUEIRA, J.M.M. \& ROSSI, M.C.S. 2004. Um novo gênero de poliqueta sabelídeo (Polychaeta: Sabellidae) encubador de embriões. In XXV Congresso Brasileiro de Zoologia. Brasília, DF, p. 6-7.
NOGUEIRA, J.M.M., ROSSI, M.C.S., ABBUD, A., FUKUDA, M.V. \& LEITE, I.L.A. 2003. Biodiversidade de poliquetas (Annelida: Polychaeta) em costões rochosos ao longo do Estado de São Paulo. In XVIII Simpósio de Biologia Marinha. p. 37-38.

NUCCI, P.R., MORGADO, E.H. \& AMARAL, A.C.Z. 1997. Aspectos da distribuição de Kalliapseudes schubarti Mañe-Garzon, 1949 (Crustacea, Tanaidacea) na região do Araçá, São Sebastião (SP - Brasil). In XII Simpósio de Biologia Marinha. São Sebastião, SP, p. 33.

OISHI, C.L. 1994. Ocupação de conchas por Pagurus criniticornis (Decapoda: Anomura) na praia do Araçá, São Sebastião-SP. In II Congresso de Ecologia do Brasil. Londrina, PR, p. 361.

OISHI, C.L. \& LEITE, F.P.P. 1994. Ocupação de conchas por Pagurus criniticornis (Decapoda, Anomura) na praia do Araçá, São Sebastião, SP. In II Congresso de Ecologia do Brasil. Londrina, p. 361.

OMENA, E.P., AMARAL, A.C.Z. \& LIMA, L.H. 1994. Efeito da predação sobre a endofauna da zona entremarés da região do Araçá, São Sebastião, SP. In IX Mini-Simpósio de Biologia Marinha. São Sebastião, SP, p. 24.

OMENA, E.P., AMARAL, A.C.Z., MORGADO, E.H., STEINER, T.M., HENRIQUES, S.A., LIMA, L.H. \& ABRAHÃO, J.R. 1996. Composição e distribuição de anelídeos poliquetos em praias do Canal de São Sebastião - Resultados Preliminares. In XXI Congresso Brasileiro de Zoologia. Porto Alegre, RS, p. 21.

POLATTO, J.M., OLIVEIRA, J.S. \& FREITAS, J.C. 2002. Atividade hemolítica e antimitótica da secreção púrpura de Aplysia brasiliana (Mollusca, Opisthobranchia): resultados preliminares. In XVII Simpósio de Biologia Marinha. São Sebastião, SP, p. 35.

PRADA, S.M., GHEDINI, T.M., TEODORO, A.C., DULEBA, W. \& OLIVEIRA, E. 2008. Estudo toxicológico dos sedimentos da Baía do Araçá, São Sebastião - SP, por meio da razão MSE/SVA e foraminíferos como bioindicadores. In $31^{\text {a }}$ Reunião Anual da Sociedade Brasileira de Química. Águas de Lindóia, SP.

REIS, M.O. \& AMARAL, A.C.Z. 1997. Estrutura da comunidade de poliquetas da zona entremarés na região do Araçá, São Sebastião, SP. In XII Simpósio de Biologia Marinha. São Sebastião, SP, p. 38.

REIS, M.O. \& AMARAL, A.C.Z. 1998. Comparative study of polychaete composition from Araçá intertidal region, São Sebastião (SP, Brazil). In Resumos 6th International Polychaete Conference. Curitiba, PR, p. 120.

REIS, M.O., AMARAL, A.C.Z. \& MORGADO, E.H. 1996. Composição da fauna de poliquetos da zona entremarés, na região do Araçá, São Sebastião, SP. In Resumos XI Simpósio de Biologia Marinha. São Sebastião, SP, p. 56.

RODRIGUES, S.A. 2002. CEBIMar 40 anos de USP: um depoimento pessoal. In Resumos XVII Simpósio de Biologia Marinha. São Sebastião, SP,.

RODRIGUES, S.A. \& SHIMIZU, R.M. 1987. Ocorrência do gênero Axianassa Schmitt, 1924 no litoral brasileiro (Crustacea, Decapoda, Thalassinidea). In XIV Congresso Brasileiro de Zoologia. Juiz de Fora, MG, p. 36.

RODRIGUES, S.A., DWORSCHAK, P.C., COELHO, V.R. \& PEZZUTO, P.R. 1995 Burrows of Axianassa australis (Decapoda: Thalassinidea: Laomediidae) from praia do Araçá, SP, Brazil. In The Crustacean Society 1995 Summer Meeting. Fort Pierce, Florida, p. 26-27.

ROSSI, M.C.S. \& NOGUEIRA, J.M.M. 2004. O gênero Branchiomma Kölliker, 1858 (Polychaeta: Sabellidae) em costões rochosos do Estado de São Paulo. In XXV Congresso Brasileiro de Zoologia. Brasília, DF, p. 7.

ROSSI, M.C.S. \& NOGUEIRA, J.M.M. 2007. Four new species of sabellid polychaetes (Polychaeta: Sabellidae) from rocky shores off the State of São Paulo, southeastern Brazil. In 9th International Polychaete Conference. Portland, Maine, USA, p. 108.

SANTOS, C. \& BUENO, S.L.S. 2001. Levantamento de ectosimbiontes de crustáceos decápodes braquiúros e anomuros de São Sebastião. In XVI Simpósio de Biologia Marinha. São Sebastião, SP, p. 59.

SAWAYA, P. \& FREITAS, J.C. 1972. Observações sobre Chaetopterus variopedatus Rénier, 1804 (Polychaeta) do litoral de S. Paulo. In V Congresso Brasileiro de Zoologia. São Paulo, SP, p. 56. 
SAWAYA, P. \& GENOFRE, G.C. 1972. Sobre as cromatoforotropinas de Uca sp. (Crustáceo Decápode). In Resumos V Congresso Brasileiro de Zoologia. São Paulo, SP, p. 54.

SAWAYA, P. \& MARQUES, S.M. 1972. Comportamento dos crustáceos Uca rapax do mangue de São Sebastião - SP. In V Congresso Brasileiro de Zoologia. São Paulo, SP, p. 54.

SILVA, I.M. \& MANTELATTO, F.L. 2006. Dinâmica populacional de Petrolisthes armatus (Gibbes, 1850) (Anomura, Porcellanidae) em São Sebastião, litoral norte de São Paulo. In IV Congresso Brasileiro sobre Crustáceos. Guarapari, ES, p. 105.

SILVA, I.M. \& MANTELATTO, F.L. 2008a. Crescimento relativo e maturidade sexual de Petrolisthes armatus (Crustacea, Porcellanidae) na região do Araçá, São Sebastião. In III Congresso Brasileiro de Oceanografia/ Congresso Ibero-Americano de Oceanografia. Fortaleza, CE.

SILVA, I.M. \& MANTELATTO, F.L. 2008b. Seria a população de Petrolisthes armatus (Porcellanidae) afetada negativamente pela presença de Aporobopyrys curtatus (Bopyridae)? In V Congresso Brasileiro sobre Crustáceos. Gramado, RS, p. 85.

SOUZA, E.C.F. \& AMARAL, A.C.Z. 1998. Ecologia populacional de Kalliapseudes schubarti Mañe-Garzon, 1949 (Crustacea, Tanaidacea) da região do Araçá, São Sebastião, SP. In III Congresso aberto aos estudantes de Biologia. Campinas, SP.

SOUZA, E.C.F., AMARAL, A.C.Z. \& LEITE, F.P.P. 1998. Ecologia populacional de Kalliapseudes schubarti (Mañe-Garzon, 1949) da região do Araçá, São Sebastião, SP. In XXII Congresso Brasileiro de Zoologia. Recife, PE, p. 97.

SOUZA, E.C.F. \& LEITE, F.P.P. 2002. Atração de Pagurus criniticornis (Dana 1852) (Decapoda, Anomura) por eventos de predação simulada. In XVII Simpósio de Biologia Marinha. São Sebastião, SP, p. 36.

SOUZA, E.C.F., TURRA, A. \& LEITE, F.P.P. 2001. Seleção de conchas por Pagurus criniticornis (Dana 1852) (Decapoda, Anomura). In XVI Simpósio de Biologia Marinha. São Sebastião, SP, p. 65.

STEINER, T.M. \& AMARAL, A.C.Z. 1998a. Nereididae, Goniadidae, Glyceridae, Onuphidae and Eunicidae (Annelida, Polychaeta) from intertidal sandy beaches of the São Sebastião Channel, São Paulo (Brazil). In 6th International Polychaete Conference. Curitiba, PR, p. 134.

STEINER, T.M. \& AMARAL, A.C.Z. 1998b. Nereididae, Goniadidae, Glyceridae, Onuphidae and Eunicidae (Annelida, Polychaeta) from intertidal sandy beaches of the São Sebastião Channel, São Paulo (Brazil). In XIII Simpósio de Biologia Marinha. São Sebastião, SP, p. 68.

TURRA, A. \& LEITE, F.P.P. 1995. Biologia populacional e reprodutiva e distribuição de três ermitões do gênero Clibanarius na região do Araçá, São Sebastião, SP. In X Mini-Simpósio de Biologia Marinha. São Sebastião, SP, p. 26-27.

TURRA, A. \& LEITE, F.P.P. 1996. Estratégias de ocupação de habitat por três espécies simpatricas de Clibanarius (Decapoda, Anomura, Diogenidae) na ilha de Pernambuco, Araçá, São Sebastião, SP. In III Simpósio sobre Oceanografia. São Paulo, SP, p. 231.

TURRA, A., DENADAI, M.R. \& LEITE, F.P.P. 1999. Períodos de atividade de quatro espécies simpátricas de ermitões no sudeste brasileiro. In XIV Simpósio de Biologia Marinha. São Sebastião, SP, p. 78.

TURRA, A., ARAÚJO, F.M.P., JACOBUCCI, G.B. \& LEITE, F.P.P. 1995. Os ermitões da Ilha de Pernambuco na Região do Araçá, São Sebastião, SP, Brasil. In X Mini-Simpósio de Biologia Marinha. São Sebastião, SP, p. 27.

VASCONCELOS, F.L. \& MANTELATTO, F.L.M. 2008. Distribuição espacial do caranguejo Panopeus occidentalis (Brachyura, Panopeidae), em manguezal remanescente do Litoral Norte de São Paulo. In V Congresso Brasileiro sobre Crustáceos. Sociedade Brasileira de Carcinologia, Porto Alegre, RS, p. 58.

VASCONCELOS, F.L., VERGAMINI, F.G. \& MANTELATTO, F.L.M. 2007. Crescimento relativo do caranguejo Panopeus occidentalis (Brachyura, Panopeidae). In Resumos do $15^{\circ}$ Simpósio Internacional de Iniciação Científica da USP. Ribeirão Preto, SP. (Zoologia - Resumo No 811).
VASCONCELOS, F.L., VERGAMINI, F.G. \& MANTELATTO, F.L.M. 2008. Aspectos populacionais do caranguejo Panopeus occidentalis (Brachyura, Panopeidae) em área de manguezal impactado do litoral norte de São Paulo. In XXVII Congresso Brasileiro de Zoologia. Curitiba, PR, p. 66.

VERGAMINI, F.G. \& MANTELATTO, F.L.M. 2005. Dados preliminares sobre a dinâmica populacional do caranguejo Panopeus americanus (Brachyura, Panopeidae) na região de São Sebastião, litoral norte paulista. In $13^{\circ}$ Simpósio Internacional de Iniciação Científica da USP. Ribeirão Preto, SP. (Zoologia - Resumo No 348 ).

VERGAMINI, F.G. \& MANTELATTO, F.L.M. 2006a. Avaliação populacional de um caranguejo panopeídeo (Decapoda, Brachyura) em uma área impactada do litoral norte paulista. In IV Congresso Brasileiro sobre Crustáceos. Guarapari, ES. (Ecologia - Resumo No 136).

VERGAMINI, F.G. \& MANTELATTO, F.L.M. 2006b. Caracterização populacional de Panopeus (Brachyura, Panopeidae) no litoral norte de São Paulo. In XXVI Congresso Brasileiro de Zoologia. Londrina, PR, p. 46.

VERGAMINI, F.G. \& MANTELATTO, F.L.M. 2006c. Período reprodutivo e recrutamento de juvenis do caranguejo Panopeus americanus (Crustacea, Brachyura, Panopeidae) na região do Araçá, litoral norte de São Paulo. In $14^{\circ}$ Simpósio Internacional de Iniciação Científica da USP. Ribeirão Preto, SP. (Zoologia - Resumo No 655).

WEHRTMANN, I., MIRANDA, I. \& MANTELATTO, F.L.M. 2007. Reproduction in the porcellanid crab Petrolisthes armatus (Decapoda: Anomura): Pacific versus Atlantic populations. In Program and Abstracts of the Crustacean Society Mid-Year Meeting. Coquimbo, Chile, p. 154.

WEHRTMANN, I.S., MIRANDA, I., BIAGI, R. \& MANTELATTO, F.L.M. 2006. A latitudinal comparison of the reproductive ecology of Petrolisthes armatus (Gibbes, 1850) (Anomura, Porcellanidae) in Pacific Costa Rica and Brazil. In IV Congresso Brasileiro sobre Crustáceos. Guarapari, ES, p. 63.

ZAGHA, K.A. \& VAL-SELLA, M.V. 1985. Caracterização das células prolactínica e gonodotrópicas do teleósteo marinho Bathygobius soporator. In XII Congresso Brasileiro de Zoologia. Campinas, SP, p. 205.

\section{Dissertações, teses e trabalhos de conclusão de curso}

ACEVEDO, A.L. 1974. Morfologia das brânquias e metabolismo respiratório de Aplysia brasiliana Rang, 1828 e Dendrodoris krebsii Morch, 1836 gastrópodes, opistobrânquios - Influência da luz. Dissertação de Mestrado, Universidade de São Paulo, São Paulo.

AGUJARO, L. 1996. Comportamento alimentar de Kalliapseudes schubarti Mae-Garzon, 1949 (Crustacea, Tanaidacea) na zona entremarés do Araçá, São Sebastião, SP. Monografia, Universidade Estadual de Campinas, Campinas.

ALVES, T.M. 2008. Contribuição ao conhecimento taxonômico de Terebellidae e Trichobranchidae (Annelida: Polychaeta) da região Sudeste-Sul do Brasil. Dissertação de Mestrado, Universidade de São Paulo, São Paulo.

ARANTES, I.C. 1994. Utilização e seleção de conchas por ermitões da zona entremarés na região do Araçá (SP). Dissertação de Mestrado, Universidade Estadual de Campinas, Campinas.

ARASAKI, E. 1997. Distribuição e estrutura da macrofauna bêntica no Canal de São Sebastião, São Paulo, Brasil. Dissertação de Mestrado, Universidade de São Paulo, São Paulo.

ARRUDA, E.P. 2000. Moluscos da região entremarés de praias arenosas do Litoral Norte do Estado de São Paulo. Dissertação de Mestrado, Universidade de São Paulo, São Paulo.

BARATA, G.F. 1996. Desenvolvimento gonadal e aspectos reprodutivos de três espécies de ermitões do gênero Clibanarius da região do Araçá, São Sebastião, SP. Monografia, Universidade Estadual de Campinas, Campinas.

BARROS, F.M.O. 2004. O comportamento de abandono de conchas por ermitões: fatores regulatórios em Pagurus criniticornis. Monografia de Conclusão de Curso, Centro Universitário da Fundação de Ensino Octávio Bastos, São João da Boa Vista. 
BELÚCIO, L.F. 1995. Comunidades bênticas de moluscos da zona entremarés da região do Araçá, São Sebastião, SP. Dissertação de Mestrado, Universidade Estadual de Campinas, Campinas.

BRASIL-LIMA, G.M.S.S. 1998. Efeitos de efluente doméstico do emissário submarino de São Sebastião (SP) sobre o fitoplâncton marinho: estudos de campo e laboratório. Tese de Doutorado, Universidade de São Paulo, São Paulo.

CARVALHO, E.P.E. 1999. Influência do sistema de disposição oceânica de São Sebastião - Praia do Araçá - em relação a baneabilidade das praias da margem continental do canal. Dissertação de Mestrado, Universidade Presbiteriana Mackenzie de São Paulo, São Paulo.

CARVALHO, M.S. 2003. Taxonomia de esponjas da Ordem Halichondrida (Demospongiae, Porifera) do Litoral Norte do Estado de São Paulo. Dissertação de Mestrado, Universidade Federal do Rio de Janeiro, Rio de Janeiro.

CHRISTOFFERSEN, M.L. 1980. Taxonomia e distribuição dos Alpheoidea (Crustacea, Decapoda, Natantia) do Brasil, Uruguai e norte da Argentina, incluindo considerações sobre a divisão do sul do continente em províncias biogeográficas marinhas. Tese de doutorado, Universidade de São Paulo, São Paulo.

CUNHA, I. 1996. Sustentabilidade e poder local: a experiência de política ambiental em São Sebastião, costa norte do Estado de São Paulo (19891992). Tese de doutorado, Universidade de São Paulo, São Paulo.

DITADI, A.S.F. 1969a. Contribuição ao estudo da ecologia e fisiologia de Lissomyema exilli (F. Müller, 1883) (Echiura). Tese de Doutorado, Universidade de São Paulo, São Paulo.

DOLDER, M.A.H. 1973. Ultraestrutura e citoquímica das junções neuromusculares em Equinodermes: Asterina stellifera Moebius, 1859 (Asteroidea) e Pentacta peterseni Acona Lopez, 1965 (Holothuroidea). Tese de Doutorado, Universidade de São Paulo, São Paulo.

DUARTE, L.F.L. 1980b. A Endofauna da esponja Zygomycale parishii (Bowerbank) (composição, dominância, diversidade e natureza da associação). Dissertação de Mestrado, Universidade Estadual de Campinas, Campinas.

FARIA, M.T.C. 1974. Fisioecologia de Eunice sebastiani (Anelídeo Poliqueto - Eunicídeo) - neurosecreção e osmoregulação. Dissertação de Mestrado, Universidade de São Paulo, São Paulo.

FRANCISCO, J. 2002b. Desconstrução do lugar - o aterro da Praia da Frente do centro histórico de São Sebastião (SP). Tese de Doutorado, Universidade Estadual Paulista Júlio de Mesquita Filho, Rio Claro.

GARCIA, A.L.B. 1979. Desenvolvimento pós-embrionário de Clibanarius antillensis Stimpson, 1859 e Pagurus criniticornis (Dana, 1852) (Crustacea, Paguridea). Dissertação de Mestrado, Universidade Estadual Paulista Júlio de Mesquita Filho, Rio Claro.

GARCIA, L.T. 2007. Determinação de hidrocarbonetos aromáticos policíclicos (HAPs) nos sedimentos da região circunjacente aos emissários submarinos de esgoto do TEBAR e Araçá, São Sebastião - SP. Iniciação Científica, Centro Universitário Fundação Instituto de Ensino para Osasco, Osasco.

GARCIA, R.B. 2006. Muda e crescimento em ermitões (Crustacea, Diogenidae): os efeitos da presença dos conspecíficos e a influência da concha. Pós-Doutorado, Universidade de São Paulo, São Paulo.

GENOFRE NETO, G.C. 1972. Mudança de cor em Uca rapax (Smith) Crustaceo Decapode: estudo da cromatóforotropina. Dissertação de Mestrado, Universidade de São Paulo, São Paulo.

GOMES-CORRÊA, M.M. 1986. Stomatopoda do Brasil (Crustacea Hoplocarida). Tese de Doutorado, Universidade de São Paulo, São Paulo.

GORNI, R. 1999. Ocorrência e distribuição de pesticidas organoclorados $(\mathrm{OCs})$ e bifenilos policlorados (PCBs) na região da plataforma interna do Canal de São Sebastião. Dissertação de Mestrado, Universidade de São Paulo, São Paulo.

GUBITOSO, S. 2006. Estudo geoambiental do entorno do emissário submarino do Araçá, São Sebastião, SP. Trabalho de Conclusão de Curso, Universidade de São Paulo, São Paulo.
IGUCHI, J.M.S. 2008. Investimento reprodutivo do caranguejo Panopeus americanus em uma área de manguezal impactada (São Sebastião, São Paulo). Monografia de Bacharelado, Universidade de São Paulo, Ribeirão Preto.

JACOBI, C.B. 1992. Efeitos do naftaleno na sobrevivência, desenvolvimento e metabolismo respiratório de larvas de crustáceos anomuros. Tese de doutorado, Universidade de São Paulo, São Paulo.

JACOBUCCI, G.B. 1996. Padrões de dispersão e agrupamento em ermitões (Decapoda, Anomura) na região do Araçá, São Sebastião,SP. Iniciação Científica, Instituto de Biologia, Universidade Estadual de Campinas.

KATTAR, M.R. 1966. Estudo dos protozoários ciliados Psamófilos. Tese de Doutorado, Universidade de São Paulo, Ribeirão Preto.

KAWAKAMI, S.K. 1999. Coprostanol como um indicador químico de contaminação por esgoto urbano em algumas regiões do litoral de São Paulo. Dissertação de Mestrado, Universidade de São Paulo, São Paulo.

KAWAUCHI, G.Y. 2005. Sipuncula do litoral Sul e Sudeste do Brasil. Tese de Doutorado, Universidade de São Paulo, São Paulo.

LACERDA, T.P. 1981. Variação da glicose na hemolinfa de Callinectes danae Smith, 1869 (Crustacea - Decapoda - Portunidae). Tese de Doutorado, Universidade de São Paulo, São Paulo.

LEAHY, W.M. 1970. Fisioecologia e osmoregulação de Aplysia brasiliana (Rang, 1828) - Molusco Gastrópode Opistobrânquio Tectibrânquio. Dissertação de Mestrado, Universidade de São Paulo, São Paulo.

LEAHY, W.M. 1975. Respostas dos músculos do esôfago e do coração de Aplysia brasiliana (Molusco - Gastrópode) à Acetilcolina e à 5-Hidroxitriptamina, antes e após supressão de íons cálcio e magnésio da solução perfusora. Tese de Doutorado, Universidade de São Paulo, São Paulo.

LEONEL, R.M.V. 1981. Influência da salinidade sobre algumas respostas osmóticas de Anomalocardia brasiliana (Gmelin, 1791) (Mollusca: Bivalvia). Tese de Doutorado, Universidade de São Paulo, São Paulo.

LIMA, G.M.S.S.B. 1998. Efeitos do efluente doméstico do emissário submarino de São Sebastião (SP), sobre o fitoplâncton marinho: estudos de campo e laboratório. Tese de Doutorado, Universidade de São Paulo, São Paulo.

LIMA, H.H. 1972. Fisioecologia de anelídeos poliquetos - Osmorregulação. Tese de Doutorado, Universidade de São Paulo, São Paulo.

LOPES, P.P. 1993. Estrutura da comunidade de poliquetos da zona entremarés da região do Araçá, São Sebastião (SP). Dissertação de Mestrado, Universidade Estadual de Campinas, Campinas.

MAGALHÃES, C.A. 1988. Padrões de variação morfológica em Thais haemastoma L., 1767 (Gastropoda: Prosobranchia). Dissertação de Mestrado, Universidade Estadual de Campinas, Campinas.

MARCELLINO, E.B. 2000. Sistematização dos projetos de emissários submarinos da SABESP e avaliação de desempenho através do modelo computacional CORMIX. Dissertação de Mestrado, Universidade de São Paulo, São Paulo.

MARQUES, A.C. 1993. Sistemática de Eudendriidae L. Agassiz, 1862 (Cnidaria, Hydrozoa) do litoral paulista. Dissertação de Mestrado, Universidade de São Paulo, São Paulo.

MARQUES, S.M. 1972. Contribuição para o conhecimento da Etologia de Uca rapax - Crustacea, Decapoda, Brachiura - no ambiente natural e em condições experimentais. Dissertação de Mestrado, Universidade de São Paulo, São Paulo.

MENDES, F.M. 2007. Estrutura da comunidade macrozoobentônica na região de influência do Terminal Almirante Barroso (TEBAR) no Canal de São Sebastião (SP, Brasil). Dissertação de Mestrado, Universidade de São Paulo, São Paulo.

MIGOTTO, A.E. 1993. Hidróides (Hydrozoa, Cnidaria) marinhos bentônicos da região costeira de São Sebastião, SP. Tese de Doutorado, Universidade de São Paulo, São Paulo.

MILANELLI, J.C.C. 2003. Biomonitoramento de costões rochosos. Instrumento para avaliação de impactos gerados por vazamento de óleo na região do Canal de São Sebastião - São Paulo. Tese de Doutorado, Universidade de São Paulo, São Paulo. 
MORGADO, E.H. 1980b. A endofauna de Schizoporella unicornis (Johnston, 1874) (Bryozoa), no Litoral Norte do Estado de São Paulo. Dissertação de Mestrado, Universidade Estadual de Campinas, Campinas.

MOSSOLIN, E.C. 1994. Efeito, a curto prazo, da ablação do pedúnculo ocular no metabolismo respiratório de Callinectes danae Smith 1869 (Crustacea, Decapoda, Portunidae). Monografia de Bacharelado, Universidade de Santo Amaro, Santo Amaro.

NETTO, L.F. 2006. Echinodermata do canal de São Sebastião (SP). Dissertação de Mestrado, Universidade de São Paulo, São Paulo.

NUCCI, P.R. 1998. Biodiversidade de crustáceos da região entremarés em praias do Canal de São Sebastião (SP). Dissertação de Mestrado, Universidade Estadual Paulista Júlio de Mesquita Filho, Rio Claro.

PADOVANNI, N.O. 2009. Macrofauna e ecologia de uma praia areno-lamosa do município de São Sebastião, SP. Monografia de Conclusão de Curso, Universidade Estadual de Londrina, Paraná.

PETERSEN, J.A. 1965. Contribuição para o conhecimento da ecologia e da fisiologia de Enteropneustos do Brasil com descrição de uma nova espécie Willeyia loya sp. n.. Tese de Doutorado, Universidade de São Paulo, São Paulo.

PLASTINO, E.M. 1991. Cultivo in vitro, estudos reprodutivos e biossistemática de algas gracilarióides (Rhodophyta, Gracilariales) de talo cilíndrico. Tese de Doutorado, Universidade de São Paulo, São Paulo.

PRADO, A. 1999. Taxonomia, distribuição e ecologia da família Grapsidae (Crustacea, Decapoda, Brachyura) no litoral brasileiro. Dissertação de Mestrado, Universidade Estadual Paulista Júlio de Mesquita Filho, Rio Claro.

RAMOS, M.P. 1994. Aspectos da reprodução de Kalliapseudes schubarti Mañe-Garzon, 1949 (Crustacea, Tanaidacea) da região do Araçá, São Sebastião, SP. Trabalho de Conclusão de Curso, Pontifícia Universidade Católica de Campinas, Campinas.

RODRIGUES, S.A. 1966 Estudos sobre Callianassa. Tese de Doutorado, Universidade de São Paulo, São Paulo.

RODRIGUES, S.A. 1983 Aspectos da biologia de Thalassinidea do Atlântico tropical americano. Tese de Livre Docência, Universidade de São Paulo, São Paulo.

ROSA, L.L.L. 1973. Fisioecologia do Hemicordado Balanoglossus gigas (Spengel, 1893). Estrutura do epitélio tegumentário e comportamento. Dissertação de Mestrado, Universidade de São Paulo, São Paulo.

ROSSI, M.C.S. 2008. Contribuição ao conhecimento taxonômico dos poliquetas sabelídeos (Polychaeta: Sabellidae) da região Sudeste do Brasil. Dissertação de Mestrado, Universidade de São Paulo, São Paulo.

SILVA, I.M. 2007. Ecologia de Petrolisthes armatus (Gibbes, 1850) (Crustacea, Anomura, Porcellanidae) na região do Araçá, São Sebastião, São Paulo. Dissertação de Mestrado, Universidade de São Paulo, Ribeirão Preto.

SOARES, A.M.V. 1970. Sobre a biologia de Renilla sp. (Coelenterata Anthozoa), estudo da bioluminescência. Dissertação de Mestrado, Universidade de São Paulo, São Paulo.

SOTO-ESPINOSA, D. 1993. Biomassa e estrutura populacional de Kalliapseudes schubarti Mañe-Garzon, 1949 (Crustacea, Tanaidacea) na zona entremarés do Araçá, São Sebastião, SP. Monografia, Universidade Estadual de Campinas, Campinas.

SOUZA, E.C.F. 2003. Atração de Pagurus criniticornis Dana, 1852 (Decapoda, Anomura, Paguridae) para eventos de predação simulada. Dissertação de Mestrado, Universidade Estadual de Campinas, Campinas.

STEINER, T.M. 2000. Eunicida e Phyllodocida (Polychaeta, Aciculata) de praias do litoral norte do Estado de São Paulo. Dissertação de Mestrado, Universidade de São Paulo, São Paulo.

TURRA, A. 1998. Estratégias de vida de três espécies simpátricas de ermitões do gênero Clibanarius (Decapoda, Anomura, Diogenidae) na região entremarés da Ilha de Pernambuco, São Sebastião, SP, Brasil. Dissertação de Mestrado, Universidade Estadual de Campinas, Campinas.

TURRA, A. 2003. Crescimento, seleção de conchas, predação e reprodução dos ermitões da região do Araçá, São Sebastião, SP. Tese de Doutorado, Universidade Estadual de Campinas, Campinas.
VANI, Y.S. 1987. Estudos com o sistema bioluminescente do verme anelídeo Chaetopterus variopedatus. Dissertação de Mestrado, Universidade de São Paulo, São Paulo.

VASCONCELOS, F.L. 2008. Biologia populacional do caranguejo Panopeus occidentalis Saussure, 1857 (Decapoda, Brachyura, Panopeidae) na região do Araçá, São Sebastião, litoral norte de São Paulo. Monografia de Bacharelado, Universidade de São Paulo, Ribeirão Preto.

VERGAMINI, F.G. 2006. Dinâmica populacional do caranguejo Panopeus americanus Saussure, 1857 (Decapoda, Brachyura, Panopeidae) na região do Araçá, São Sebastião, litoral norte de São Paulo. Trabalho de Conclusão de Curso, Universidade de São Paulo, Ribeirão Preto.

YOUNG, P.S. 1987. Taxonomia e distribuição da subclasse Cirripedia no Atlântico sul ocidental. Tese de Doutorado, Universidade de São Paulo, São Paulo.

ZANARDI, E. 1996. Hidrocarbonetos no canal de São Sebastião e na plataforma interna adjacente - influencia do derrame de maio de 1994. Dissertação de Mestrado, Universidade de São Paulo, São Paulo.

\section{Dissertações e teses em andamento}

CORTE, G.N. Biologia populacional e reprodutiva de Anomalocardia brasiliana (Gmelin, 1791) (Bivalvia: Veneridae). Dissertação de Mestrado, Universidade Estadual de Campinas, Campinas. (Início: março/2008 e termino previsto: julho/2010. Orientador: A. Cecília Z. Amaral. Bolsa FAPESP).

FUKUDA, M.V. Contribuição ao conhecimento dos poliquetas silídeos (Polychaeta: Syllidae) da região Sudeste-Sul do Brasil. Tese de Doutorado, Universidade de São Paulo, São Paulo. (Início: 2005 e término previsto: 2009. Orientador: João M.M. Nogueira. Bolsa FAPESP).

QUAST, M.P. Investigação de limites específicos em Corbula (Corbulidae: Bivalvia) do Brasil, com base em marcadores moleculares. Tese de Doutorado, Universidade Estadual de Campinas, Campinas. (Início: agosto/2007 e término previsto: julho/2011. Orientador: A. Cecília Z. Amaral. Bolsa FAPESP).

YOKOYAMA, L.Q. Dieta alimentar, alometria, longevidade e reprodução de Olivella minuta (Link, 1807) (Gastropoda, Olividae). Pós-Doutorado, Universidade de São Paulo, São Paulo. (Início: janeiro/2010 e término previsto: dezembro/2012. Supervisor: Alexander Turra).

PETRACCO, M. Produção secundária da macrofauna bentônica de praias arenosas: estudo de caso com Olivella minuta (Link, 1807) (Mollusca, Gastropoda) e análise e validação métodológica. Pós-Doutorado, Universidade de São Paulo, São Paulo. (Início: janeiro/2010 e término previsto: dezembro/2012. Supervisor: Alexander Turra).

\section{Texto publicado em meio digital}

AMARAL, A.C.Z., NALLIN, S.A.H. \& STEINER, T.M. 2006. Catálogo das espécies de Annelida Polychaeta do Brasil. http://www.ib.unicamp.br/ projbiota/bentos_marinho/catalogo_polychaeta2006.pdf (último acesso em 25/05/2009)

\section{Relatório técnico}

CEBIMar - Centro de Biologia Marinha da USP. 1987. Parecer sobre o RIMA referente ao aterro da praia do Araçá, São Sebastião. USP, São Sebastião.

PRONABIO. 1999. Relatório de sistematização de informação para os Estados do Espírito Santo, Rio de Janeiro, São Paulo e Paraná. PRONOBIO, Brasília, DF.

\section{Artigos de jornal}

AQUINO, A.C. 2008. Plano de Desenvolvimento do Porto de São Sebastião prevê aterro do Mangue do Araçá e revolta ambientalistas. Jornal Imprensa Livre, 8 de março de 2008.

BUSSINGER, F. 2008. O Porto de São Sebastião e o meio ambiente. Coluna Opinião Livre, Jornal Imprensa Livre, 15 de março de 2008. http://www. imprensalivre.com/articulistas/index.php?idart=134\&idartigo $=1039$ (último acesso em 25/05/2009) 
BUSSINGER, F. 2008. O Porto de São Sebastião e o meio ambiente. Informe do Porto de São Sebastião, Coluna Opinião Livre, Jornal Imprensa Livre, 15 de março de 2008. http://www.portodesaosebastiao.com.br/ arquivo/0005.shtml (último acesso em 25/05/2009)

BRANDALISE, V.H. \& ALMEIDA, R. 2008. Expansão do Porto vai aterrar mangue em SP. Jornal o Estado de São Paulo, Vida, 11 de outubro de 2008, p. A31. http://pib.socioambiental.org/en/noticias?id=61288 (último acesso em 25/05/2009)

CEDS - LITORAL NORTE. 2009. Debate avalia impactos ambientais do plano de expansão do Porto de São Sebastião. Notícias, Centro de Experimentação em Desenvolvimento Sustentável. http://www.cedslitoralnorte.org.br/ver_noticia. php?id=15\&pag=1 (último acesso em 25/05/2009)

COSTA, A.C.P. 2008. O porto de São Sebastião. Jornal o Estado de São Paulo, março de 2008.

CREDENDIO, J.E. Para ampliar porto, Estado quer aterrar manguezal. Folha de São Paulo, Caderno Cotidiano, 12 de março de 2008.

GIL, S. 2008. Estado altera APA e Greenpeace anuncia apoio ao projeto. Jornal Imprensa Livre, 28 de maio de 2008. http://www.imprensalivre. com/busca/top_busca.php?edit=3\&id=24858 (último acesso em 25/05/2009)

HIPÓLITO, E. 2008. Lá vem as APAs outra vez! São Sebá em Opinião, 23/06/2008. http://saoseba.zip.net/arch2008-06-01_2008-06-30.html (último acesso em 25/05/2009)

MIGOTTO, A.E. 2008. O aterro do mangue do Araçá eliminará um dos mais peculiares ambientes marinhos do litoral. O Estado de São Paulo, 16 de março de 2008.

MORAES, C. 2008. Prefeitura municipal apresenta estudo para terminal de navios de passageiros à autoridades federais. Jornal Imprensa Livre, 21 de abril de 2008. http://www.imprensalivre.com/busca/top_busca. php?edit=2\&id=5740 (último acesso em 25/05/2009)

PIRES, F. 2008. Expansão do porto ocorrerá em etapas. A Tribuna Digital, 18 de março de 2008.

RAMOS, R.H.P. 2008. São Sebastião-SP - “Socorro!”. AMARRIBO - Amigos Associados de Ribeirão Bonito. http://www.amarribo.org.br/mambo/ index.php?option $=$ com_content $\&$ task $=v i e w \& i d=2019 \&$ Itemid $=68$ (último acesso em 25/05/2009)

REDAÇÃO DO JORNAL DA ORLA. 2008. O porto de São Sebastião vem aí. Jornal da Orla, 22 de abril de 2008. http://www.jornaldaorla.com.br/ noticias_integra.asp?cd_noticia=2180 (último acesso em 25/05/2009)

REDAÇÃO DO JORNAL DA ORLA. 2008. O porto de São Sebastião vem aí. Informe do Porto de São Sebastião, 27 de abril de 2008. http://www. portodesaosebastiao.com.br/arquivo/0010.shtml (último acesso em $25 / 05 / 2009)$

REGO, E.H. 2008. Ampliar o porto sem destruir: será só um chavão? Jornal Canal Aberto, 14 de abril de 2008. http://www.jornalcanalaberto.com.br/ jca.php?pagina $=$ materias\&cod_editoria $=61 \&$ cod_materia $=4072$ (último acesso em 25/05/2009)

REIS, V. 2008. O Estado e o Meio Ambiente. O Ambiente Inteiro, 28 de setembro de 2008.http://oambienteinteiro.blogspot.com/2008/09/oestado-e-o-meio-ambiente.html (último acesso em 25/05/2009)

ROCHA, B. 2008. Aterro do Mangue do Araçá São Sebastião, SP. Blog Ambiental, 17 de abril de 2008. http://jornalecologico.blogspot. com/2008/04/aterro-do-mangue-do-ara-so-sebastio-sp.html (último acesso em 25/05/2009)

RODRIGUES, C. 2008. Planos de ampliação do porto serão debatidos no Plano Diretor. Jornal Imprensa Livre, 5 de janeiro de 2008.

RODRIGUES, C. 2008. Secretaria dos Transportes levará novo traçado do trecho de serra da Tamoios ao governador no início de maio. Jornal Imprensa Livre, 23 de abril de 2008.

SALGADO, R. 2009. Debate sobre ampliação do porto discute preservação do Mangue do Araçá e novo acesso rodoviário. Jornal Imprensa Livre, 14 de fevereiro de 2009. http://www.imprensalivre.com/busca/top_busca. php?edit=2\&id=6352 (último acesso em 25/05/2009)
SALGADO, R. 2009. Mais restritiva, Lei do Gerenciamento Costeiro não é aplicada por prefeituras. Jornal Imprensa Livre, 04 de maio de 2009. http://www.imprensalivre.com/busca/top_busca.php?edit=3\&id=28579 (último acesso em 25/05/2009)

SIMÕES, N. 2008. A história do porto de São Sebastião e o novo plano de ampliação. Jornal Canal Aberto, 11 de abril de 2008. http://www.jornalcanalaberto.com.br/jca.php?pagina=materias\&cod editoria=61\&cod_materia=4077 (último acesso em 25/05/2009)

SIMÕES, N. 2008. Ante-projeto de ampliação do porto de São Sebastião prevê a construção de pier para atracação de 10 navios. Jornal Canal Aberto, 12 de abril de 2008. http://www.jornalcanalaberto.com.br/jca. php?pagina $=$ materias \&cod_editoria $=61 \& \operatorname{cod} \_$materia $=4100$ (último acesso em 25/05/2009)

SIMÕES, N. \& SIQUEIRA, F.A. 2008. A arapuca do Araçá. Jornal Canal Aberto, 11 de abril de 2008. http://www.jornalcanalaberto.com.br/jca. php?pagina $=$ materias $\&$ cod_editoria $=61 \& \operatorname{cod} \_$materia $=4080$ (último acesso em 25/05/2009)

SIMÕES, N. \& SIQUEIRA, F.A. 2008. Uma pequena expedição fotográfica pela baía do Araçá. Jornal Canal Aberto, 14 de abril de 2008. http:// www.jornalcanalaberto.com.br/jca.php?pagina $=$ materias\&cod_

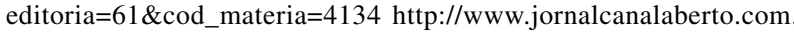
$\mathrm{br} /$ index.php?codgrupo=10\&pagina $=$ galeriafoto (último acesso em 25/05/2009)

SIMÕES, N. \& SIQUEIRA, F.A. 2008. Porto de São Sebastião e Petrobrás planejam construir píer no mesmo local. Jornal Canal Aberto, 18 de abril de 2008. http://www.jornalcanalaberto.com.br/jca. php?pagina $=$ materias $\&$ cod_editoria $=61 \& \operatorname{cod} \_$materia $=4290$ (último acesso em 25/05/2009)

SIMÕES, N. \& SIQUEIRA, F.A. 2008. Muito além das parelhas. Editorial do Jornal Canal Aberto, 02 de maio de 2008. http:// www.jornalcanalaberto.com.br/index.php?pagina $=$ materias\&cod editoria=32\&cod_materia=4637 (último acesso em 25/05/2009)

SIMÕES, N. \& SIQUEIRA, F.A. 2009. Ampliação do Porto é debatida na Câmara de São Sebastião. Jornal Canal Aberto, 20 de fevereiro de 2009. http://www.jornalcanalaberto.com.br/index.php?pagina=materias\&cod editoria $=3 \&$ cod_materia $=10491 \&$ palavrachave $=$ amplia $\%$ C3\%A7\%C3\% A3o\&inicio $=0$ (último acesso em 25/05/2009)

TEIXEIRA, L. 2007. Emissário submarino do Araçá completa 20 anos de existência sem licenciamento ambiental. Jornal Imprensa Livre, 26 de dezembro de 2007.

TEIXEIRA, L. 2008. Duplicação da Tamoios só será prioridade quando o Porto de São Sebastião for ampliado. Jornal Imprensa Livre, 13 de março de 2008.

TEIXEIRA, L. 2008. É mangue ou não é? Jornal Imprensa Livre, 01 de agosto de 2008.

TEIXEIRA, L. 2008. Estado quer compatibilizar uso sustentável com conservação da natureza transformando as ilhas marinhas em Área de Proteção Ambiental. Jornal Imprensa Livre, 25 de abril de 2008.

TEIXEIRA, L. 2008. I reunião Plenária do CBH-LN de 2008 traz novidades sobre o plano de desenvolvimento do Porto de São Sebastião. Jornal Imprensa Livre, 7 de março de 2008.

TEIXEIRA, L. 2008. Mangue do Araçá ganha projeto de recuperação vinculado ao Parque de Valorização de Resíduos Urbanos. Jornal Imprensa Livre, 02 de agosto de 2008. http://www.imprensalivre.com.br/busca/ top_busca.php?edit=3\&id=25541 (último acesso em 25/05/2009)

TEIXEIRA, L. 2008. Xico Graziano assina projeto de dragagem do Rio Una e Cetesb promete licença do emissário submarino do Araçá parada há 20 anos. Jornal Imprensa Livre, 05 de junho de 2008. http://www.imprensalivre.com/busca/top_busca.php?edit=2\&id=5811 (consultado em 25/09/2009)

VERÍSSIMO, M. 2008. Proposta de aterro ameaça ecossistema: governo estadual quer instalar área retroportuária na baía do Araçá, em São Sebastião. Jornal Vale Paraibano, 22 de março de 2008.

PORTOGENTE 2009. Camada pré-sal mudará rotina do Porto de São Sebastião. Portos do Brasil - São Sebastião, 24 de fevereiro de 2009. http://www. 
portogente.com.br/portosdobrasil/texto.php?cod=7\&txt=3732 (último acesso em 30/09/2009)

PORTOGENTE 2009. Estado de São Paulo concentra 93\% das cargas nas rodovias. Portos do Brasil - São Sebastião, 24 de fevereiro de 2009. http:// www.portogente.com.br/texto.php?cod=21575\&sec=10 (último acesso em 30/09/2009)

PORTOGENTE 2009. Expansão polêmica. Portos do Brasil - São Sebastião, 25 de agosto de 2009. http://www.portogente.com.br/texto. php?cod=24600\&sec=5 (último acesso em 30/09/2009)

PORTOGENTE 2009. Projeto do governo Serra pode gerar caos, alerta ambientalista. Portos do Brasil - São Sebastião, 22 de setembro de 2009. http://www.portogente.com.br/portosdobrasil/texto.php?cod=7\&txt=3772 (último acesso em 30/09/2009)

PORTOGENTE 2009. Expansão do Porto de São Sebastião trará mais prejuízos do que benefícios, alerta consultor. Portos do Brasil - São Sebastião, 29 de setembro de 2009. http://www.portogente.com.br/portosdobrasil/texto. php?cod=7\&txt=3775 (último acesso em 30/09/2009)

\section{Artigos de jornal publicados em meio digital}

Audiência aprova plano de expansão portuária. 2009. Jornal A Tribuna, 17 de fevereiro de 2009. http://www.sindaport.com.br/not.asp?seq=269 (último acesso em 25/05/2009)

Audiência aprova plano de expansão portuária. 2009. Jornal A Tribuna, 17 de fevereiro de 2009. http://www.webtranspo.com.br/modais/ aquaviario/2194-audiencia-aprova-plano-de-expansao-do-porto-de-saosebastiao.html (último acesso em 25/05/2009)
Comitê aponta divergência entre Petrobras e Porto na construção dos píeres sobre o Araçá. 2008. Jornal Imprensa Livre, 12 de setembro de 2008 http://www.imprensalivre.com.br/busca/top_busca. php?edit=3\&id=25926 (último acesso em 25/05/2009)

Mangue do Araçá ganha projeto de recuperação vinculado ao Parque de Valorização de Resíduos Urbanos. 2008. Litoral Virtual, 04 de agosto de 2008. http://litoralvirtual.com.br/noticias/2008/08/04.html\#SaoSebastiao (último acesso em 25/05/2009)

Para ampliar porto, Estado quer aterrar manguezal. 2008. Jornal Folha de São Paulo, 12 de março de 2008. 12 de março de 2008. http://www.intelog.com.br/site/ default.asp?TroncoID=907492\&SecaoID $=508074 \&$ SubsecaoID $=948063 \&$ Template=../artigosnoticias/user_exibir.asp\&ID=940817\&Titulo=Para\%20 ampliar\%20porto\%2C\%20Estado\%20quer\%20aterrar\%20manguezal (último acesso em 25/05/2009)

Para ampliar porto, Serra quer aterrar manguezal. 2008. Olhando o Movimento, 12/03/2008. http://olhandoosmovimentos.blogspot.com/2008/03/paraampliar-porto-serra-quer-aterrar.html (último acesso em 13/05/2009)

Proposta gera polêmica. 2008. A Tribuna Digital, 18 de março de 2008.

Xico Graziano divulga nota sobre criação de mosaico de ilhas e áreas de preservação ambiental marinhas. 2008. Jornal Canal Aberto, 12 de maio de 2008. http://www.jornalcanalaberto.com.br/index. php?pagina $=$ materias $\&$ cod_editoria $=28 \&$ cod_materia $=4877$ (consultado em 25/05/2009)

O Porto de São Sebastião e o meio ambiente. 2008. Porto de São Sebastião, 15/03/2008. http://www.portodesaosebastiao.com.br/arquivo/0005.shtml (último acesso em 13/05/2009) 